Portland State University

PDXScholar

Summer 7-21-2016

\title{
Expanding the Versatility of Nano Assembled Capsules as Platform of Potential High Payload MRI Contrast Agents
}

Annah Farashishiko

Portland State University

Follow this and additional works at: https://pdxscholar.library.pdx.edu/open_access_etds

Part of the Chemistry Commons, and the Nanoscience and Nanotechnology Commons Let us know how access to this document benefits you.

\section{Recommended Citation}

Farashishiko, Annah, "Expanding the Versatility of Nano Assembled Capsules as Platform of Potential High Payload MRI Contrast Agents" (2016). Dissertations and Theses. Paper 3085.

https://doi.org/10.15760/etd.3080

This Dissertation is brought to you for free and open access. It has been accepted for inclusion in Dissertations and Theses by an authorized administrator of PDXScholar. Please contact us if we can make this document more accessible: pdxscholar@pdx.edu. 
Expanding the Versatility of Nano Assembled Capsules as Platform of Potential High

Payload MRI Contrast Agents

by

Annah Farashishiko

A dissertation submitted in partial fulfillment of the requirements for the degree of

Doctor of Philosophy

in

Chemistry

\section{Dissertation Committee: \\ Mark Woods, Chair \\ Andrea Goforth \\ Robert Strongin \\ Erik Johansson \\ Jason Podrabsky}

Portland State University

2016 


\begin{abstract}
Magnetic resonance imaging (MRI) has become a powerful clinical modality in
\end{abstract} diagnostic medicine. It is non-invasive and offers high spatial and temporal resolution. The goal of molecular imaging is to reveal the pathophysiology underlying the observed anatomy and diagnose diseases. The detection of pathological biomarkers can lead to early recognition of diseases and improved monitoring for recurrence. Clinically available contrast agents are limited in their discrimination of contrast between tissues and they tend to have very high detection limits. Because biomarkers are very low in concentration there is a need for high payload deposition of contrast agent (CA) and targeted imaging. Encapsulating discrete $\mathrm{Gd}^{3+}$ chelates in nano assembled capsules (NACs) is a simple and effective method of preparing an MRI contrast agent capable of delivering a large payload of high relaxivity imaging agent. The preparation of contrast agent containing NACs had previously focused on preparations incorporating GdDOTP ${ }^{5-}$ into the internal aggregate. In this report we demonstrate that other $\mathrm{Gd}^{3+}$ chelates bearing overall charges as low as 2- can also be used to prepare NACs. This discovery opens up the possibility of using $\mathrm{Gd}^{3+}$ chelates that have inner-sphere water molecules that could further increase the relaxivity enhancement associated with the long rotational correlation time $\left(\tau_{\mathrm{R}}\right)$ that arises from encapsulation. However, encapsulation of the $q=1$ chelate GdDTPA $^{2-}$ afforded the same increase in relaxivity as the outer-sphere chelate GdTTHA $^{3-}$. This leads us to the conclusion that in the NAC interior proton transport is not mediated by movement of whole water molecules and the enhanced relaxivity of $\mathrm{Gd}^{3+}$ chelate encapsulated within NACs arises primarily from second sphere effects. The 
nano assembled capsule platform has been further expanded by an alternative coating method, a new cross linked peptidic shell reported in this work affords robust capsules and exceptionally high per $\mathrm{Gd}^{3+}$ relaxivities $\left(70.7 \mathrm{mM}^{-1} \mathrm{~s}^{-1}\right)$. The availability of free amines on the surface of these capsules can be exploited to attach targeting moieties. This was demonstrated through the reaction of fluorescein isothiocyanate (FITC), an intense green emitting dye, with these amines. Green emission from the capsules indicated that surface amines were accessible to FITC. Unlike $T_{1}$-shortening contrast agents, paraCEST agents can be switched on and off by the imaging scientist by turning on and off a pre-saturation pulse. This affords the ability to acquire both pre- and postcontrast images even after administration of a paraCEST contrast agent. This could potentially eliminate problems co-registering pre- and post-contrast images. A reverse NAC may allow a cationic paraCEST contrast agent to be incorporated in a high payload NAC. We were successful in synthesizing a reverse capsule using DyDOTAM $^{3+}$, a paraCEST agent, and the negatively charged polymer polyacrylate and encapsulated with $\mathrm{SiO}_{2}$ nanoparticles. These initial preparations of reverse NACs were not able to generate CEST contrast however. 


\section{Dedication}

This is for you mama and papa, my siblings Lucy, Tendai and Simon thank you guys for all the support and I love you.

As for cancer I beat you on this. 


\section{Acknowledgements}

I would like to thank my research advisor Dr Mark Woods, for all the support and assistance he gave me throughout this programee, and just believing in me that I can do it. Thank you so much for being flexible with my health, I am out of words all I can say is thank you so much. I did learn a lot from you.

To the Woods research group, I owe you big time, thank you so much for all the support both academically and socially. I feel like we were now one big family. Thank you for all the support you gave me when my health was on the down side (Dr Benjamin Webber, Dr Julian Haig, Katherine Payne, Jacqueline Slack, Lauren Rust and Karley Maier, Joseph Armstrong). Words only cannot express how much I appreciate all your support.

My research committee Dr Andrea Goforth, Robert Strongin, Erik Johansson and Jason Podrabsky for your time and being patient with me all the way.

Dr Andrea Goforth thank you so much for allowing me to use the DLS and tolerating me when I was using the DLS, running my never ending measurements

Dr Jason Podrabsky, I really appreciate you for allowing me to use your confocal microscope thanks. 
Table of Contents

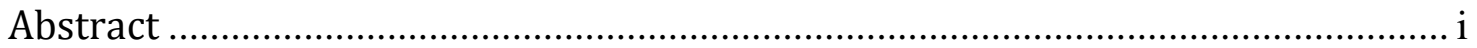

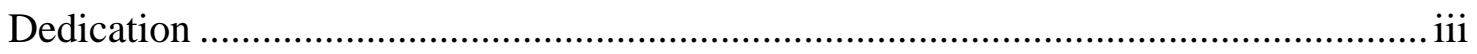

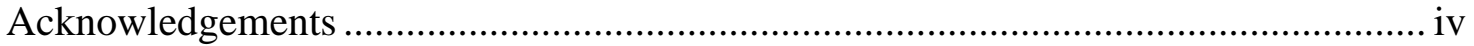

List of Tables ................................................................................................. viii

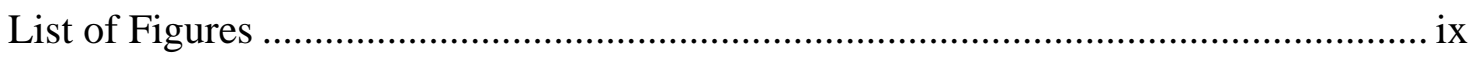

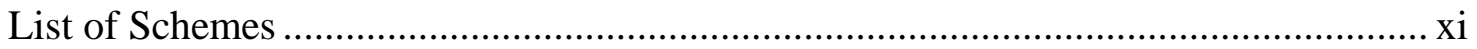

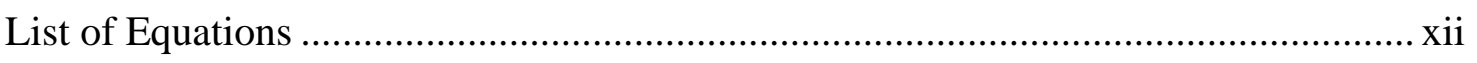

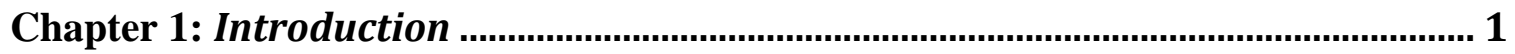

1.1 A look back at where we have been and where we are ..................................... 1

1.2 Basic principle of Magnetic Resonance ............................................................ 4

1.2.1: Inversion recovery pulse sequence............................................................ 7

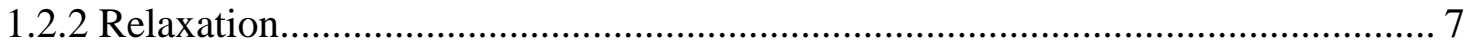

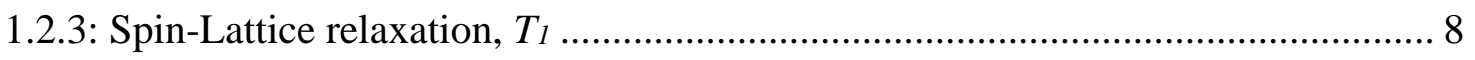

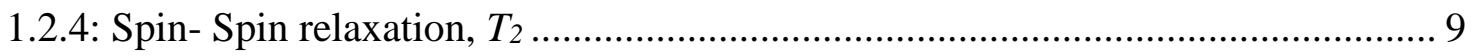

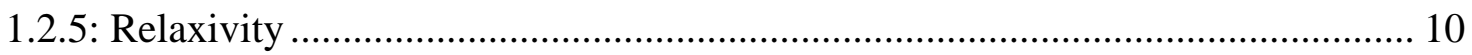

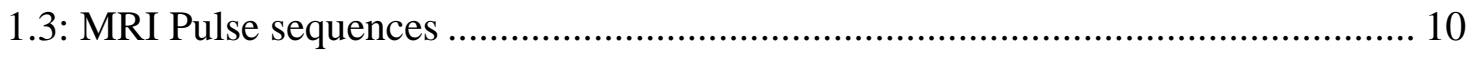

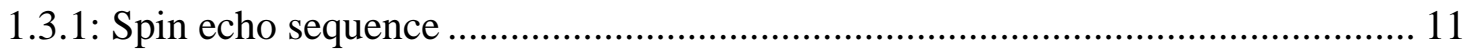

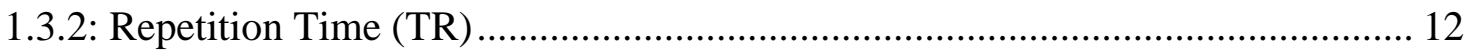

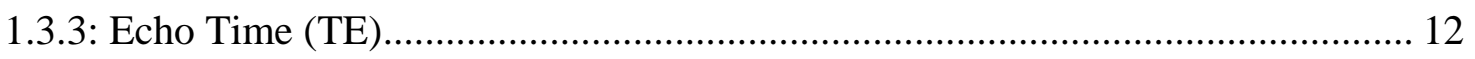

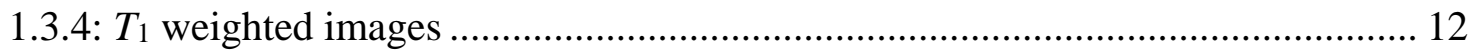

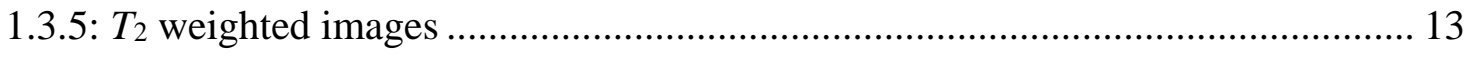

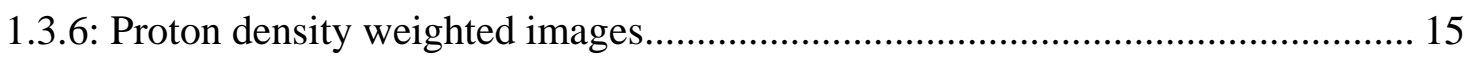

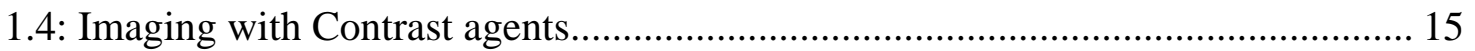

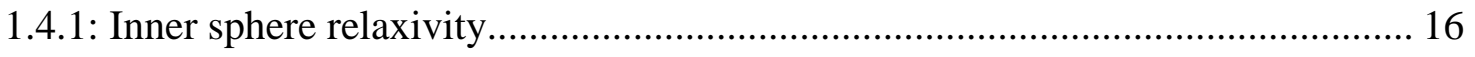

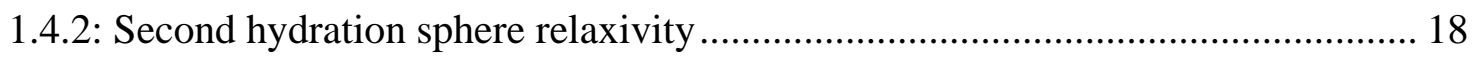

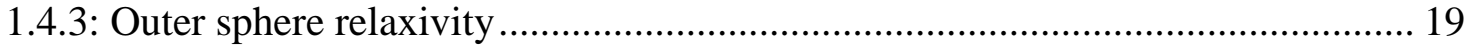

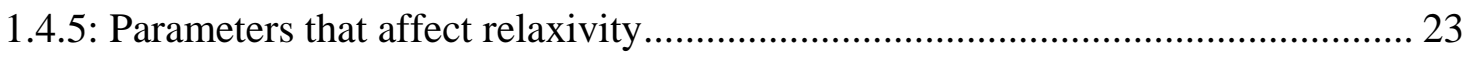

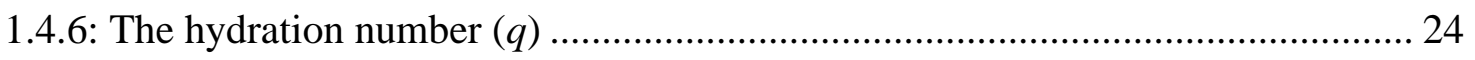




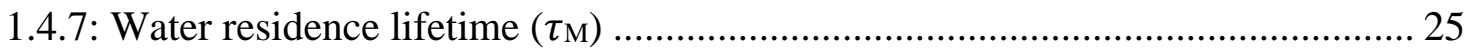

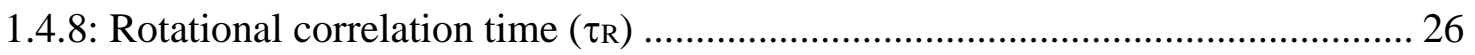

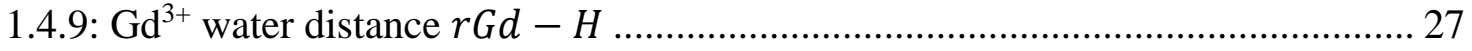

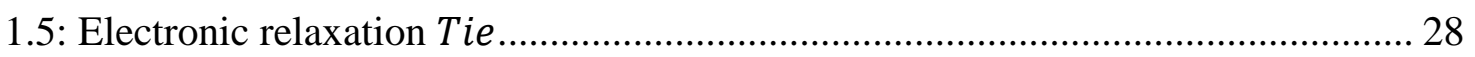

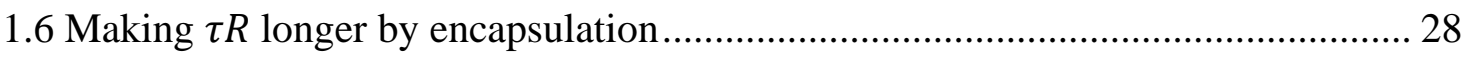

1.7: Chemical Exchange Saturation Transfer ........................................................ 29

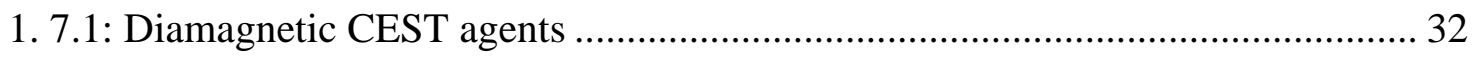

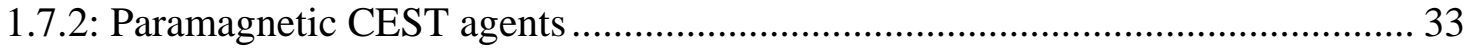

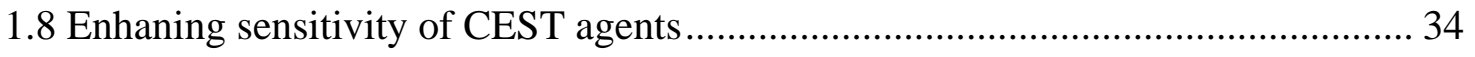

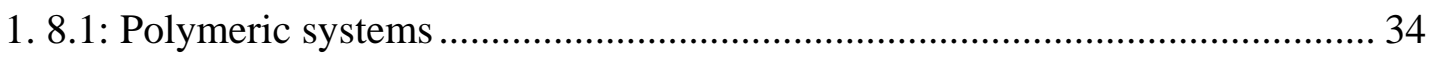

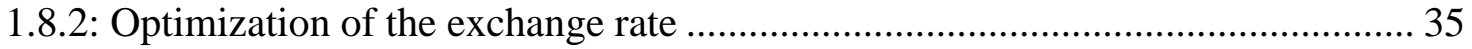

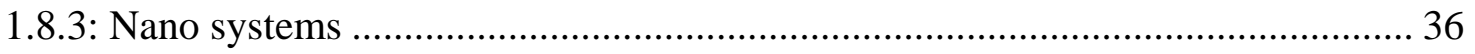

Chapter 2. Nanoassembled Capsules:A Versatile Platform for developing molecular

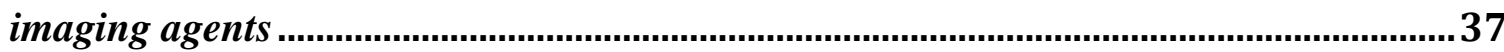

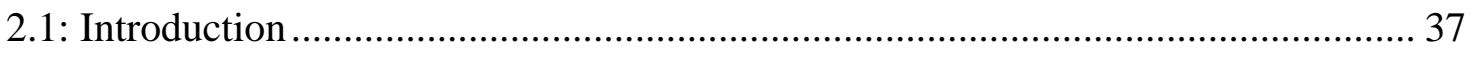

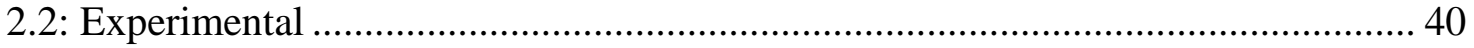

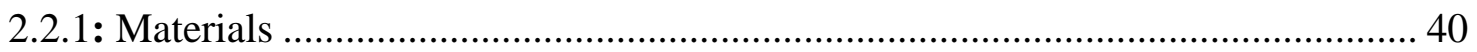

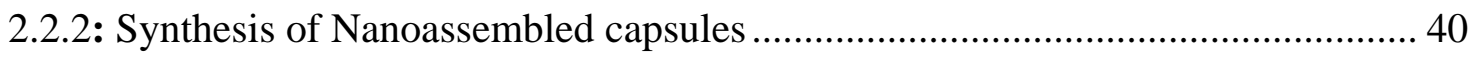

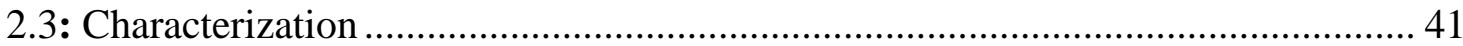

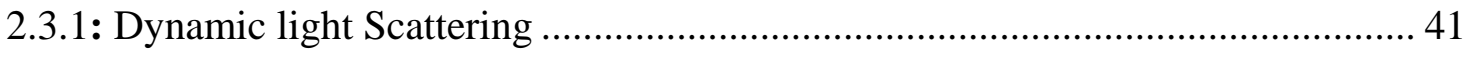

2.3.2: Scanning Electron Microscopy ............................................................... 41

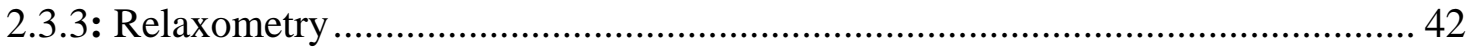

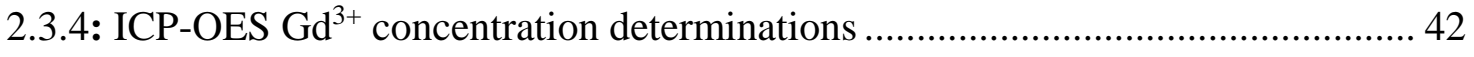

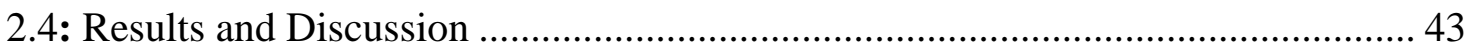

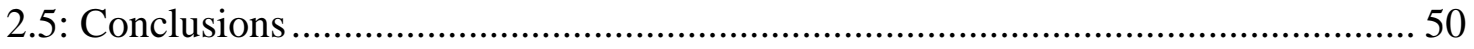

Chapter 3. An Alternative Coating Method for the High Payload Synthesis of Nano

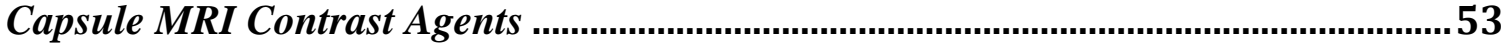

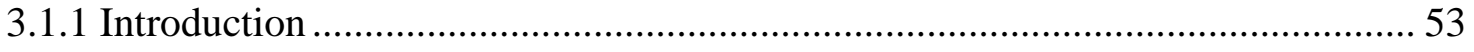

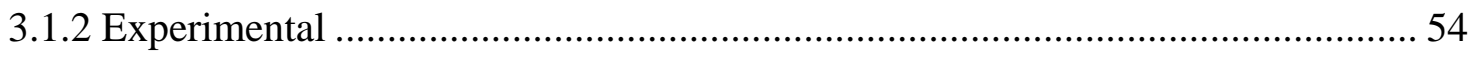

3.1.3 Synthesis of CACs with Simple Diamagnetic Anions ..................................... 55 


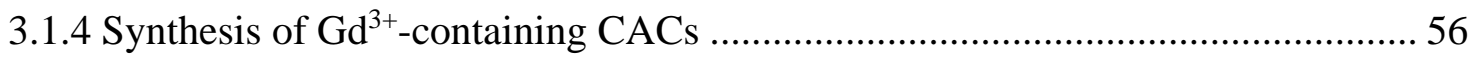

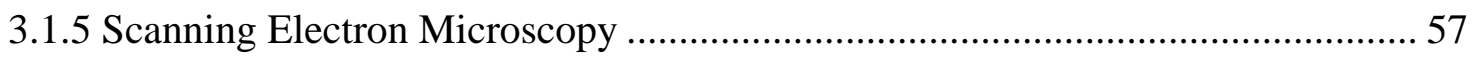

3.1.6 Transmission Electron Microscopy ………………........................................ 57

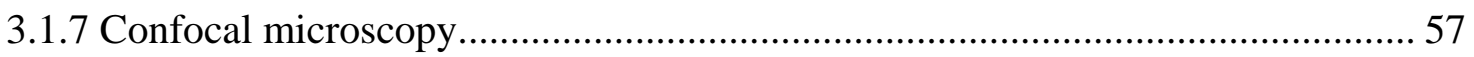

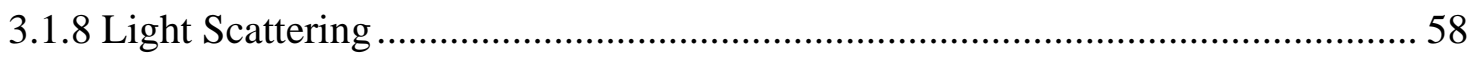

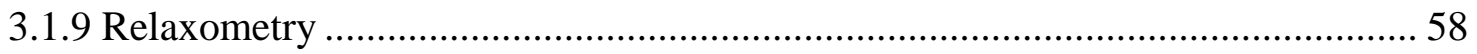

3.1.10 ICP-OES Gd ${ }^{3+}$ Concentration Determinations............................................... 58

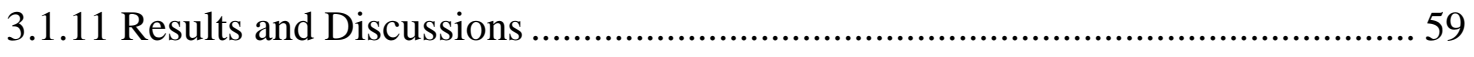

3.1.12: Probing the stability of cross linked assembled capsules (CACs) ..................... 63

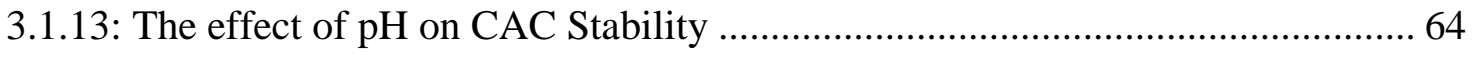

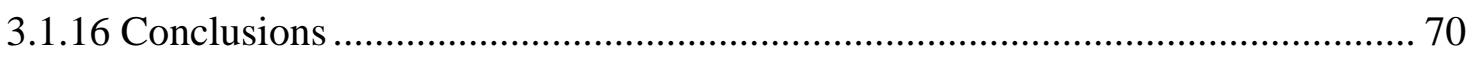

Chapter 4. Synthesis of a reverse NAC as a paraCEST agent ……............................72

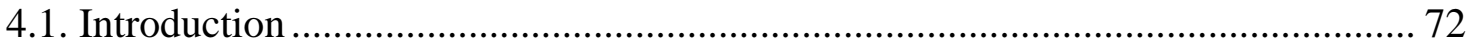

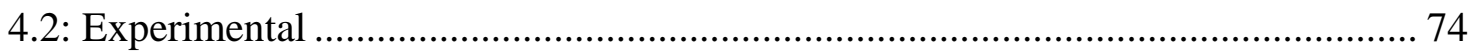

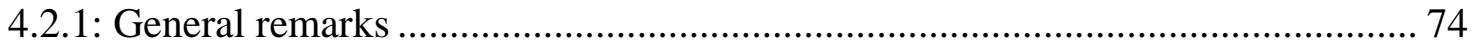

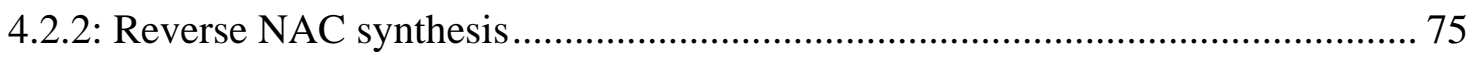

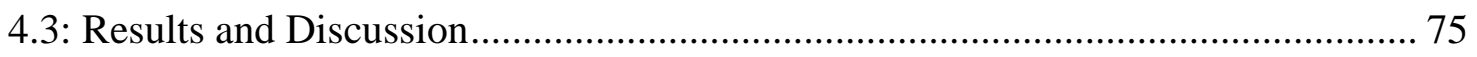

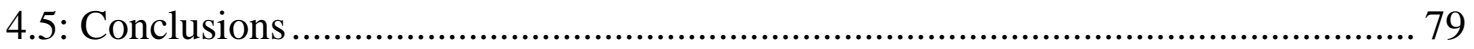

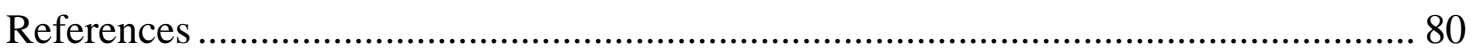


List of Tables

Table 1: Contrast in spin echo images is determined by the choice of TR and TE times 15

Table 2: Summary of the variations in size and relaxivity arising from changing the chelate charge and $R$ value during the preparation of NACs........................................ 48

Table 3: The average hydrodynamic diameter ( $\mu \mathrm{m})$ determined by dynamic light scattering of GdDOTP5- containing nano capsules (CACs and NACs) prepared with PAH in 3:2 MeCN/H2O as a function of the $R$ value used in their preparation. Data are presented with the $1^{\text {st }}$ population standard deviation ................................................... 63 
List of Figures

Figure 1.1: Illustration to represent spins in the absence (left) and presence (right) of a strong external magnetic field 5

Figure1.2: In the presence of static magnetic field, the spin magnetic moments and the resulting bulk magnetization precess at the Lamor frequency around the magnetic field 6 Figure1.3: Representation of the inversion recovery process. The magnetization vector MO in the equilibrium state. B) Inversion of Mo after $180^{\circ}$ pulse. C) Spins precessing in $x$ $y$ plane. D) Recovery of the magnetization factor along the z-axis. 7

Figure1.4: Principle of magnetic resonance imaging (MRI). A) Spins in the presence of external magnetic field. B) Spins precessing in $x$-y plane at the Larmor frequency upon applying a radiofrequency $C$ ) Spins decaying to their original z-axis. D) Spins back to their original z-axis.....

Figure1.5: Spin-spin relaxation describes the transverse component decay as a function of time and is characterized by $T_{2}$ 10

Figure1.6: Illustration of pulse sequence diagram for inversion recovery pulse sequence. A $180^{\circ}$ pulse sequence inversion is applied followed by a $90^{\circ}$ recovery pulse, as well as a $180^{\circ}$ repolarization pulse .......................................................................................... 11

Figure1.7: $T_{1}$ contrast difference between water and fat ............................................ 13

Figure1.8 : Axial brain MRI image of the same tissue showing how image weighting can be used to vary the contrast between grey matter, white matter and cerebrospinal fluid a) $T_{1}$ weighted $b$ ) proton density and $T_{2}$ weighted MRI images $^{26}$................................... 14

Figure1.9 :T2 contrast between fat and water ............................................................... 14

Figure1.10 : Schematic diagram showing the three hydration spheres, inner sphere water molecules are directly bound to the metal center; second sphere consists of water molecules coordinated to the ligand of the CA and the outer sphere water molecules.... 19 Figure1.11 : Contrast agents approved for clinical use, with common name and brand 22 Figure1.12 : CEST spectrum; A CEST effect (or CEST peak) can be observed at 10 ppm as highlighted with the arrow. The peak at 0 ppm is due to direct saturation of the bulk water protons by the RF saturation pulse. ${ }^{59}$

Figure 2.13: The effect of changing the $R$ value on the average hydrodynamic diameter of the NACs produced for GdDTPA ${ }^{2-}$ (purple), GdTTHA ${ }^{3-}$ (red) and GdDOTP ${ }^{5-}$ (green). Dashed lines are a guide to the eye only; error bars represent the $1^{\text {st }}$ standard deviation of the size distribution of the capsules. Sizes and their distributions are the average of 6 NAC preparations ${ }^{115}$

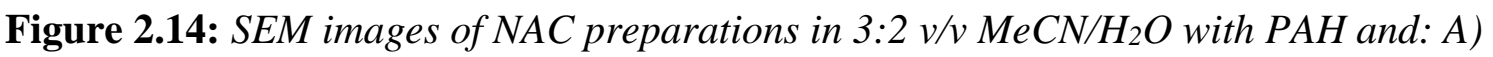
$\left.G_{D O O T A^{1-}} R=1.0 ; B\right) G d D T P A^{2-} R=0.75$; C) $\left.G^{2-} T T H A^{3-} R=0.75 ; D\right){G d D O T P^{5-}}^{-1}=$ 0.75 . 45 
Figure 2.15: The effect of changes in the overall $G d 3+$ chelate charge on the relaxivity of the NACs produced for different $R$ values....

Figure 2.16: Relaxivity values measured at $0.47 \mathrm{~T}$ at $25^{\circ} \mathrm{C}$ of encapsulated $\mathrm{Gd}$ chelates compared to the relaxivity values of the free chelates

Figure 3.17: Electron micrographs of different stages of CAC preparation. A) TEM image of a single pyrophosphate/PAH aggregate $(R=5)$ showing the loose morphology of the aggregate; B) TEM image of a pyrophosphate/PAH aggregate with a glutamate coating showing the tighter surface morphology of the coated aggregate; C) TEM image of a pyrophosphate/PAH CAC after treatment with EDC showing the crosslinked capsule shell; and SEM images of GdDOTP5-/PAH CACs after treatment with succinic acid and EDC D) $R=0.5$ and $E$ ) $R=1$. 60

Figure 3.18: A comparison of CACs (red) and NACs (green) prepared using PAH and GdDOTP5- average diameter. Each value is the mean of six nano capsule preparations

Figure 3.19: A comparison of CACs (red) and NACs (green) prepared using PAH and $\mathrm{GdDOTP}^{5-}$ contrasting the per $\mathrm{Gd}^{3+}$ longitudinal relaxivity $(20 \mathrm{MHz}$ and $298 \mathrm{~K})$. Each value is the mean of six nano-capsule preparations

Figure3.20: SEM images of CACs exposed to $\mathrm{pH}$ 3-11 from day 0 to day 28 for $R=0.3$

Figure 3.21: A comparison of CAC\% change in $G d 3+$ relaxivity and average size of capsules over 28 days at different $\mathrm{pH}$ values $\mathrm{pH} 3$ (red diamonds), $\mathrm{pH} 5$ (orange diamonds), pH 7 (green triangles), pH 9(blue circles), pH 11(purple circles) and salts $\mathrm{NaCl}$ (blue circles), $\mathrm{CaCl}_{2}$ (green squares) and $\mathrm{MgCl}_{2}$ (red squares .....

Figure 3.22: SEM images of CACs suspended in $5 \mathrm{mM} \mathrm{CaCl2,} \mathrm{MgCl} 2$ and $\mathrm{NaCl}$ for day 0 and day 28respectively.

Figure 3.23: Bright field optical field microscopy image of $R=0.5$ CACs prepared with $G_{D O T P}{ }^{-}$and treated with FITC after. 63xmagnification; excitation laser line $488 \mathrm{~nm}$; excitation wavelength $500 \mathrm{~nm}$; emission wavelength $555 \mathrm{~nm}$; scan format $512 \times 512$

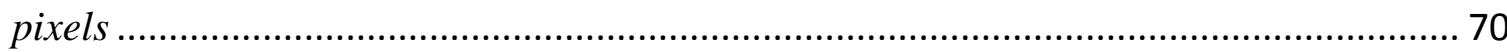

Figure 4.24: SEM images for reverse NACs for DyDOTAM/PAA for $R=0.5 \ldots \ldots \ldots \ldots \ldots . . .77$

Figure 4.25: CEST spectra of $40 \mathrm{mM}$ DyDOTAM chelate solution (red circles) and 40 mM DyDOTAM NACs (blue circles) 


\section{List of Schemes}

Scheme 1: Schematic presentation of the factors that affect relaxivity with one innersphere water molecule, $\square_{R}$ refers to the rotational correlation time of the molecule, $\square m$ mean water residence life time and $T_{1}, T_{2}$ relaxation times of the $G d^{3+}$ electron spin 23 Scheme 2:Schematic representation of spins aligned with and against the field (upper and lower energy levels, respectively) above simulated NMR spectra (below) for two chemically distinct pools of nuclei (left), two spins after a saturation pulse has been applied $^{66}$

Scheme 3: A Schematic representation of the preparation of nano assembled capsules which are $\mathrm{SiO}_{2}-\mathrm{NP}$-coated

Scheme 4: The structures of the chelates investigated in this work ${ }^{16}$ GdDOTA $^{1-}$, GdDTPA ${ }^{2-}$, GdTTHA $^{3-}$, and GdDOTP ${ }^{5-}$

Scheme 5: Schematic of synthesis of cross linked assembled capsules versus that of NACs

Scheme 6: The structure of the chelate used in the synthesis of the reverse NACs 
List of Equations

Equation 1: Boltzmann distribution .................................................................. 4

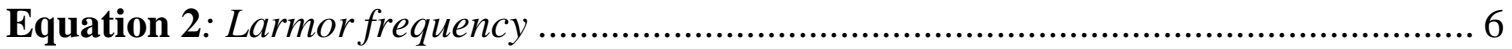

Equation 3: Contribution of hydration spheres to overall observed relaxivity ............... 16

Equation 4: Inner hydration sphere relaxivity .................................................... 16

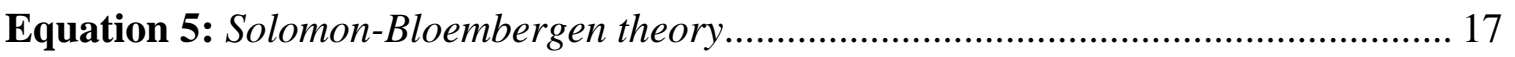

Equation 6: Solomon-Bloembergen-Morgan equations ........................................... 17

Equation 7: Outer sphere relaxivity Solomon-Bloembergen equations ........................ 20

Equation 8: Einstein model for diffusion for rigid spheres ....................................... 20

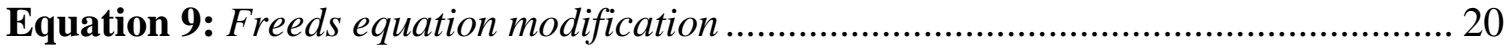

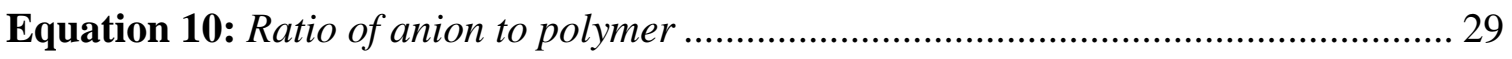

Equation 11: Exchange rate for CEST exchange rates .............................................. 30 


\section{Chapter 1: Introduction}

1.1 A look back at where we have been and where we are

Medical imaging has revolutionized medicine, since the discovery of X-rays in 1895 by Roentgen $^{1}$. Various imaging modalities have been developed which include computerized tomography (CT), optical imaging; ultra sound and nuclear imaging (such as PET and SPECT) are some of the well-known rapidly growing and frequently used medical diagnostic tools. ${ }^{2}$ X-ray imaging utilizes high frequency characteristics of electro-magnetic rays which when passed through human body they are absorbed in different amounts depending on the density of the tissue. Even though the X-rays are quick and non-invasive the ionizing radiation has several side effects ${ }^{3}$, high dose of Xrays can damage DNA in cells and increases the risk of getting cancer by about $0.08 \%$. In the mid-1970s CT came into use; it is an X-ray based technique developed by Godfrey Hounsfield. It is a combination of a computer and X-ray technologies, used to provide cross sectional images of the body. It was Lazaro Spallanzani who started the study related to ultra sound in 1794 and Karl Dussik in 1942 introduced ultrasound into the field of diagnostics. It is a medical imaging technique that uses high frequency sound waves and their echoes. It has an advantage of low cost $t^{4}$ and is noninvasive also it does not require ionizing radiation ${ }^{5-7}$, versatile ${ }^{8}$ and portable ${ }^{9-13}$. Ultra sound is mainly used as part of routine checkup in case of pregnancy to check fetus in womb. In the 1950s nuclear imaging techniques entered the field of diagnostic imaging test. Nuclear imaging methods employ radioactive tracers which ultimately cause the emission of gamma rays. Nuclear imaging is a combined study of physics, chemistry, mathematics, computer 
science and medicine. Nuclear imaging commonly used in detecting various infections and injuries ${ }^{4}$. It is considered a non-invasive technique ${ }^{5}$. Positron Emission Tomography (PET) and Single Photon Emission Computed Tomography (SPECT) are types of nuclear imaging. In PET the tracer emits a positron when it decays, when the positron collides with an electron they mutually annihilate and two gamma rays are simultaneously emitted. $^{14,} 15$ In SPECT radioactive tracer itself emits a gamma ray. In both cases detection of the gamma rays allows an image to be built up. ${ }^{16,17}$ The major disadvantage of nuclear medicine is the use of radioactive tracers and cost.

In the 1970s Magnetic Resonance Imaging also evolved. At first resistive magnets with weak magnetic fields were used, producing images with low spatial resolution. Despite this it still had a better soft tissue discrimination than $\mathrm{CT}$ allowing earlier diagnosis ${ }^{18}$. In the late 1980s the super conducting magnets became popular 1.5 Tesla. MRI has the added advantage over X-Ray based $\mathrm{CT}$ for not requiring ionizing radiation and MRI has a high spatial resolution compared to the other techniques ${ }^{15},{ }^{19}$, it can be used to evaluate heart, kidneys, liver, blood vessels ${ }^{20}$ and other body tissues ${ }^{17}$. All the diagnostic techniques have now grown from a state of infancy to a high level of maturity. ${ }^{21}$

\subsubsection{A history of MRI}

Since William Roentgen first discovered the X-ray phenomenon in $1895^{22}$ medical imaging techniques have become irreplaceable tools in the diagnosis and treatment supervision. Before the development of the imaging modalities the ability to see inside the human body historically was to have surgery. One key advance in the development 
of modern imaging techniques came in 1822 when the French mathematician and physicist Joseph Fourier proposed that a function could be represented by a series of sinusoids. The resulting mathematical transformation, later named Fourier transform, forms the basis of Magnetic Resonance Imaging. A further concrete step towards MRI was the Austrian physicist Wolfgancy Pauli's prediction of the existence of nuclear spin, in 1924. The concept of nuclear spin is central to the development of nuclear magnetic resonance methods. At Columbia University a group led by Isidor Isaac Rabi showed that an oscillating magnetic field could be used to induce transitions between nuclear spin states of lithium and chlorine atoms in a molecular beam eventually leading to the award of a Nobel Prize in physics in 1944. The extension of Rabi's 1938 observation of NMR in a beam of independent molecules to solid and liquid samples was successfully independently and simultaneously in 1948 Felix Bloch ${ }^{23}$ and Edward Purcell observed that signal is emitted from a sample in a magnetic field when it is irradiated with an electromagnetic energy of a particular frequency. They shared the Nobel Prize in physics in 1952 for this discovery of condensed phase magnetic resonance. Although Rabi's work was crucial as the conceptual and technical leap achieved by Bloch and Purcell really set the stage for the development of modern NMR and MRI. Independently Henry C Torrey and Erwin L Hahn contributed a final critical component of the modern NMR, they demonstrated the feasibility of pulsed NMR, an idea originally suggested by Bloch and observed free Larmor precession. Independently Paul Lauterbur, Raymond Damadian and Peter Mansfield introduced methods to allow images of the human body to be acquired, including slice selection and fast acquisition. Research in MRI was largely 
restricted to academic laboratories during the 1970s. William Edelstein a post-doctoral fellow in Mallard's group implemented Fourier imaging and obtained the first clinically useful image of a human subject. All demonstrated the imaging potential of NMR and MRI was finally born. In 2003 Lauterbur and Mansfield were awarded Nobel Prize in medicine for their development of magnetic resonance imaging. As a non-invasive imaging tool Magnetic Resonance Imaging (MRI) is less harmful than other imaging modalities that use ionizing radiation or radioactive tracers, such as X-Rays and PET.

\subsection{Basic principle of Magnetic Resonance}

Magnetic resonance imaging is one of the most widely used techniques in diagnosis, clinical medicine and biomedical research. The basis of MRI is nuclear magnetic resonance. ${ }^{1} \mathrm{H}$ is the most favorable nucleus for MRI because of its abundance in biology (water), and its high gyromagnetic ratio. When a human body is placed in a large magnetic field the magnetic moments of the proton nuclear spins tend to align slightly either with or against the magnetic field (Figure 1.1). There is a slight predominance of nuclei in the lower energy state (slightly more aligned closely with the magnetic field), than there are in the high energy state (slightly more aligned against the field). This produces a small net magnetic moment, the magnitude of which can be described by the Boltzmann equation (Eqn 1), which can be probed with radiofrequency radiation.

$$
\frac{N_{-1 / 2}}{N_{+1 / 2}}=e^{-\Delta E / k T}
$$

\section{Equation 1: Boltzmann distribution}


Where $\Delta \mathrm{E}$ is the energy difference between the two levels and $\mathrm{k}$ is the Boltzmann constant and $\mathrm{T}$ is the temperature in Kelvin.
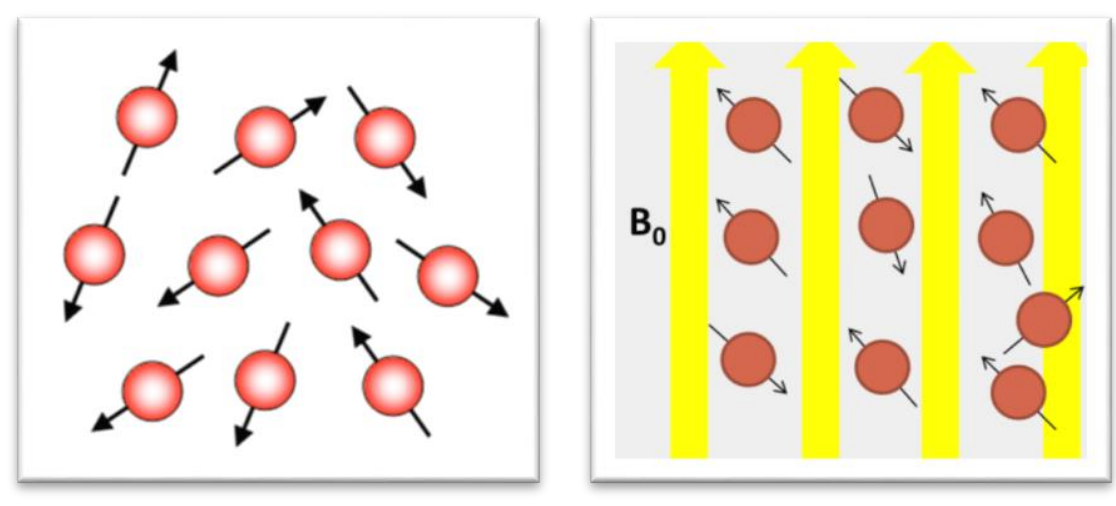

Figure 1.1: Illustration to represent spins in the absence (left) and presence (right) of a strong external magnetic field

The magnetic dipole moment of a nucleus is directly proportional to the spin angular momentum, nuclear spin angular momentum is a fundamental property that makes a nucleus a continuously rotating positive charge, and as a moving charge it has an associated magnetic field and magnetic dipole moment and the magnetic dipole moment is directly proportional to the spin angular momentum. It is the gyromagnetic ratio that relates the nuclear rotating frequency to the strength of the magnetic field. For hydrogen the gyromagnetic ratio is $42.6 \mathrm{MHz} / \mathrm{T}$. Nuclei of hydrogen contain a single proton giving them a net nuclear spin of $1 / 2$. Its high gyromagnetic ratio and its high biological and isotopic abundance make the proton ideal for MRI. In the absence of a strong external magnetic field individual nuclear magnetic moments are randomly distributed and precess incoherently. However, in a strong external magnetic field a radio frequency pulse can be used to apply torque to the net magnetic moment causing coherent precession. The frequency at which the spins precess is called the Larmor frequency $(\omega)$ 
and is given by the product of the gyromagnetic ratio $(\gamma)$ and the strength of the applied magnetic field $(B)$ (Eqn 2). Gyrometric ratio relates nuclear rotating frequency to the strength of the magnetic field and is expressed in terms of $\mathrm{MHz}$ per tesla in MRI

$$
\omega=\gamma B
$$

\section{Equation 2: Larmor frequency}

Even though individual spins obey the laws of quantum mechanics, the behavior of a group of spins experiencing the same magnetic field strength is best described using classical mechanics. Therefore the vector sum of the magnetization vectors from all spin packets is represented by a net magnetization $\mathrm{M}_{0}$.

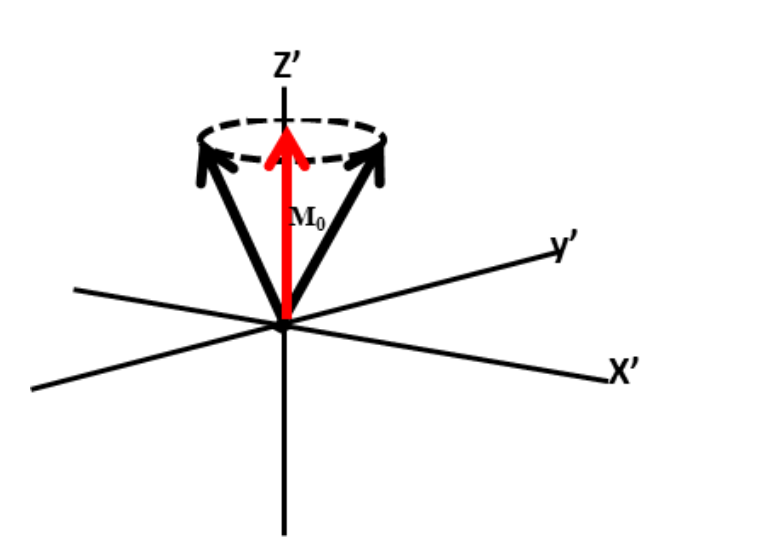

Figure1.2: In the presence of static magnetic field, the spin magnetic moments and the resulting bulk magnetization precess at the Lamor frequency around the magnetic field At equilibrium the net magnetization vector aligns with the main magnetic field along the Z-axis. On applying a radio frequency pulse $\mathrm{B}_{1}$, at or near the Larmor frequency of the protons. The magnetization vector is "tipped" such that component lies in the transverse (x-y) plane. Precession of the net magnetization vector around the z-axis at the Larmor frequency induces a current in the receiver coil, which after amplification and processing 
afford the NMR signal. This process is independent of the return to equilibrium also known as relaxation.

\subsection{1: Inversion recovery pulse sequence}

Inversion recovery pulse sequence is used to measure $T_{1}$. It begins with a $180^{\circ}$ inverting pulse which flips the initial longitudinal magnetization $\left(\mathrm{M}_{0}\right)$ to point antiparallel the main magnetic field $\mathrm{B}_{0}$. After switching the $180^{\circ}$ radio frequency off, the net magnetization begins to recover to its equilibrium position. At a time interval ( $\mathrm{t}$ ) after the $180^{\circ}$ pulse a $90^{\circ}$ interrogation pulse is acquired, this rotates the longitudinal magnetization into the $\mathrm{x}-\mathrm{y}$ plane. This allows the extent of recovery of the longitudinal relaxation to be read out; repeating this exercise while varying the value of the time interval $t$ allows a recovery curve to be generated from which the longitudinal relaxation time constant, $T_{1}$, can be determined..$^{24}$

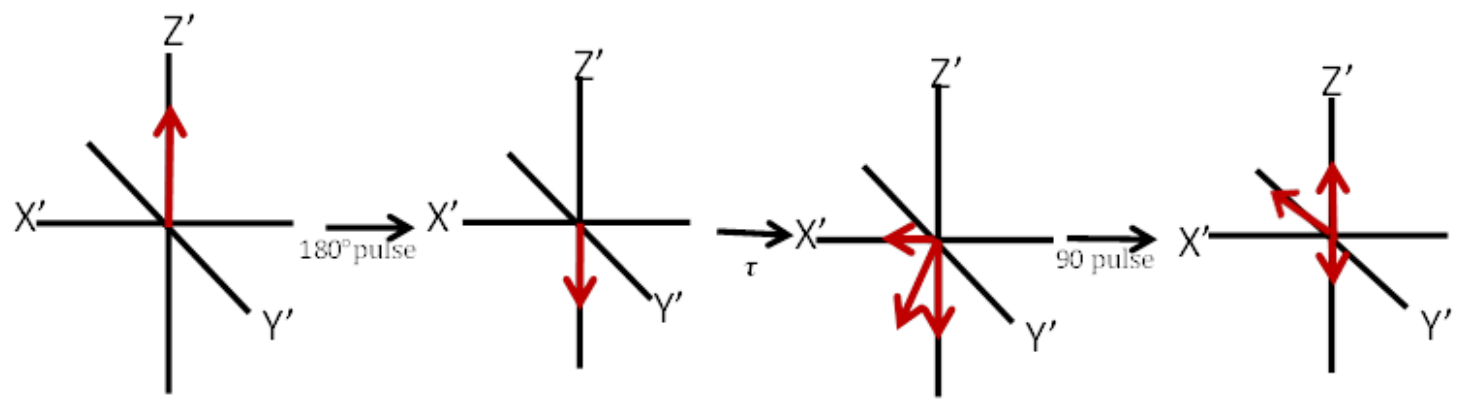

Figure1.3: Representation of the inversion recovery process. The magnetization vector $M 0$ in the equilibrium state. B) Inversion of Mo after $180^{\circ}$ pulse. C) Spins precessing in $x$ $y$ plane. D) Recovery of the magnetization factor along the z-axis

\subsubsection{Relaxation}

After the radiofrequency pulse is turned off the excited nuclear spins begin to return to their original stable equilibrium state. This process is facilitated by modulating local 
magnetic fields. The net magnetization vector $\mathrm{M}_{0}$ gradually returns to lie along the $\mathrm{z}$-axis (longitudinal relaxation). Concurrently there is dephasing (loss of coherence) of the net magnetization vector (transverse relaxation). These two processes are two types of relaxation in NMR sometimes called spin-lattice (longitudinal relaxation) and spin-spin (transverse relaxation).

\subsection{3: Spin-Lattice relaxation, $T_{1}$}

Spin lattice relaxation, also called longitudinal relaxation is the recovery of net magnetization towards z-axis and involves an exchange of energy between spin systems and surrounding or lattice.

Rotational and vibrational motion modulates local magnetic fields in the lattice and some components of this magnetic field have a frequency equal to the Lamor frequency and can therefore facilitate nuclear relaxation. Relaxation arises from this dipole-dipole interactions. Energy rate loss indicates how closely spins are coupled to the lattice. Hydrogen in the form of water is a small molecule so it moves more rapidly, while large molecules like fat molecules move much more slowly. $T_{l}$ relaxation time shows the relationship between the frequency of these molecules and the Lamor frequency. If these frequencies are the same, $T_{1}$ recovery of $\mathrm{M}_{\mathrm{o}}$ is rapid and if they are different the $T_{1}$ recovery is slow as a result different tissues exhibit different $T_{l}$ recovery curves. Spinlattice relaxation is described by an exponential function characterized by a time constant $T_{1}$, the period for the longitudinal magnetization to recover $63 \%$ of its equillibrium value. 


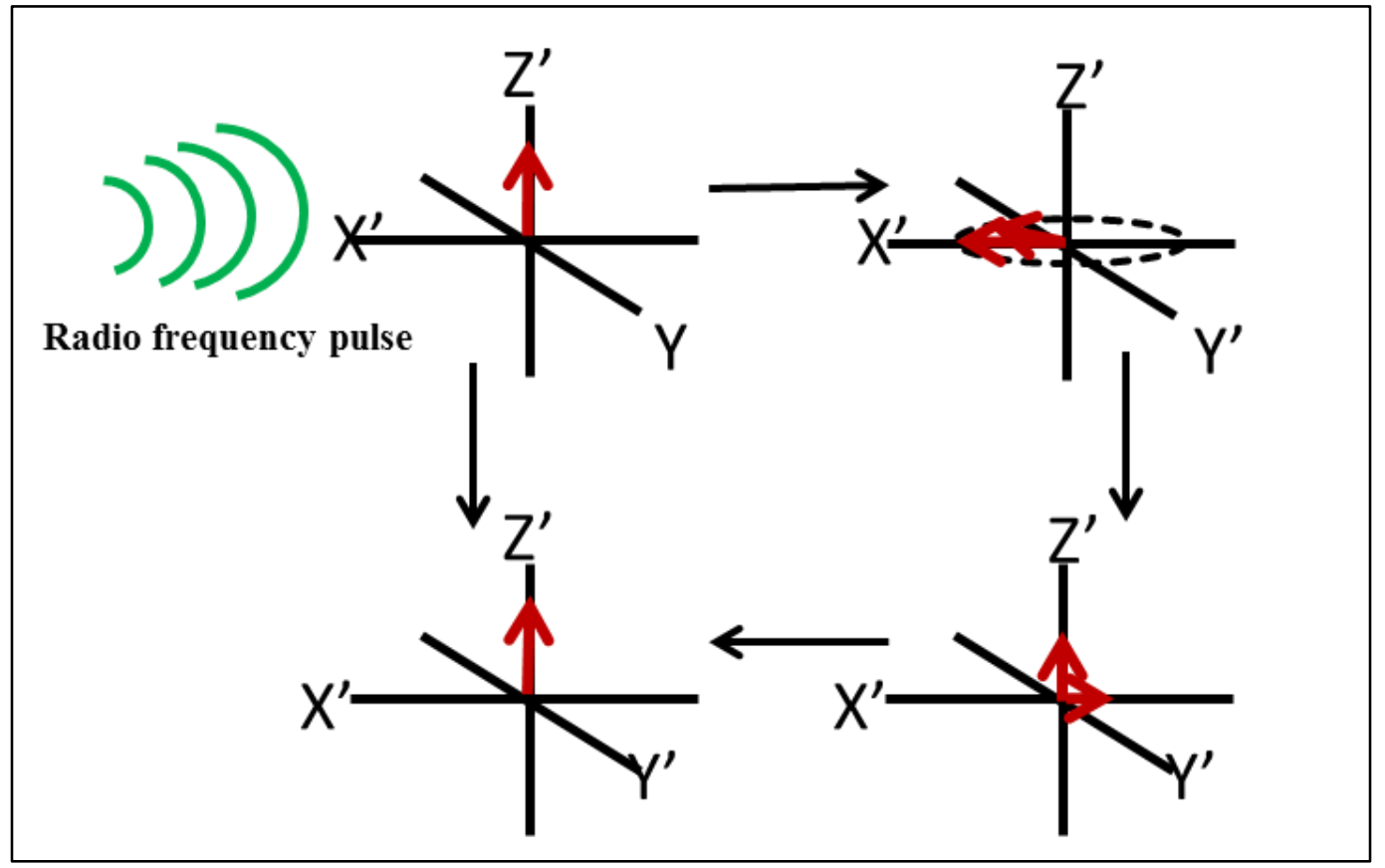

Figure1.4: Principle of magnetic resonance imaging (MRI). A) Spins in the presence of external magnetic field. B) Spins precessing in $x$-y plane at the Larmor frequency upon applying a radiofrequency C) Spins decaying to their original z-axis. D) Spins back to their original z-axis

\subsection{4: Spin- Spin relaxation, $T_{2}$}

Transverse relaxation is also called spin-spin relaxation. After a $90^{\circ}$ pulse is applied the net magnetization vector is in the transverse plane. When the radio frequency pulse is switched off each individual spins that makes up the net magnetization vector lie in transverse plane but they do not all experience exactly the same local magnetic field. This means that they will precess at slightly different rates and the population of spins begins to dephase or lose phase coherence. This loss of coherence means that the magnitude of the net magnetization vector decreases leading to a loss of signal. This process is also dependent on the rate of molecular motion and so its rate also varies between tissues. Larger macromolecules like in fat experience more static internal 
magnetic fields and consequently more rapid transverse relaxation. Spin-spin relaxation is described by an exponential decay function characterized by the time constant $T_{2}$, as shown in Figure 1.5.

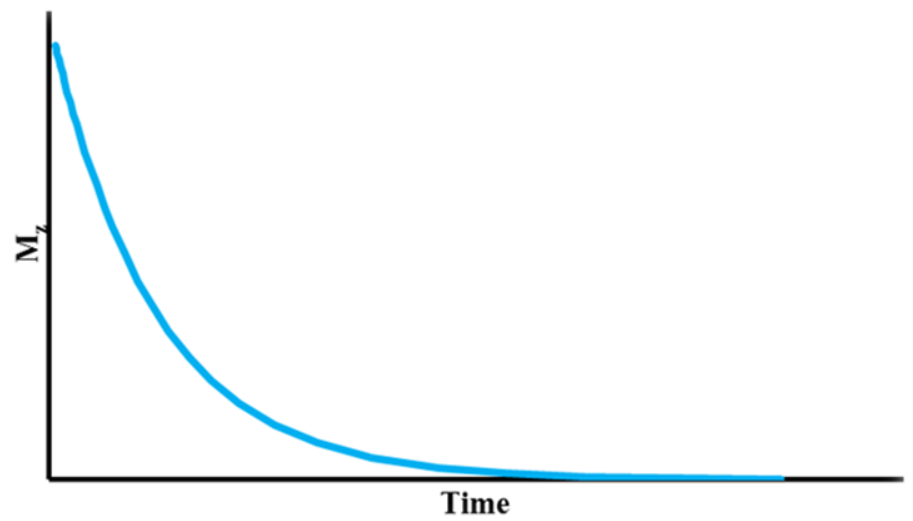

Figure1.5: Spin-spin relaxation describes the transverse component decay as a function of time and is characterized by $T_{2}$

\subsection{5: Relaxivity}

The efficacy of a contrast agent on relaxation is often expressed in terms of relaxivity either $r_{1}$ or $r_{2}$ to describe the effect on longitudinal and transverse relaxation, respectively. Relaxivity is defined as the relaxation rate constant (either $1 / T_{1}$ or $1 / T_{2}$ ) per millimolar concentration of the contrast agent.

\section{3: MRI Pulse sequences}

An MRI pulse sequence is a set of RF pulses applied to a sample to produce a specific form of MRI signal. It is programmed with a set of changing magnetic gradients and each sequence has a set of parameters which are described in an MRI protocol. 
Repetition time (TR) and Echo time (TE) are parameters that are varied to highlight or suppress different tissues, reduce imaging times and reduce or avoid artifacts. There are two major sequences in MRI: the spin echo and the gradient echo.

\subsection{1: Spin echo sequence}

In a spin echo sequence, the spins are excited by a $90^{\circ}$ pulse. After a short time a $180^{\circ}$ radio frequency pulse is applied which reverses the phase of precession causing the dephasing magnetization vector to rephrase. This creates an echo which occurs at the echo time $\mathrm{TE}\left(\mathrm{TE}=2 \times\right.$ the time between the $90^{\circ}$ and $180^{\circ}$ pulses). This helps to reduce the effects of magnetic field inhomogeneities. The magnitude of the echo depends on value of $\mathrm{T}_{2}{ }^{*}$ which reflects magnetic field homogeneity. Gradient echo pulse sequences differ from the spin echo in that they can use field gradients, rather than a radiofrequency pulse, to generate the echo.

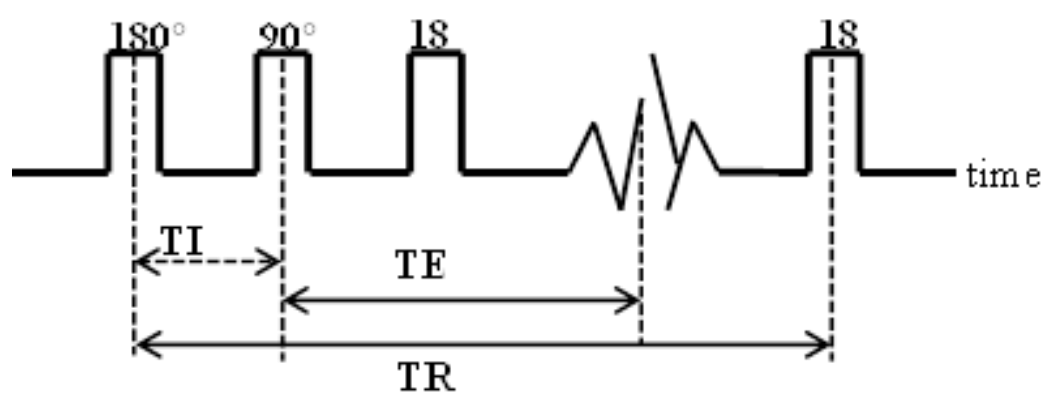

Figure1.6: Illustration of pulse sequence diagram for inversion recovery pulse sequence. A $180^{\circ}$ pulse sequence inversion is applied followed by a $90^{\circ}$ recovery pulse, as well as a $180^{\circ}$ repolarization pulse 


\subsection{2: Repetition Time (TR)}

The Repetition Time, TR, determines how much longitudinal magnetization recovers between each radio frequency pulse. It is the time from the application of an excitation pulse to the application of the next pulse. If the repetition time is shorter than the time it takes to fully recover longitudinal magnetization returns to its equilibrium position then the signal will be attenuated. The extent of attenuation will depend upon the $T_{1}$ relaxation time constant.

\subsection{3: Echo Time (TE)}

The Echo time is time between the application of radio frequency excitation pulse and the peak of the echo signal induced by the $180^{\circ}$ pulse (Figure 1.6). An underlying limitation of MRI is that measurement of signal is not possible immediately after the application of the excitation pulse therefore the signal must be preserved by a refocusing (echo) pulse. The magnitude of the echo signal is dependent on the echo time. The longer the echo time the more magnetization is lost unrecoverable to dephasing and the lower the signal.

Varying the repetition and echo times are the primary means by which image contrast is manipulated. In spin echo sequences there are three main parameters that affect the weighting of an image: $T_{1}, T_{2}$ and proton density.

\subsection{4: $T_{1}$ weighted images}

Image contrast can be adjusted by varying the repetition time and echo time values. The selection of a short TR will prevent complete recovery of magnetization in tissues with shorter $T_{1}$ relaxation time constants. A short $\mathrm{TE}$ ensures that the maximum 
magnetization is retained in the transverse plane and the effect of $T_{2}$ is minimized. This is known as $T_{1}$-weighting - it results in image contrast that is more dependent on differences in $T_{1}$ than differences in $T_{2}$. The effect of $T_{1}$ weighting on an MRI of the brain can be seen in Figure1.8. it can be seen for example that fat has a shorter $T_{1}$ (because it has a relatively slow tumbling rate which is similar to that of the Larmor frequency) and thus appears with stronger signal intensity while water, which has a relatively longer $T_{1}$ (due to relatively high tumbling rate which does not match the Larmor frequency), appears with lower signal intensity on $T_{1}$ weighted MR images.

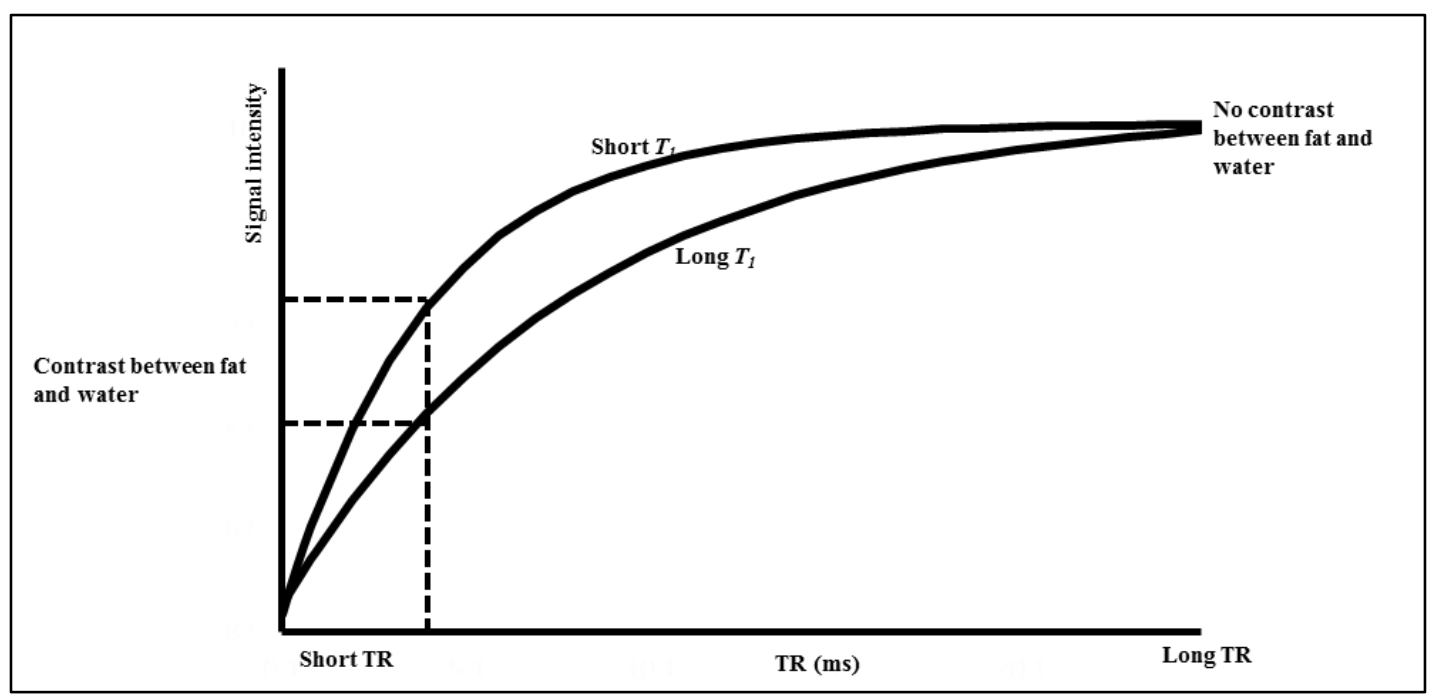

Figure1.7:T $T_{1}$ contrast difference between water and fat

\subsection{5: $T_{2}$ weighted images}

If TR is made long then the effect of $T_{1}$ on image contrast is minimized. The image can be made to reflect differences in $T_{2}$ between tissues if a longer echo time is selected. Longer $T_{2}$ values mean that more coherent magnetization remains in the transverse plane when the echo pulse is applied and this leads to a stronger signal. A shorter $T_{2}$ value (e.g. 
$10-20 \mathrm{~ms})^{25}$ causes more loss of coherence and therefore a weaker echo signal. Images acquired with long TR and TE values reflect differences in tissue $T_{2}$ and are therefore known as $T_{2}$-weighted images. When selecting a long repetition time and a short echo time, the dependence of image contrast on $T_{1}$ and $T_{2}$ becomes insubstantial and contrast is instead determined by proton density of the tissues.

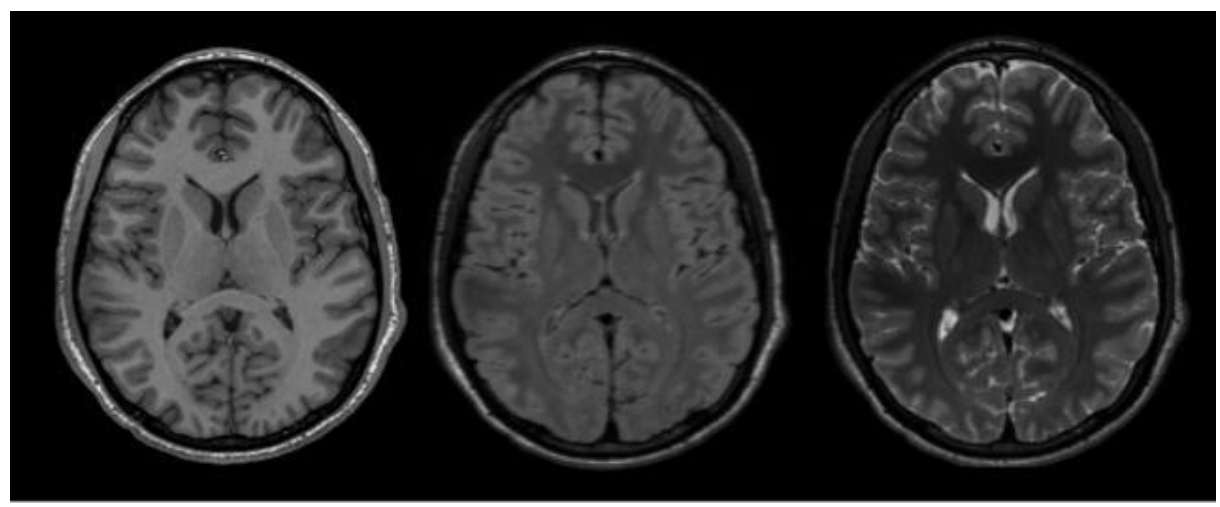

Figure1.8 : Axial brain MRI image of the same tissue showing how image weighting can be used to vary the contrast between grey matter, white matter and cerebrospinal fluid a) $T_{1}$ weighted $b$ ) proton density and $T_{2}$ weighted MRI images ${ }^{26}$

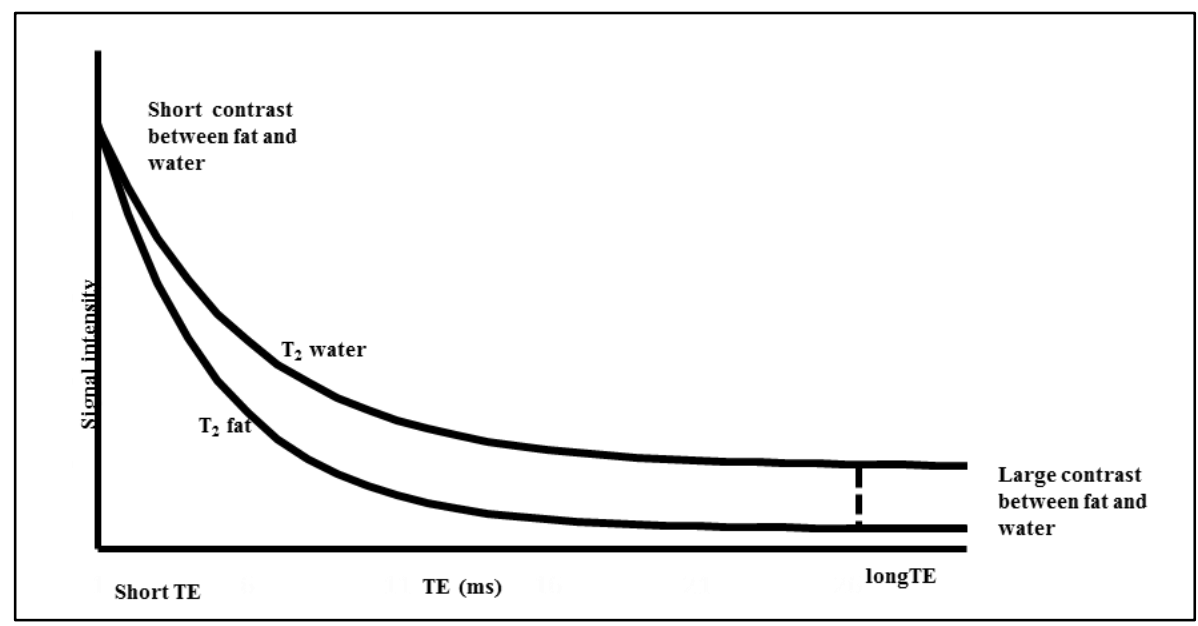

Figure1.9: $T_{2}$ contrast between fat and water 
1.3.6: Proton density weighted images

Besides the differences in relaxation times in different tissues, differences in the proton density per unit volume can also contribute to contrast in MR images. Proton density images are collected such that the effects of relaxation are minimized: i.e. TE $\ll T_{2}$ and TR $\gg T_{1}$. Under these circumstances contrast will therefore mainly reflect proton density. Tissues with a high proton concentration will appear bright like brain tissues whilst tissues with low hydrogen content will appear dark e.g. cortical bone.

Table 1: Contrast in spin echo images is determined by the choice of TR and TE times

\begin{tabular}{ccc}
\hline Spin Echo imaging & Short $\boldsymbol{T}_{\mathbf{2}}$ & Long $\boldsymbol{T}_{\mathbf{2}}$ \\
\hline Short TR & $T_{1}$ weighted & - \\
Long TR & Proton density & $T_{2}$ weighted \\
\hline
\end{tabular}

\section{4: Imaging with Contrast agents}

Sometimes the inherent contrast in MRI is insufficient for the proper characterization of the anatomy or pathology of interest, even after applying appropriate pulse sequences. In these cases, a contrast agent can help amplify the differences between healthy and pathological regions. The major reason of using contrast agents is to increase the relaxation rate constant of water protons in the tissue to which it is distributed. This is achieved by use of a paramagnetic element. Bloch and co-workers reported use of ferric nitrate to enhance the relaxation rate constants of water protons and 30 years later Lauterbur and co-workers used $\mathrm{Mn}^{2+}$ salts to differentiate water phantoms based on their relaxation rate constants. The fluctuating magnetic field around the paramagnetic center of the contrast agent administered due to unpaired electrons is responsible for increasing 
the relaxation rate constant of nearby water protons. The relaxivity of a contrast agent is a measure of its ability to increase the relaxation rate constant of water. The relaxivity of a contrast agent is described by a model that considers three contributions: the inner coordination sphere (which is defined by the exchange of water directly coordinated to the metal center); the outer hydration contribution (consisting of water molecules that are not directly coordinated to the metal center but are diffusing close to the metal ion); and the second hydration sphere (defined by exchange of protons that are weakly associated with the chelate but not bound to the metal ion). ${ }^{27}$ The overall relaxivity is made up from contributions from each of these solvation spheres according to Eqn 3.

$$
r_{1}^{o b s}=r_{1}^{I S}+r_{1}^{S S}+r_{1}^{O S}
$$

\section{Equation 3: Contribution of hydration spheres to overall observed relaxivity}

\subsection{1: Inner sphere relaxivity}

The relaxation arising from exchange of inner sphere water molecules can be described by the Solomon- Bloembergen-Morgan (Eqns 4, 5, and 6). When one or more water molecules are coordinated to a paramagnetic metal ion the largest contribution to the overall relaxivity comes from these molecules exchanging with the bulk water according to Eqn 4. Currently, all clinically available contrast agents are gadolinium based chelated with organic chelators polyaminocarboxylates with one exchanging water molecule.

$$
r_{1 p}^{I S}=\frac{[C] q}{55.6}\left(\frac{1}{T_{1 M}+\tau_{M}}\right)
$$

Equation 4: Inner hydration sphere relaxivity 
$q$ is the number of water molecules which are coordinated to the central metal ion (hydration number), $C$ is the molar $\mathrm{Gd}^{3+}$ ion concentration, $T_{I M}$ is the relaxation time constant of the bound water protons and $\tau_{M}$ is the mean residence time of the protons bound to the water molecules in the inner coordination sphere. The value $\mathrm{T}_{1 \mathrm{M}}$, can be obtained by using Solomon-Bloembergen-Morgan (SBM) theory (Eqns 5 and 6$)^{27}$.

$$
\frac{1}{T_{1 M}}=\frac{2}{15} \frac{\gamma_{H}^{2} g^{2} S(S+1) \beta^{2}}{r_{G d H}{ }^{6}}\left[\frac{3 \tau_{c 1}}{1+\omega_{H}^{2} \tau_{c 1}^{2}}+\frac{7 \tau_{c 2}}{1+\omega_{S}^{2} \tau_{C 2}^{2}}\right]
$$

Equation 5: Solomon-Bloembergen theory

$$
\frac{1}{\tau_{C i}}=\frac{1}{\tau_{R}}+\frac{1}{\tau_{M}}+\frac{1}{T_{1 e}} \quad i=1,2
$$

Equation 6: Solomon-Bloembergen-Morgan equations 
$S$ is the electron spin quantum number, $\gamma$ is the proton nuclear magnetogyric ratio, where $\omega_{\mathrm{H}}$ and $\omega_{\mathrm{s}}$ are the proton and electron larmor frequency respectively, $\beta$ and $\mathrm{g}$ are the electronic factor and Bohr magneton, respectively. $r_{G d H}$ is the distance between the metal ion and the protons of the coordinated water molecules, $\tau_{c i}(i=1,2)$ are the correlation times of the modulation of dipolar electron-proton coupling, the correlation time $\tau_{c i}$ of paramagnetic metal complex reflects weighted contributions from, $\tau_{\mathrm{R}}$, the rotational correlation time of the $\mathrm{Gd}^{3+}$ chelate, $T_{\mathrm{ie}}$ the electronic relaxation time of the $\mathrm{Gd}^{3+}$ ion and $\tau_{M}$ the mean residence life time.

\subsection{2: Second hydration sphere relaxivity}

Water molecules not directly coordinated to the metal centre may also contribute to a relaxation enhancement from the contrast agent. The second hydration sphere consists of water molecules that are weakly asscoiated with the contrast agent. For example hydrogen bonding interactions with the ligand tend to increase the residence time close to the paramagnetic ion. SBM theory also describes the contributiom of second sphere relaxivity and the same parameters govern second sphere relaxivity as inner sphere relaxivity. However, unlike the inner sphere water, the number of the second sphere water molecules be quite large without decreasing the densicitity of the chelating ligand, reducing the chelate's thermodynamic stability. The life time of hydrogen bonded water molecules in the second sphere can be very short, groups that can increase this life time will promote second sphere relaxivity. 


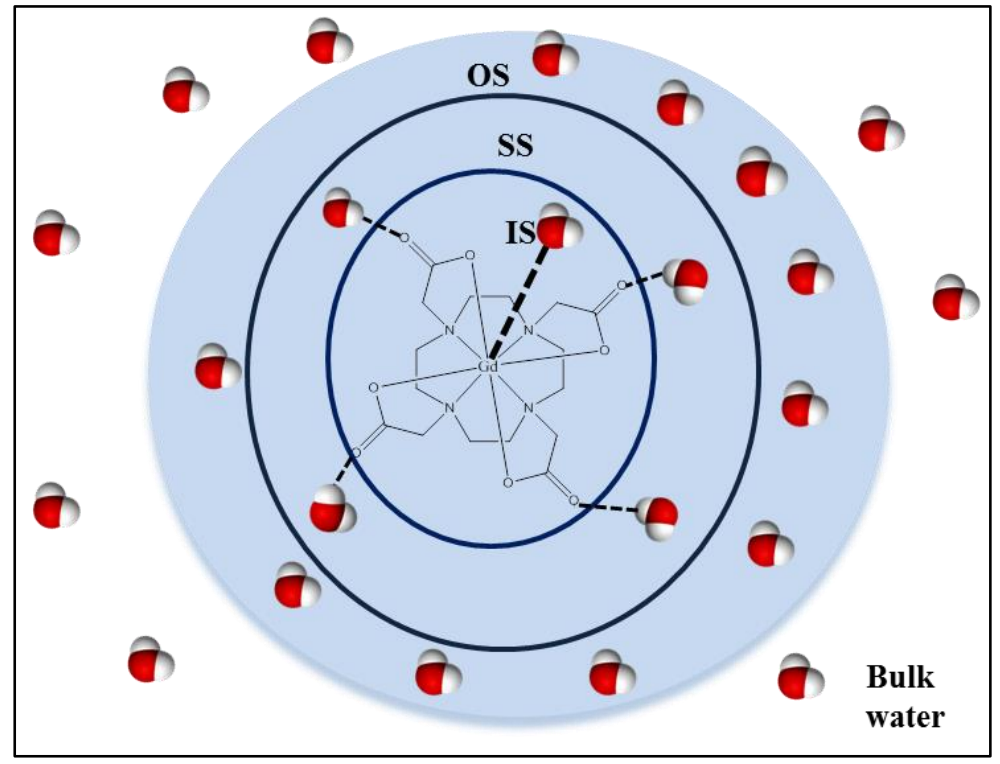

Figure1.10 : Schematic diagram showing the three hydration spheres, inner sphere water molecules are directly bound to the metal center; second sphere consists of water molecules coordinated to the ligand of the CA and the outer sphere water molecules

\subsection{3: Outer sphere relaxivity}

The contribution of the outer sphere has been shown to be approximately $1.8 \mathrm{mM}^{-1} \mathrm{~s}^{-1}(20$ $\mathrm{MHz}, 25^{\circ} \mathrm{C}$ ) of total relaxivity ${ }^{5}$. The main determinants of outer sphere relaxivity are the relative diffusion coefficient of the dissolved substance and solution $(D)$, the distance of closest approach $(a)$ and the electronic relaxation rate constants of the paramagnetic ion. The mechanism of outer sphere relaxivity is essentially the same those of the second and inner spheres: modulation of dipole-dipole interactions between the electronic spin and the proton spin, however in this case the distance are greater and timing is governed by the rate of diffusion of the water molecules in close proximity to the paramagnetic centre. The diffusion of the paramagnetic chelate in solution is usually described by the Freed equations. ${ }^{28,29}$ The general form of theory of outersphere relaxivity has similarities with the SBM equations as shown in Eqn 7. 


$$
\frac{1}{T_{1(o s)}}=\frac{C_{\pi} N_{s} \gamma_{H}^{2} \gamma_{S}^{2} \hbar^{2} S(S+1)}{d^{3} \tau_{D}}
$$

Equation 7: Outer sphere relaxivity Solomon-Bloembergen equations

Here C is a numerical constant, $N_{S}$ is the number of metal ions per cubic centimeter, $₫$ is the relative translational diffusion time, and $\gamma_{H}$ and $\gamma_{S}$ are diffusion constants of water proton and the metal complex, respectively. Furthermore, if molecular motion, described by the Stokes-Einstein model, is considered then diffusion can be approximated as the diffusion of rigid spheres in a medium of viscosity $(\eta)$ (Eqn 8).

$$
\mathrm{D}=\frac{k T}{6 \pi a \eta}
$$

\section{Equation 8: Einstein model for diffusion for rigid spheres}

For small paramagnetic metal chelates the Freeds equation can be modified as in Eqn 9 .

$$
R^{o s}=\quad c^{o s}\left(\frac{1}{a D}\right)\left[7 \mathrm{~J}\left(\omega_{s}\right)+3 \mathrm{~J}\left(\omega_{H}\right)\right]
$$

\section{Equation 9: Freeds equation modification}

For $\mathrm{q}=1$ systems like GdDOTA ${ }^{1-}$ and GdDTPA ${ }^{2-}$ the overall contribution from the outer hydration sphere is $40-50 \%{ }^{30}$. Contribution from outer sphere hydration is more pronounced in chelates like GdTETA ${ }^{1-}$ and $\operatorname{GdTTHA}^{3-}(q=0$ chelates), which have no inner sphere contribution and relatively small second sphere contributions. In the case of a $q=0$ chelate such as GdDOTP ${ }^{5-}$ the outer sphere contibution is close to half of the 
overall relaxivity, with the remainder arising from a large second sphere contribution facilitated by the phosphonates groups coordinated to the paramagnetic centre.

\subsection{4: Contrast agents}

Contrast agents are not directly detected but their effect on the surrounding water molecules, in the form of increases to the rate of relaxation that are detected. Contrast agents change the intensity of the water proton signal in appropriately weighted images. $\mathrm{Gd}^{3+}$ is the most frequently used paramagnetic ion due to its particularly favorable characteristics; the high spin state (seven unpaired electrons) of the $3+$ oxidation state and the long electronic relaxation time constants, which are much longer (approximately $10^{-9}$ s) than for the other lanthanides such as $\mathrm{Dy}^{3+}, \mathrm{Ho}^{3+}$ and $\mathrm{Eu}^{3+} .{ }^{31}$ Unfortunately, free $\mathrm{Gd}^{3+}$ ion is toxic in vivo. The use of $\mathrm{Gd}^{3+}$ in chelate becomes form is therefore mandatory in biomedical applications to reduce toxicity and increase solubility of free lanthanide aqua ions at physiological $\mathrm{pH}$. The toxicity of $\mathrm{Gd}^{3+}$ arises due to the comparable ionic radii of $\mathrm{Gd}^{3+}$ to $\mathrm{Ca}^{2+}$ which causes the lanthanide to interfere with the $\mathrm{Ca}^{2+}$ signaling channels. ${ }^{32}$ Chelation reduces relaxivity since it decreases the number of coordination sites available for water proton exchange, from 8-9 sites for aquated $\mathrm{Gd}^{3+}$ ions compared to 1-2 sites for $\mathrm{Gd}^{3+}$ chelates. Clinically available contrast agents (Figure 1.11) tend to have comparitively low relaxivity, approximately $4 \mathrm{mM}^{-1} \mathrm{~s}^{-1}\left(20 \mathrm{MHz}, 25^{\circ} \mathrm{C}\right)$. This is much lower than the theoretically predicted maximum, ${ }^{33}$ assuming that all the parameters that affect relaxivity are optimized. The $\mathrm{Gd}^{3+}$ chelates used in clinical MRI are derived from linear and macrocyclic polyaminocarboxylates such as DTPA(diethylenetriaminepentaacetic acid), DOTA (1,4,7,10-tetraazacyclododecane- 
1,4,7,10-tetra acetate). ${ }^{34}$ These chelates have extremely high thermodynamic stability constants $\log K_{G d L}=23.6$ and 25.3 for GdDTPA $^{2-}$ and GdDOTA $^{1-}$, respectively, in addition to retaining one vacant coordination site for an inner sphere water molecule.

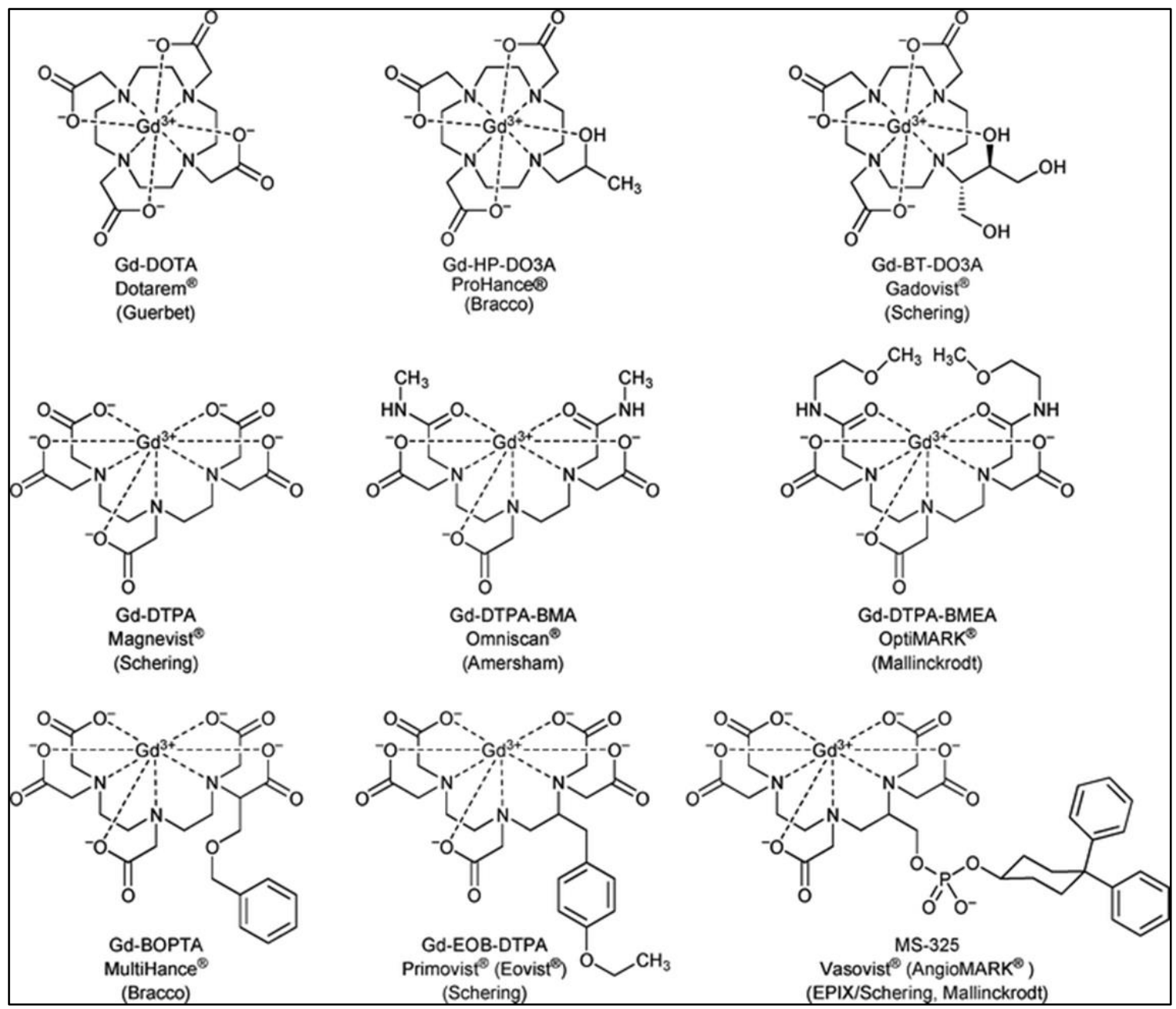

Figure1.11 : Contrast agents approved for clinical use, with common name and brand They have extremely high $\mathrm{Gd}^{3+}$ thermodynamic formation constants $\log K_{G d L}=23.6$ and 25.3 respectively and a coordination site for an inner sphere water molecule. 
1.4.5: Parameters that affect relaxivity

The theory of $T_{1}$ contrast agents clearly shows that numerous parameters affect relaxivity as shown in scheme 1 . The hydration number $(q)$, the rotational correlation time $\left(\tau_{\mathrm{R}}\right)$ and the water residence life time $\left(\tau_{\mathrm{M}}\right)$ can all be adjusted by altering the chemical environment around the paramagnetic ion. Altering these parameters can have a significant effect on the overal relaxivity value of the chelates.

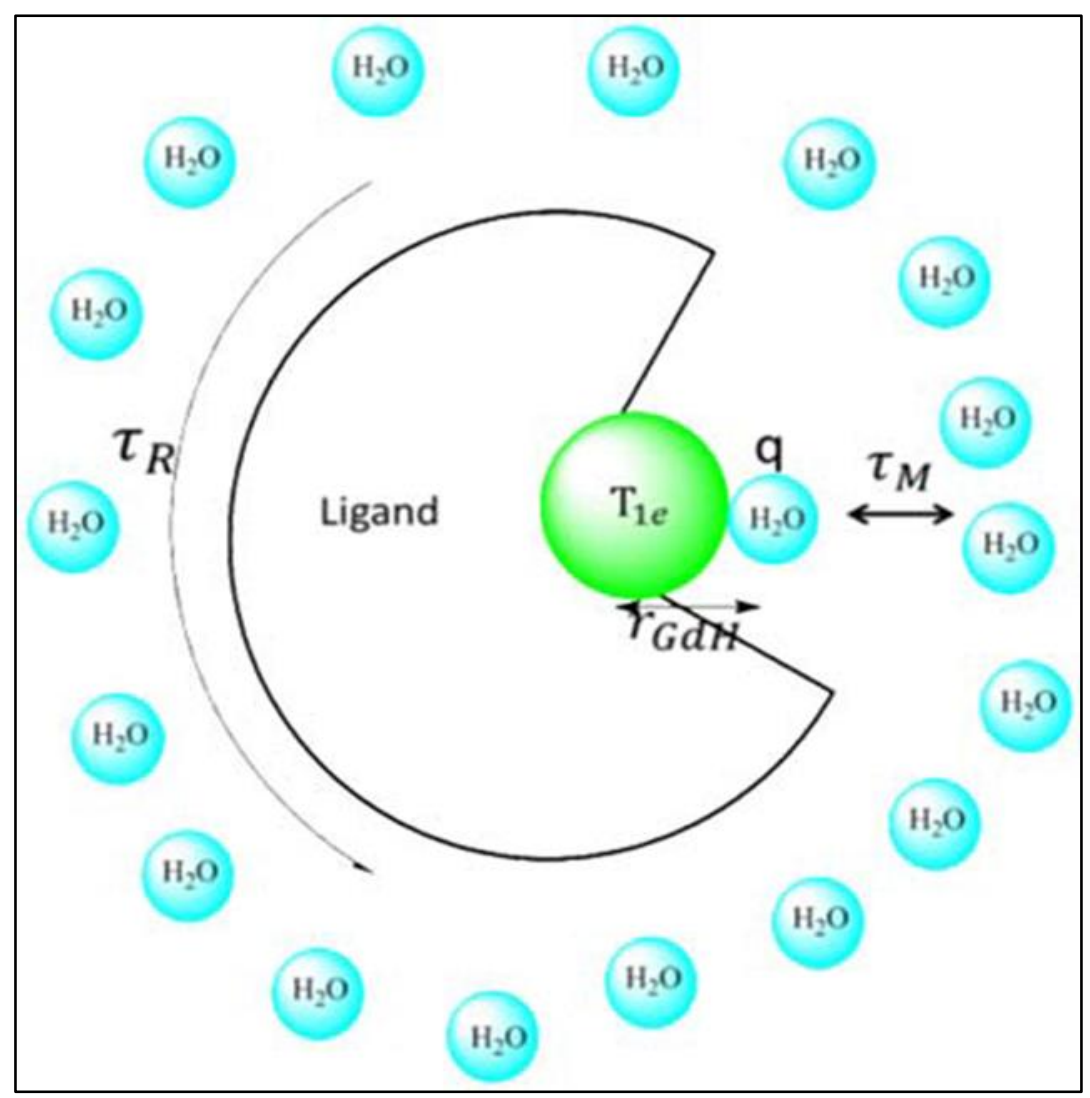

Scheme 1: Schematic presentation of the factors that affect relaxivity with one innersphere water molecule, $\tau_{R}$ refers to the rotational correlation time of the molecule, $\tau m$ mean water residence life time and $T_{1}, T_{2 e}$ relaxation times of the $G d^{3+}$ electron spin 


\subsection{6: The hydration number $(q)$}

The hydration number $(q)$ is the number of water molecules that can directly coordinate to the paramagnetic metal ion. The inner sphere relaxation increases linearly with an increasing $q$. Current clinical contrast agents utilize polyamino-carboxylate chelating ligands that bind $\mathrm{Gd}^{3+}$ with a coordination number of eight, leaving one available coordination site for the binding of water. The reason for this is the necessity to balance both stability and relaxivity while designing contrast agents. Although there are new chelates being tested which have more than one water molecule coordinated to the metal centre. ${ }^{35}$ There is always an underlying concern over the use of contrast media with hydration numbers greater than 1 . Surprisingly, despite possessing no inner sphere water, chelates like GdDOTP ${ }^{5-}$ have higher relaxivity than $q=1$ chelates like GdDOTA'. The phosphonates groups on GdDOTP ${ }^{5-}$ can interact with an estimated 6 water molecules in the second sphere and keep them bound for longer ( $50 \mathrm{ps})$ than second sphere waters in carboxylate chelates. For these reasons the contribution of outer sphere hydration more than compensates for the absence of an inner hydration sphere. ${ }^{36}$

Efforts have been undertaken to increase the number of water molecules directly coordinated to the metal centre. GdDOTA has been modified to increase $q=1$ to $q=2$ by removal of an acetate group creating a seven coordinate complex GdDO3A. The relaxivity of GdDO3A is $\mathrm{r}_{1}=4.8 \mathrm{mM}^{-1} \mathrm{~s}^{-1}\left(20 \mathrm{MHz}, 40^{\circ} \mathrm{C}\right)$, under the same conditions GdDOTA $^{1-}$ has a relaxivity of $3.5 \mathrm{mM}^{-1} \mathrm{~s}^{-1} \cdot{ }^{37}$ The overall difference of relaxivity is attributed to an increase in hydration number. However, this increase in relaxivity comes at the cost of a deacrease in the thermodynamic stability constant by three orders of 
magnitude. ${ }^{38,39}$ Ken Raymond and co-workers have devoloped recently a ligand with a hydration number of two and rather than traditional poly(amino-carboxylate) complexes these ligands are comprised of all oxygen donors and are based on hydroxy pyridinone (HOPO) ligands. Gd-TREN-1-Me-3-2-HOPO is the first such ligand that provides two positions for water coordination without compromising thermodynamic stability ${ }^{40}$.

\subsection{7: Water residence lifetime $\left(\tau_{\mathrm{M}}\right)$}

Water proton exchange is a key factor in governing relaxivity because the relaxation effect is transferred to the bulk water protons through water exchange between the paramagnetic center and the surrounding bulk water. However, relaxivity can be limited due to water exchange that is either too fast or too slow. Fast water exchange results in a very small water residence life time $\tau_{M}$ of the water molecule coordinated to the paramagnetic center which is usually not long enough to allow water to be properly relaxed. Water exchange that is too slow will also result in poor relaxivity due to poor transmission of the relaxation effect from the paramagnetic center to the bulk water. An optimal value of $\tau_{M}$ is dependent on magnetic field strength and $\tau_{R}$ of the complex.

In lanthanide chelates, water can be in exchange occur through an associative or dissociative mechanism. In $\mathrm{Gd}^{3+}$ aqua ion is an eight/nine coordinate system which exchanges through an associative pathway. However, in nine-coordinate mono-hydrated exchange can occur only through a dissociative mechanism since there is no availlable site for a second water to bind. Complex charge, solvent accessibility, steric effects around the water binding site of chelates are all key factors that govern $\tau_{\mathrm{M}}$. Negatively charged $\mathrm{Gd}^{3+}$ complexes of DOTA or DTPA-like ligands demonstrate shorter residence 
life time than neutral or positively charged chelates. $\tau_{\mathrm{M}}$ should be short, in the range of $10-30 \mathrm{~ns}$, for macromolecular contrast agents at $60 \mathrm{MHz}$ or $1.5 \mathrm{~T}$. For higher fields a $\tau_{M}$ in the range of 3-10 ns is predicted to be optimal ${ }^{41}$. The polyaminocarboxylate chelates used as clinical contrast agents exchange more slowly than this. Merbach and coworkers increased steric crowding of mono aqua GdDTPA ${ }^{2-}$ and GdDOTA $^{1-}$ chelates by substituting one or two of the ethylene bridges with propylene bridges ${ }^{42}$. Despite having a small effect on the stability of the chelate this induced steric effect on the $\mathrm{Gd}^{3+}$ center facilitates the leaving of the bound water, resulting in an increase in water exchange rate from about 30-50 fold for GdDOTA ${ }^{1-}$ analogues and $40-50$ fold for GdDTPA analogues. Square antiprismatic (SAP) or twisted square antiprismatic (TSAP) isomers observed in Ln (DOTA) complexes and their derivatives have different values. Reports display 10-100 times faster water exchange for TSAP isomer when compared to SAP. $^{43-46}$

\subsection{8: Rotational correlation time $\left(\tau_{\mathrm{R}}\right)$}

Molecular tumbling usually relates to molecular size. The relaxvity of a $\mathrm{Gd}^{3+}$ chelate increases with increasing $\tau_{R}$. The minimum value of $\tau_{R}$ that will afford an increase in relaxivity depends on the magnetic field and occurs most efficiently between the Lamour frequencies of $10-70 \mathrm{MHz}$ and when $\tau_{\mathrm{M}}$ is between $10-50 \mathrm{~ns}$. A number of strategies have been used to reduce the tumbling rates of contrast agents by covalent or noncovalent attachment to macromolecules. ${ }^{47}$ dendrimers, liposomes ${ }^{48,} 49$ carbohydrates, micelles $^{50}$ and solid nanoparticles. ${ }^{51,52,}$ Self-assembled $\mathrm{Fe}\left[\mathrm{Gd}_{2} \text { bpy(DTTA) } 2\left(\mathrm{H}_{2} \mathrm{O}\right)_{4}\right]^{53}$ agents displayed four times higher in vivo relaxivity than the commercially available 
GdDOTA $^{1-}$. When the clinical agent MS-325 binds to human serum albumin (HSA) using a hydrophobic diphenylcyclohexyl moiety relaxivity increases 8 fold at $20 \mathrm{MHz}$ and $37^{\circ} \mathrm{C} \cdot{ }^{40,54}$ But these approaches have an added advantage of allowing the attachment of multiple contrast agents to to be attached to each carrier, further increasing the relaxivity both by slow tumbling of the $\mathrm{Gd}^{3+}$ chelate and an increase $\mathrm{Gd}^{3+}$ payload. The binding scheme should be chosen carefully in order to attain the desired $\tau_{R}$ value without affecting the other more favorable properties of the $\mathrm{Gd}^{3+}$ ion complexes, such as the water exchange rate $\tau_{M}$ Recently, the model free Lipari- Szabo approach has proved useful in describing the rotational motion of macromolecule. Two kinds of motions are assumed to affect relaxation: a rapid, local motion and a slower global motion. Even though it is a crucial parameter, there is no direct method to measure $\tau_{\mathrm{R}}$. It can be obtained by fitting the data from ${ }^{1} \mathrm{H}$ Nuclear Magnetic Relaxation Dispersion (NMRD) profile.

\subsection{9: $\mathrm{Gd}^{3+}$ water distance $r_{G d-H}$}

$T_{1 \mathrm{M}}$ is proportional to $1 / \mathrm{r}^{6}$, as shown in Eqn 3 above, therefore varying the $\mathrm{Gd}^{3+}$-water distance could lead to drastic enhancement of relaxivity. Decreasing the distance by about $0.2 \AA$ would increase relaxivity by $50 \%$. Gd-H distances have been determined by X-ray studies or calculated indirectly by relaxivity studies and they are generally in the range of $2.5 \AA$ - $-3.3 \AA$. The $\mathrm{Gd}-\mathrm{H}$ distance for GdDOTA ${ }^{1-}$ and GdDTPA ${ }^{2-}$ derivatives has been determined as $3.1 \AA ̊ \pm 0.1 \AA ̊$ by ENDOR spectroscopy. ${ }^{55},{ }^{56}$ 


\section{5: Electronic relaxation $T_{i e}$}

Electronic relaxation for $\mathrm{Gd}^{3+}$ chelates is related to the zero field splitting of the electronic spin states and its dynamic distortion. ${ }^{57}$ In a paramagnetic ion unpaired electrons under going relaxation generate a flactuating magnetic field that causes nuclear relaxation. ${ }^{58}$ This defines how efficiently the proton will be relaxed. For $\mathrm{Gd}^{3+}$ electronic relaxation depends on the magnetic field applied and its importance decreases as the magnetic field increases. $\mathrm{Gd}^{3+}$ has a symmetrical electronic configuration and this gives rise to relatively slow electronic relaxation but for elements like $\mathrm{Dy}^{3+}$ (even though it has a large magnetic moment) it is a poor relaxation agent since the electronic relaxation is fast.

\subsection{Making $\tau_{R}$ longer by encapsulation}

Nano assembled capsules (NACs) are structures in which polyanions and cationic polymers are assembled into a stable closed structure encapsulated by a nano-particle shell. It has been shown that $\operatorname{GdDOTP}^{5-}$ can be employed as the polyanion in this system and be trapped inside the capsule. ${ }^{59}$ This approach is attractive because formation of aggregates occurs at very mild $\mathrm{pH}$ values and at room temperature; the nanocapsules self assembly technique is extremely facile and quickly produces the nanoassembled capsules; the technique is able to effectively slow tumbling of the $\mathrm{Gd}^{3+}$ chelate and affords high per- $\mathrm{Gd}^{3+}$ relaxivity. ${ }^{60}$ NACs have been synthesized using different cationic polymers such as polyallylamine hydrochloride (PAH), poly-L-lysine (PLL) ${ }^{61}$ and polyethylenimine $(\mathrm{PEI})^{62}$ and using multivalent anions such as phosphate citrate ${ }^{63}$, EDTA $^{64}$, indocyanine green ${ }^{65}$ and the chelate ${ }^{62}$ e.g. GdDOTP ${ }^{5-}{ }^{62}$ Encapsulation within 
NACs affords larger gains in relaxivity when compared to the clinically available chelates, the method comes with attractive features like the capability to modify the surface by attaching targeting molecules on the surface of the nanoassembled capsules. In addition it allows for the use of higher payloads of the chelate while at the same time reducing molecular tumbling of the small chelates thereby increasing the relaxivity values attained. The per $\mathrm{Gd}^{3+}$-relaxivity was found to be dependent upon the the size of the NAC, smaller NACs afforded higher relaxivity. The size of the NAC can easily be manipulated by changing the charge ratio $R$, which is defined as the ratio of the total negative charge of the anion to the total positive charge of the polymer as shown below in Eqn $10 .{ }^{62}$

$$
\boldsymbol{R}=\frac{[\text { anion }]_{\text {final }}\left|z^{-}\right|}{[\text {polymer }]_{\text {final }}\left|z^{+}\right|}
$$

\section{Equation 10: Ratio of anion to polymer}

\section{7: Chemical Exchange Saturation Transfer}

Chemical exchange saturation transfer (CEST) is a relatively new method for generating image contrast, which holds a great promise in in vivo imaging. Unlike $\mathrm{Gd}^{3+}$ based MRI contrast agents CEST agents require slow water exchange kinetics. CEST contrast is generated by saturating of a pool of solute protons that are in chemical exchange with bulk water protons but have a different chemical shift. Applying a low power radio frequency pulse at the resonance frequency of the solute exchangeable pool saturates these protons. Application of a radio frequency pulse $\mathrm{B}_{1}$ that is tuned to the frequency offset of the solute proton pool leads to a net excitation of protons in the lower energy 
states into their higher energy states-this is called saturation. The number of protons at each energy level will equalize and the proton pool will be saturated if the radio frequency pulse has enough energy as shown in scheme 2. The most common pathways of chemical exchange for CEST applications include proton molecular exchange, proton exchange, or a combination of both. An example of proton exchange is when a proton from an amide group moves to bulk water and an example of molecular exchange is when a water molecule moves between free water and a paramagnetic lanthanide metal associated with a paraCEST agents.

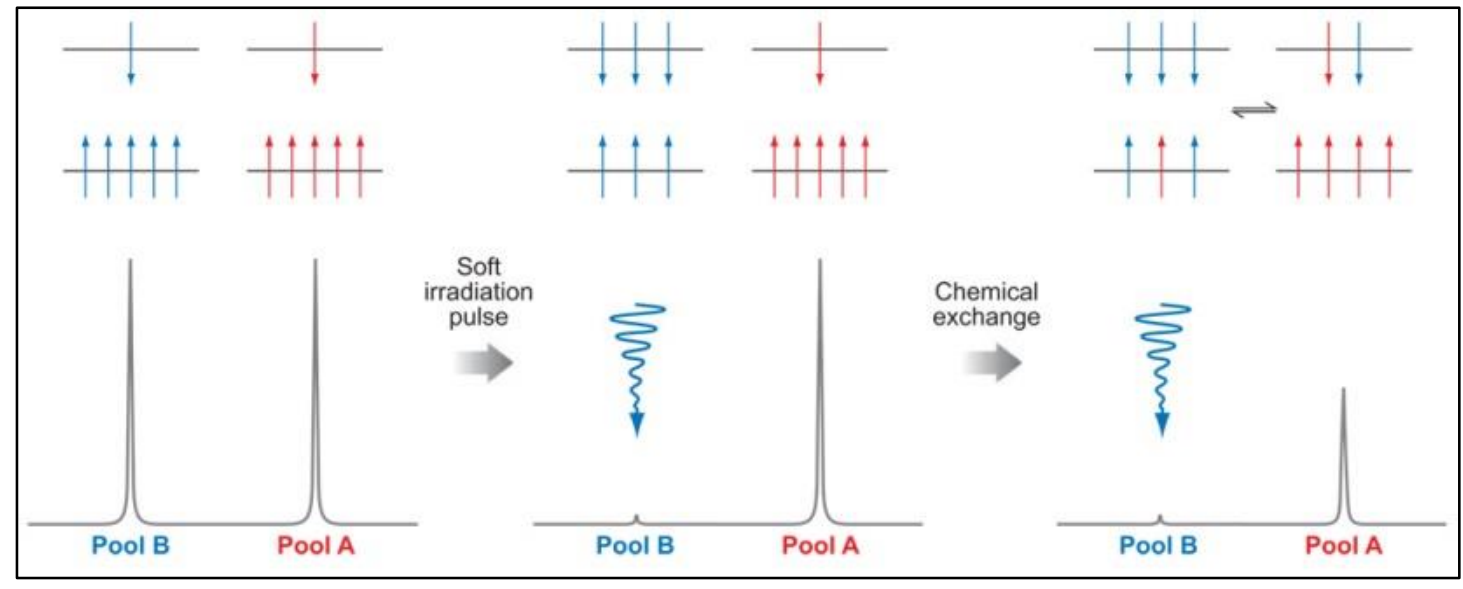

Scheme 2:Schematic representation of spins aligned with and against the field (upper and lower energy levels, respectively) above simulated NMR spectra (below) for two chemically distinct pools of nuclei (left), two spins after a saturation pulse has been applied $^{66}$

To observe CEST contrast chemical exchange should be slow to intermediate on the NMR time scale

$$
\Delta \omega \geq k_{\text {ex }}
$$

Equation 11: Exchange rate for CEST exchange rates 
Systems with longer $T_{l}$ values allow a larger range of viable exchange rates because the longer $T_{1}$ values allows the spins to maintain their magnetization longer. In paraCEST the shift is due to lanthanide ions and each lanthinde affords a different $\Delta \omega$. The large chemical shifts afforded by paramagnetic lanthanides allow faster exchange rates while mantaining the slow exchange regime between the bound and bulk water molecule. ${ }^{67}$ Although $\Delta \omega>2 \mathrm{ppm}$ can help avoid direct saturation of water ${ }^{68}$, larger values of $\Delta \omega$ can eliminate this problem completely.

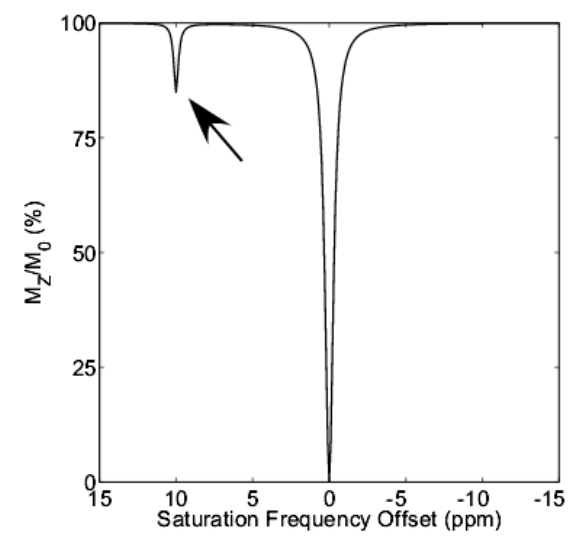

Figure1.12 : CEST spectrum; A CEST effect (or CEST peak) can be observed at $10 \mathrm{ppm}$ as highlighted with the arrow. The peak at 0 ppm is due to direct saturation of the bulk water protons by the $R F$ saturation pulse. ${ }^{59}$

A typical CEST MRI experiment consists of the acquisition of a series of MR images that are each preceded by an RF saturation pulse with an incrementally different frequency. The normalized MRI signal intensity is then plotted as function of the RF saturation pulse frequency offset relative to the bulk water resonance to create a CEST spectrum. If experimental parameters are appropriate then a reduction in MRI signal intensity referred to as a 'CEST peak' may be observed in the CEST spectrum. The form of a CEST spectrum is shown in Figure 1.12.the CEST peak at $10 \mathrm{ppm}$ is highlighted by an arrow. 
CEST enables detection of approximately $\mathrm{mM}$ proton pools that are in exchange with approximately $55 \mathrm{M}$ concentrations of water. The effects of chemical exchange saturation transfer have been investigated for more than 50 years but in the context of exogenous images agents for less than two decades. A modified version of the Bloch equations known as the Bloch-McConnell equation that included the CEST effect was introduced in 1957. The earliest example relating directly to CEST imaging was done by Forsen and Hoffman in 1963. They measured hydroxyl proton transfer rates between salicylaldehyde and water by placing a saturation pulse on resonance with solute protons and recording the solvent signal intensity inorder to study chemical exchange. It was Balaban and Ward in 2000 who coined the term CEST recently when they reported a new class of MRI contrast agents that were sensitive to their environment. They investigated applications of CEST agents to measure tissue temperature ${ }^{69}$ and $\mathrm{pH}^{70-72}$ Now there are many diverse CEST applications including cell labeling ${ }^{73}$, monitoring glucose uptake ${ }^{74}$ detection of the ischemic penumbra following stroke ${ }^{75}$, detection of zinc ions ${ }^{76}$ and many more.

\section{7.1: Diamagnetic CEST agents}

Initially molecules used to generate CEST were diamagnetic molecules known as diaCEST agents. DiaCEST was first reported using small molecules such as barbituric $\operatorname{acid}^{77}$ diaCEST agents can be either endogenous or exogenous molecules such as macromolecules, sugars, amino acids, glucosaminoglycans and heterocyclic compounds. The major drawback of diaCEST agents is the small size of their $\Delta \omega$. DiaCEST agents typically have $\Delta \omega<6 \mathrm{ppm}$ and this both limits the permitted rates of exchange as well as 
pre-saturation power (in order to avoid direct off-resonance saturation of water. Nonethelees diaCEST agents have been useful for exploring in vivo gene expression, imaging the in vivo distribution of glycogen ${ }^{74,} 78, \mathrm{pH}^{79,80}$ and studying cartilage degradation. ${ }^{81}$ The work on diaCEST has also been fundumental in discovering another class of contrast agents known as the paraCEST agents.

\subsection{2: Paramagnetic CEST agents}

Zhang and Sherry were the first to demonstrate that these paramagnetic chelates can be used for CEST imaging ${ }^{82}$. Initially paraCEST agents developed around paramagnetic lanthanide ions. Lanthanides vary along the $4 \mathrm{f}$ period and each metal has a different electronic structure and induce different hyperfine shift. The magnetic moment of the lanthnaide influences the magnitude of interaction between external magnetic field and magnetic moment. The magnetic susceptibility governs the lanthanide induced shifts (LIS). These large shifts make paraCEST agents more advantageous than diaCEST agents. These larger LISs $(\Delta \omega)$ allow faster exchange rates between the exchangable protons and bulk water without breaching the slow to intermediate exchange condition. This allows more spins to be transferred within a particular time frame, potentially increasing CEST. $T_{1}$ shortening contrast agents have at least one water molecule coordinated to the paramagnetic center but the rate of exchange for these complexes is much faster than the slow to intermediate exchange limit which is required for CEST agents. Slower exchange rates have been found for DOTA-tetraamide derivatives, such as DOTAM, where the acetate pendant arms have been replaced by acetamide arms. In comparison to carboxylates, amides are weaker electron donors and therefore create a 
more electron deficient lanthanide center, leading to a stronger water metal interaction. This ultimately slows water exchange. ${ }^{83}, 84$ The amide arms may also provide steric hinderance at the metal centre, this may also contribute to a slowing of the exchange of exchangable protons. The DOTAM ligand has been the elementary structure in the subsequent synthesis of paraCEST agents ${ }^{85}$. $\mathrm{Eu}^{3+}$ of all the lanthanide metals has the slowest rate of water exchange and a suitably low paramagnetic relaxation enhancement. These are favourable properties in the design of paraCEST agents, an example of such an agent is EuDOTAM-gly-Et. ${ }^{86}$ When $\mathrm{Eu}^{3+}$ is replaced with other lanthanide metals like $\mathrm{Tm}^{3+} \sim 50 p p m, \mathrm{Dy}^{3+} \sim 70 p p m, \mathrm{~Tb}^{3+} \sim 60 p p m$ and $\mathrm{Yb}^{3+} \sim 16 p p m$ and the chemical shift from the bulk water changes too, these values depends on the metal chosen. CEST MRI is relatively insensitive (even paraCEST) and often requires high concentration of contrast agents. The CEST efficiency is proportional to the number of exchangeable protons resonating at the same frequency per CEST molecule

\subsection{Enhaning sensitivity of CEST agents}

\subsection{1: Polymeric systems}

Increasing the number of exchangeable groups on the agent will make a CEST agent more sensitive. This approach to improve CEST sensitivity has been investigated by Pikkemaat ${ }^{87}$ and co-workers. They studied functionalized YbDOTAM ${ }^{3+}$ chelates with different generations of dendrimers of poly (propylene imine) and their applicability as $\mathrm{pH}$ responsive paraCEST agents. $\mathrm{Wu}$ and co-workers took a different approach preparing a linear polymer using free radical chain polymerization of EuDOTA-4AmCE 
derivatives. The detection limit in both studies were found to be orders of magnitude lower than the monomeric agents, but only linearly related to the number of metal ions. Thus, one viable approach to increasing the sensitivity of CEST is to increase exchanging sites associated with the agent.

\subsection{2: Optimization of the exchange rate}

One of the most important factors that affect CEST efficiency is the exchange rate. If the exchange rate is too fast the exchanging site cannot be activated and if the exchange rate is too slow there will be inefficient transfer of saturated spins, both of which will decrease the CEST effect. There is an optimal exchange rate for each exchanging site of a CEST agent and this optimal rate has been found to be dependent on the saturation power as well as the shift difference $\Delta \omega$. Higher saturation powers allows one to use fast water exchange rate, however for clinical application, the amount of power used is limited, and this limits the use of fast water exchanging systems. Therefore the design of CEST agents is focused on slower water exchange rates. Water exchange rate in paraCEST agents can be altered by altering the coordination geometry this depends on the type of isomer whether it is a square antiprism (SAP) or a twisted square antiprism $(\mathrm{TSAP})^{84,88}$ and ionic radii ${ }^{89,90}$, changing the lanthanide ${ }^{91}$ and the chemical environment

and the chemical environment is the polarity ${ }^{92,93}$ and the steric bulky of the side $\operatorname{arms}^{94}$. A lot of these parameters have been thoroughly explored in trying to optimize the water exchange rate of CEST agents; however, none afford the kinds of gains necessary to drive down detection limits to the extent needed for practical in vivo use. 


\subsection{3: Nano systems}

One strategy that has been used to optimize the CEST effect is nano systems, which have great promise owing to their high surface to volume ratio. One of the major strategies which have been used to increase the sensitivity of CEST agents is the encapsulation of a chemical shift reagent, e.g. TmDOTMA, inside a liposome. The encapsulated water molecule is then shifted away from the bulk water signal. Two water signals are observed on the NMR spectrum for these systems, the intra-liposomal water is shifted 3.1 ppm downfield from the bulk water. This intra-liposomal water gives rise to CEST because exchange across the phospholipid membrane is slow. Lanza and co-workers incorporated a bifunctional paraCEST agent on the surface of perfluorocarbon nanoparticles for molecular imaging; this increased the number of water exchanging sites. $^{95,96}$ Although, this system provides a large increase in payload and it does not compare with the liposome systems since the CEST effect again scales linearly with the number of chelates incorporated. 
Chapter 2. Nanoassembled Capsules:A Versatile Platform for developing molecular imaging agents

\section{1: Introduction}

Magnetic resonance imaging (MRI) has many attributes that make it ideal for molecular imaging. It has high spatial resolution and excellent soft tissue contrast and high resolution anatomical images can be obtained without radioactive tracers or ionizing radiation. The difference between the relaxation rate constants of normal and diseased tissues can be small and to generate contrast between these tissues contrast agents are sometimes used ${ }^{97,98}$. However, the relaxivity of the clinically available contrast agents is poor, normally $3-5 \mathrm{mM}^{-1} \mathrm{~s}^{-1}\left(25 \mathrm{MHz}, 25^{\circ} \mathrm{C}\right)^{20,99,100}$ and they are non-specific being delivered differently to tissue based on diffusion and vascular characteristics. This means that the applications of these contrast agents are limited. The low detectability arising from low relaxivity means that, high doses are often required of $\mathrm{Gd}^{3+}$ chelates $(0.1 \mathrm{mmol}$ $\left.\mathrm{kg}^{-1}\right)^{101}$. This poses a problem for visualization of specific biomarkers of disease by molecular imaging, since these biomarkers are present at much lower concentrations usually in the micromolar range. Hence increasing the detectability of MRI contrast agents is the key to developing MR based molecular imaging applications. This can be achieved by increasing the relaxivity of the contrast agents by optimizing structural and dynamic properties or by increasing the accumulation of contrast agents to the site of interest. Nano assembled capsules (NACs) are one way of achieving both of these goals. Wong and co-workers developed a nano encapsulation method in which negatively charged nanoparticles, cationic polymers and multivalent anions are combined to

generate silica/polymer nanocapsules. ${ }^{64,102,103}$ The synthesis of these nanoparticles is a 
two step method where a cationic polymer and a polyanion ${ }^{104}$ are added together in solution to form an aggregate. An aqueous suspension of $\mathrm{SiO}_{2} \mathrm{NPs}$ is added to form a shell around the aggregate.

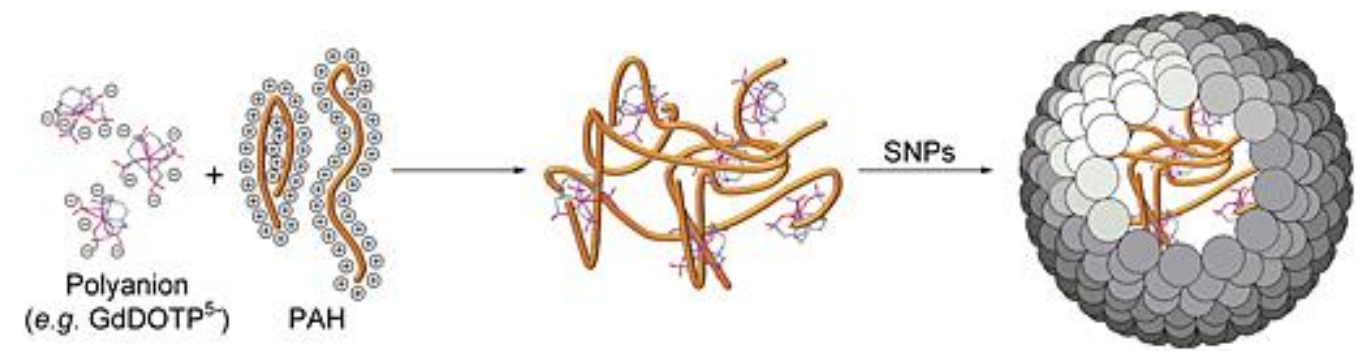

Scheme 3: A Schematic representation of the preparation of nano assembled capsules which are $\mathrm{SiO}_{2}-\mathrm{NP}$-coated

The work of Wong and co-workers was extended to the development of contrast agents by Plush et al.: NACs were prepared using the polyanionic chelate GdDOTP ${ }^{5-}$ and pollyallyl amine hydrochloride (PAH) as the cationic polymer, followed by encapsulation with $\mathrm{SiO}_{2} \mathrm{NPs}^{62}$. The encapsulation of $\mathrm{GdDOTP}^{5-}$ was found to afford significant increases in relaxivity and various synthetic parameters were found to offer good control over the size of the NACs produced. GdDOTP ${ }^{5-}$ relaxivity arises mainly from the second sphere of water molecules formed through hydrogen bond interaction between the phosphates and the water molecules from the bulk water. ${ }^{105-107}$ Phosphonates are better second hydration sphere generators compared to carboxylates. For GdTTHA ${ }^{3-}$ the contribution is entirely from the outer hydration sphere as a free chelate. Even though all four chelates do have some outer hydration sphere contribution of about $1.8 \mathrm{mM}^{-1} \mathrm{~s}^{-1}(20$ $\left.\mathrm{MHz}, 25^{\circ} \mathrm{C}\right)^{107,} 108$ to the overall observed relaxivity of the chelates. GdDOTA ${ }^{1-}$ and GdDTPA $^{2-}$ are inner sphere chelates and their primary relaxivity contribution arises from 
the inner hydration sphere, where the relaxivity arises mainly from the exchange of the inner hydration sphere water molecule with the bulk water. The inner sphere contribution to relaxivity is approximately $2.9 \mathrm{mM}^{-1} \mathrm{~s}^{-1}\left(20 \mathrm{MHz}, 25^{\circ} \mathrm{C}\right){ }^{109}$ hence as free chelate GdTTHA $^{3-}$ has a very low relaxivity compared to the other carboxylates. NAC synthesis was performed using the synthesis method which was previously reported ${ }^{62}$.
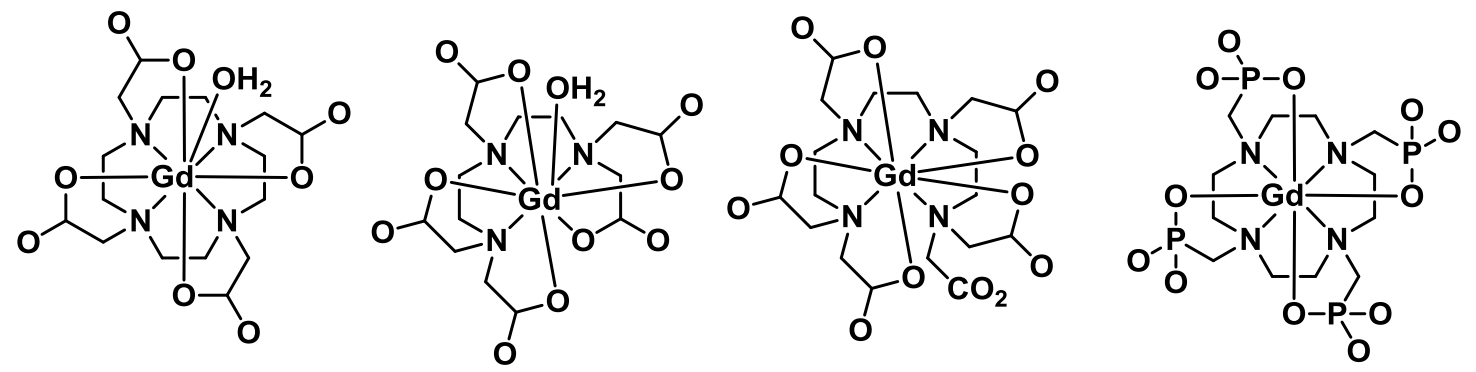

Scheme 4: The structures of the chelates investigated in this work ${ }^{16} \mathrm{GdDOTA}^{1-}$, GdDTPA ${ }^{2-}, G_{T T T H}^{3-}$, and GdDOTP ${ }^{5-}$

Although these results are encouraging, it was unclear to what extent this encapsulation strategy can be applied to other chelates, in particular those with charges lower than 5-. To do so we investigated the encapsulation with the following chelates GdDOTA ${ }^{1-}$, GdDTPA $^{2-}$, GdTTHA ${ }^{3-}$, GdDOTP ${ }^{5-}$ which are, monoionic, diionic, triionic, and pentaionic chelates, respectively ${ }^{62}$. This choice of chelates also allows another effect to be studied: the effect of encapsulating chelates with different hydration states. GdDOTP $^{5-}$ chelate which does not contain a coordinated water molecule $(q=0)$, the chelates GdDOTA ${ }^{1-}$ and GdDTPA ${ }^{2-}$ are $q=1$ and this could afford higher relaxivity. The idea was to use these negatively charged chelates as salts, to form aggregates with a positively charged polymer (PAH) upon mixing and coat this aggregate with silica 
nanopaticles as the shell material. Formation of Gd-NACs through self assembly is shown in Scheme 3.

\section{2: Experimental}

\subsection{1: Materials}

Poly(allylamine hydrochoride),(PAH, 56 000g/mol) 596 allylamine units per molecule were purchased at Sigma Aldrich, collodial $\mathrm{SiO}_{2} \mathrm{NPs}$ was purchased at Nissan chemicals and $\mathrm{H}_{8}$ DOTP was purchased from macrocyclics Dallas,TX,USA. GdDOTA ${ }^{1-}$, GdDTPA $^{2-}$ GdTTHA $^{3-}$ and GdDOTP ${ }^{5-108,110,111}$ were prepared as previously published. ${ }^{112,}$

113 The concentration of the chelates was adjusted to a $\mathrm{pH}$ of 9 with $1 \mathrm{M} \mathrm{NaOH}$. The $\mathrm{SiO}_{2} \mathrm{NPs}$ solution was diluted to a concentration of $2 w t \%$ by adding $1 \mathrm{~mL}$ of the $20.3 w t$ $\%$ collodial solution in water to $9 \mathrm{~mL}$ of acetonitrile.

\subsection{2: Synthesis of Nanoassembled capsules}

A $\left(3 / 2 \mathrm{MeCN} / \mathrm{H}_{2} \mathrm{O}\right) \mathrm{PAH}$ solution $(20 \mu \mathrm{L}, 5 \mathrm{mg} / \mathrm{mL})$ was mixed with a $\left(3 / 2 \mathrm{MeCN} / \mathrm{H}_{2} \mathrm{O}\right)$ $\mathrm{Gd}^{3+}$ solution $(120 \mu \mathrm{L})$ in an eppendorf tube and was vortexed at a low speed. A turbid solution immediately resulted indicating the formation of a polymer-chelate aggregate; the aggregates were then aged for 10 minutes. Diluted $\mathrm{SiO}_{2} \mathrm{NP}$ suspension $(2 w t \%$ in $\mathrm{MeCN}$ ) was added,and the resultant mixture was vortexed at a medium speed. $R$ charge ratios of $0.3,0.5,0.75,1.0,1.25$ were studied in this work. It is defined as the ratio of total number of negative charges from the $\mathrm{Gd}^{3+}$ chelates to the total number of positive charges from the polymer as shown in equation $2.1^{114}$. 


$$
R=\frac{[\text { anion }]_{\text {final }}\left|z^{-}\right|}{[\text {polymer }]_{\text {final }}\left|z^{+}\right|}
$$

A turbid solution immediately resulted, indicating the formation of polymer-anion aggregate. The aggregates were aged for 10 minutes before the $\mathrm{SiO}_{2} \mathrm{NPs}$ suspension was added. The resultant mixture was vortexed at a medium speed. The precipitate was recovered through centrifugation.

\section{3: Characterization}

\subsection{1: Dynamic light Scattering}

Hydrodynamic light scattering was carried out with Horiba (Irvine,CA,USA) LB550 dynamic light scattering instrument. It has an autocorrelator at a laser wavelength of 650 nm. All studies were carried out at a $90^{\circ}$ scattering angle, and were temperature controlled at $25^{\circ} \mathrm{C}$ in polystyrene cuvvetes. Freshly syringed samples were dispersed in water and measured at four dilutions to ensure size distributions were independent of concentration effects. Samples were regularly agitated to prevent settling of larger particles.

\subsection{2: Scanning Electron Microscopy}

SEM was carried out using a FEI, equipped with secondary electron, X-ray (EDX) detectors. Secondary electron images were taken at $5 \mathrm{kV}$ with a working distance of 5-10

mm. A droplet of NACs suspension was placed on the aluminum stub and dried in air. The sample was then sputter coated with gold for 55 seconds. 


\subsection{3: Relaxometry}

Longitudinal water proton relaxation $T_{l}$ were measured on a $0.47 \mathrm{~T}$ Bruker Biospin (Billerica, MA, USA) MiniSpec contrast agent analyzer operating at $19.99 \mathrm{MHz}$ using an inversion recovery pulse sequence. NACs were suspended in water $(1 \mathrm{~mL})$ at $\mathrm{Gd}^{3+}$ concentrations ranging from $0.22 \mathrm{mM}$ to $2.37 \mathrm{mM}$. Samples were regularly agitated to guard against settling of larger particles. Relaxivity values were determined in Excel by linear regression analysis of the experimentally determined $R_{1}$ values as a function of $\mathrm{Gd}^{3+}$ concentration.

\subsection{4: ICP-OES Gd ${ }^{3+}$ concentration determinations}

Concentration determination of Gd was performed using a Perkin Elmer (Waltham, MA, USA) Optima 2000 inductively coupled plasma optical emission spectrometer (ICPOES). $\mathrm{Gd}^{3+}$ standards were produced by quantitative serial dilution of a commercial 1000 $\mathrm{mgL}^{-1}$ gadolinium in $2 \%$ nitric acid standard (Fluka Analytical) into a $0.1 \%$ nitric acid solution. Samples were prepared by digesting the dried samples with $70 \% \mathrm{HNO}_{3}$ $(100 \mu \mathrm{L})$ and water $(900 \mu \mathrm{L})$. The resulting analyte was vortexed thoroughly, and then filtered to remove any particulate using a $0.2 \mu \mathrm{m}$ filter to produce the final sample for ICP-OES analysis. Sample analyte concentrations were calculated to fall in the middle of the constructed ICP-OES $\mathrm{Gd}^{3+}$ calibration curve to ensuring, accurate $\mathrm{Gd}$ spectral readings. Readings were taken in triplicate and averaged. The highest percent relative standard deviation allowed between these replicates was $1 \%$, to ensure precise $\mathrm{Gd}^{3+}$ spectral readings for the samples analyzed in this work. The concentration of $\mathrm{Gd}^{3+}$ was determined for each sample and then multiplied by the dilution factor. 


\section{4: Results and Discussion}

The size of the NAC and per $\mathrm{Gd}^{3+}$ relaxivity were found to be inversely related. For the benefit of our research it was desirable to keep certain synthetic variables, such as solvent and cationic polymer, fixed. The effect of varying the polymer used for aggregate formation has already been explored by Plush and co-workers. ${ }^{62}$ The nature of the polymer was found to have an effect on the size of the NACs attained and polly allyl hydrochloride amine was found to give smaller NACs compared to the other polymers studied. Smaller NACs afforded higher per $\mathrm{Gd}^{3+}$ relaxivities, therefore for this work only PAH was used for NAC synthesis. In all experiments in this chapter the solvent mixture used was $3: 2 v / v$ aetonitrile and water mixture. Stock solutions of the chelate $\mathrm{Gd}^{3+}$ and polymer were prepared in 3:2 v/v of acetonitrile and water. In all cases, upon addition of the anionic chelate the solution became turbid except for cases in which the anion was GdDOTA $^{1-}$. The reaction were stirred vigorously for 15 seconds, the aggregate was aged for ten minutes, and then $\mathrm{SiO}_{2}$ nanoparticles in $100 \%$ acetonitrile was added. The addition of $\mathrm{SiO}_{2}$ nanoparticles as the aggregate coating was crucial because the $\mathrm{SiO}_{2}$ nanoparticles stabilize the polymer chelate aggregate. To ensure that there was no distortion on the relaxivity measurements by any non-encapsulated $\mathrm{Gd}^{3+}$ chelates the NACs were put through a series of centrifuge cycle washes in $10 \mathrm{kDa}$ MWCO centrifugal filter units. The constitution of the $2 w t \% \mathrm{SiO}_{2}$ nanoparticles stock suspension was found to be critical to the formation of NACs; if the $\mathrm{SiO}_{2}$ nanoparticles were suspended in water alone, or a 3:2 v/v MeCN/ $\mathrm{H}_{2} \mathrm{O}$ mixture, then NAC formation was not observed. NACs were characterized using dynamic light scattering for size analysis. It was 
observed that as the charge ratio $R$ increased the average size of the NACs was observed to increase independent of the chelate being studied.

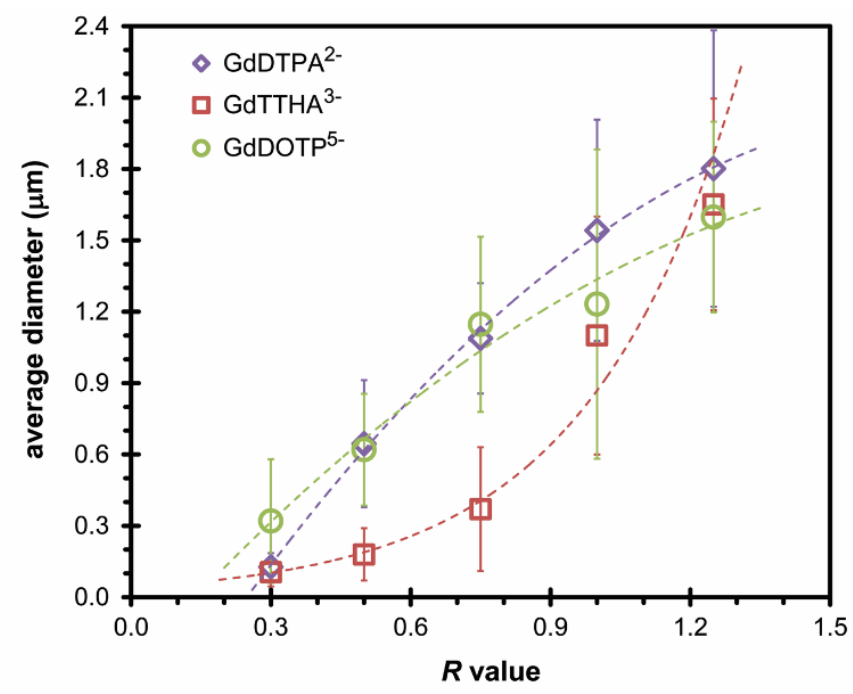

Figure 2.13: The effect of changing the $R$ value on the average hydrodynamic diameter of the NACs produced for GdDTPA ${ }^{2-}$ (purple), GdTTHA ${ }^{3-}$ (red) and GdDOTP ${ }^{5-}$ (green). Dashed lines are a guide to the eye only; error bars represent the $1^{{ }^{s t}}$ standard deviation of the size distribution of the capsules. Sizes and their distributions are the average of 6 NAC preparations ${ }^{115}$

There was no general relationship between the size of the NACs and the charge ratio observed across all the three chelates studied. The size of NACs encapsulating GdDTPA $^{2-}$ and GdDOTP ${ }^{5-}$ was observed to increase sublinearly with increasing $R$ value even though structural relationship is not identical between these two chelates. In contrast, for NACs encapsulating GdTTHA ${ }^{3-}$ the size of NACs seemed to increase superlinearly with increasing R (Figure 2.13). On a structural basis, this observation is difficult to explain. The only notable difference in structure is that GdTTHA ${ }^{3-}$ unlike GdDTPA $^{2-}$ and GdDOTP ${ }^{5-}$ it has one uncoordinated carboxylate. So, one possible explanation is that while the charge on GdDTPA ${ }^{2-}$ and GdDOTP $^{5-}$ is well distributed on 
the chelate, part of the charge on GdTTHA ${ }^{3-}$ is concentrated on one of the carboxylates; this may alter the interaction of the chelate and the cationic polymer..
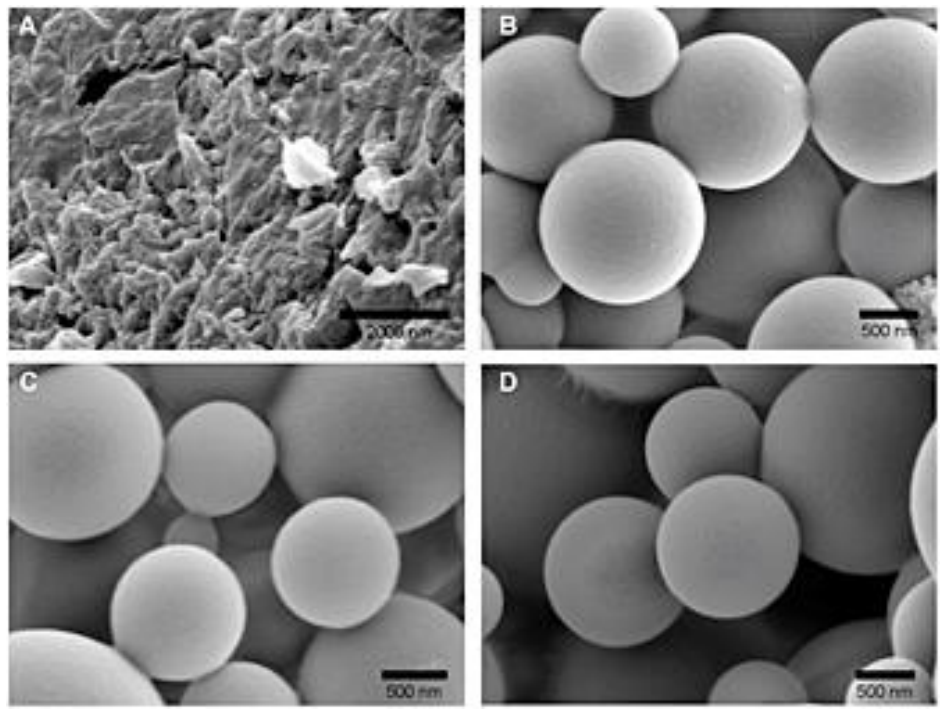

Figure 2.14: SEM images of NAC preparations in 3:2 v/v MeCN/H $\mathrm{H}_{2} \mathrm{O}$ with $\mathrm{PAH}$ and: A) $\left.G d D O T A^{1-} R=1.0 ; B\right) G d D T P A^{2-} R=0.75$; C) GdTTHA $\left.{ }^{3-} R=0.75 ; D\right) G d D O T P^{5-} R=$ 0.75

The production of NACs was apparent from the SEM images on Figure 2.14 of capsules which have been shown to withstand several rounds of centrifuge without losing its structural integrity. GdDTPA ${ }^{2-}$, GdTTHA ${ }^{3-}$ and GdDOTP $^{5-}$ are capable of forming aggregates with the polymer in a way that will allow NAC formation. Furthermore, the monoionic GdDOTA ${ }^{1-}$ appears incapable of aggregation in a manner that allows NAC formation. It was shown by Wong and co-workers that monovalent acetate could not ionically cross link polymer chains, this was confirmed by Monte Carlo simulations, where higher valent salt caused polymer to be in a collapsed or condensed form ${ }^{114}, 116$ hence increasing the ability of the polymer to cross link with the multivalent anion. The 
incorporation extent of $\mathrm{Gd}^{3+}$ chelate was determined using ICP-OES were the NACs were digested using 15.7 $\mathrm{M}$ nitric acid. The concentration of $\mathrm{Gd}^{3+}$ was measured in both the supernatant and the NAC. Overall there was greater than $99 \%$ incorporation of the $\mathrm{Gd}^{3+}$ chelates achieved for NACs from all the chelates, though a lower percentage value was observed for chelates like GdDTPA ${ }^{2-}$ which had $>93 \%$ incooperation as shown in table 2 , For GdDOTA ${ }^{1-}$ no further studies were performed because there was no consistency in the formation of NACs. The per $\mathrm{Gd}^{3+}$ relaxivity was calculated using the regression analysis by plotting the longitundal relaxation rate constant (determined by inversion recovery) against the concentration of the $\mathrm{Gd}^{3+}$ : the gradient is the relaxivity. The effect of changing the $R$ value on the per $\mathrm{Gd}^{3+}$ relaxivity is shown in Figure 2.15. These results are braodly similar to those observed previously with GdDOTP ${ }^{5-}$ and PAH NACs in a different solvent mixture: as the $R$ value is increased the per-Gd ${ }^{3+}$ relaxivity decreases. In all cases the smaller the NAC size the higher the per $\mathrm{Gd}^{3+}$ relaxivity observed..$^{62}$

In comparison the GdDTPA ${ }^{2-}$ continaing NACs have a lower relaxivity of the same $R$ value (0.3), even though it is a $q=1$ chelate. Hence, it seems that the contribution of the inner hydration sphere seem to be masked upon encapsulation. Encapsulation of $q=1$ chelate did not seem to have an effect on relaxivity as was suggested in comparison to the effect it had on $\mathrm{q}=0$ chelates and GdDOTA $^{1-}$ of a lower valence 1 could not afford to form aggregates with the polymer therefore no further studies were perfomed on GdDOTA $^{1-}$. Encapsulation of chelates do have an effect on the overall relaxivity attained by these chelates in comparison to the relaxivity of free chelates, NACs had higher 
relaxivity values even for larger capsules which had lower relaxivity values, they still attained higher or equal relaxivity values compared to the free chelates as shown in Fig 2.13 .

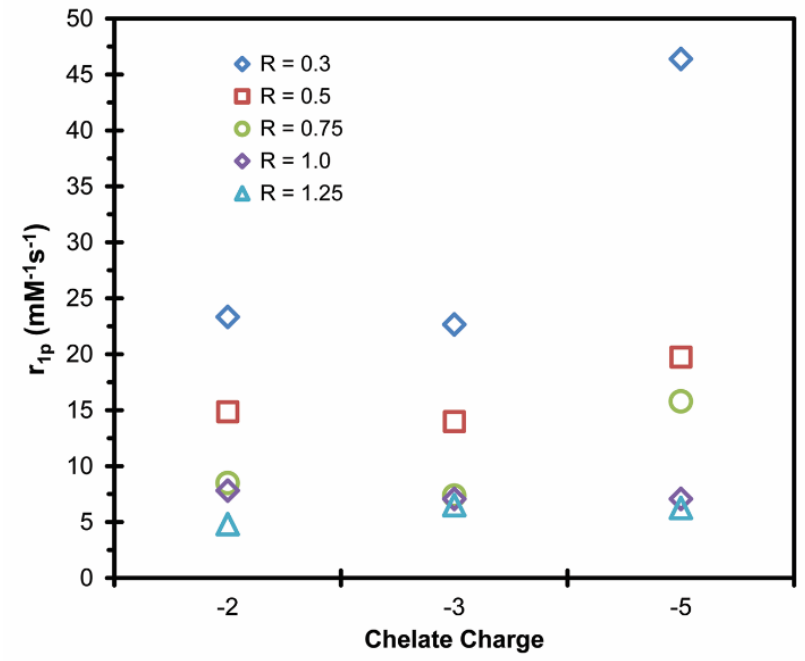

Figure 2.15: The effect of changes in the overall Gd3+ chelate charge on the relaxivity of the NACs produced for different $R$ values

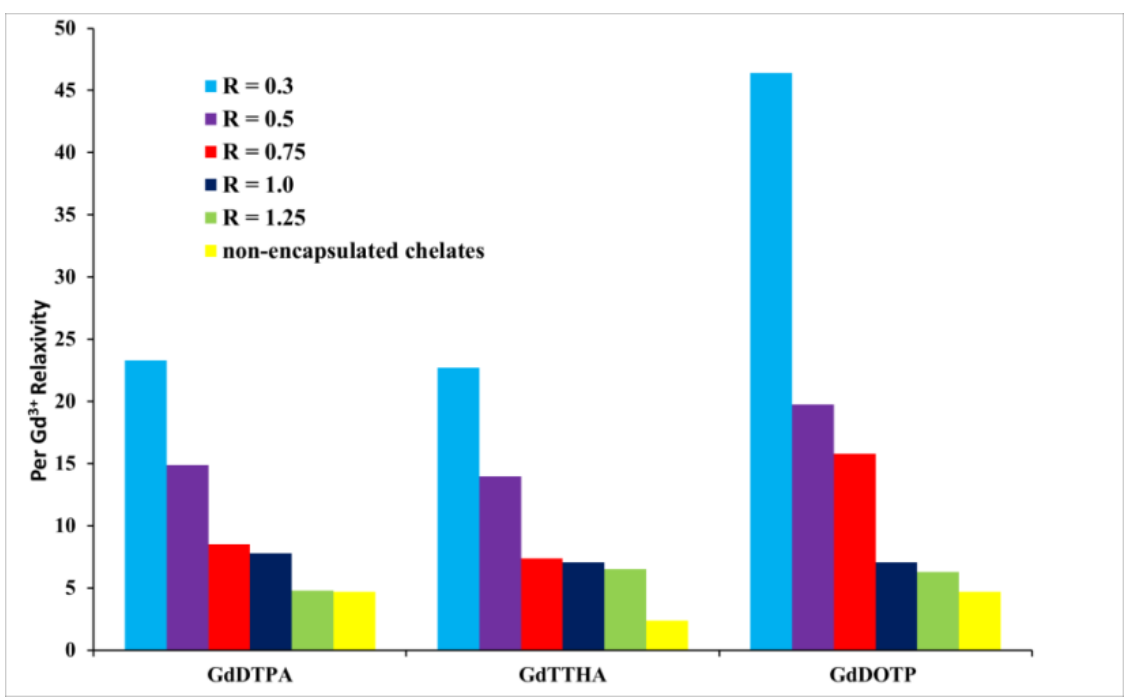

Figure 2.16: Relaxivity values measured at $0.47 \mathrm{~T}$ at $25^{\circ} \mathrm{C}$ of encapsulated $\mathrm{Gd}$ chelates compared to the relaxivity values of the free chelates 
Table 2: Summary of the variations in size and relaxivity arising from changing the chelate charge and $R$ value during the preparation of NACs.

\begin{tabular}{|c|c|c|c|c|}
\hline Chelate & $\mathbf{R}$ value & $\% \mathbf{G d}^{3+}$ incorp $^{\mathrm{a}}$ & Diameter $\mathbf{n m}^{\mathbf{b}}$ & $r_{1} \mathrm{mM}^{-1} \mathrm{~s}^{-1 \mathrm{c}}$ \\
\hline \multirow[t]{5}{*}{ GdDTPA $^{2-}$} & 0.30 & 99.7 & $127 \pm 58$ & $23.3 \pm 0.3$ \\
\hline & 0.5 & 94.5 & $645 \pm 267$ & $14.9 \pm 0.1$ \\
\hline & 0.75 & 93.3 & $1088 \pm 232$ & $8.5 \pm 0.05$ \\
\hline & 1.0 & 99.0 & $1542 \pm 465$ & $7.8 \pm 0.01$ \\
\hline & 1.25 & 99.9 & $1802 \pm 581$ & $4.8 \pm 0.1$ \\
\hline \multirow[t]{5}{*}{ GdTTHA $^{3-}$} & 0.3 & 99.6 & $105 \pm 60$ & $22.7 \pm 0.1$ \\
\hline & 0.5 & 99.9 & $180 \pm 110$ & $14.0 \pm 0.1$ \\
\hline & 0.75 & 99.9 & $370 \pm 260$ & $7.4 \pm 0.3$ \\
\hline & 1.0 & 99.0 & $1100 \pm 500$ & $7.1 \pm 0.3$ \\
\hline & 1.25 & 99.9 & $1651 \pm 445$ & $6.5 \pm 0.1$ \\
\hline \multirow[t]{5}{*}{ GdDOTP5 $^{5-}$} & 0.3 & 99.9 & $320 \pm 260$ & $46.4 \pm 0.3$ \\
\hline & 0.5 & 99.1 & $620 \pm 235$ & $19.8 \pm 0.1$ \\
\hline & 0.75 & 98.9 & $1147 \pm 368$ & $15.8 \pm 0.1$ \\
\hline & 1.0 & 98.6 & $1232 \pm 650$ & $7.1 \pm 0.1$ \\
\hline & 1.25 & 99.5 & $1598 \pm 401$ & $6.3 \pm 0.2$ \\
\hline
\end{tabular}

1. ${ }^{a}$ The percentage of $\mathrm{Gd}^{3+}$ chelate added to the NAC synthesis that was found to be incorporated into the NAC.

2. ${ }^{b}$ The mean average hydrodynamic diameter of 6 NAC preparations \pm the $1^{\text {st }}$ standard deviation of the size distribution, determined by dynamic light scattering.

3. ${ }^{c}$ The mean longitudinal relaxivity of $6 \mathrm{NAC}$ preparations \pm the $1^{\text {st }}$ standard deviation, determined at $20 \mathrm{MHz}$ and $25^{\circ} \mathrm{C} .{ }^{115}$ 
The relaxivity attained using highest $R$ charge ratios, which produced large NACs, was much lower and was independent of the chelate used to synthesize the NACs. In comparison to the NACs containing carboxylates chelates $\left(\right.$ GdDTPA $^{2-}$ and GdTTHA $\left.{ }^{3-}\right)$, the NACs containing GdDOTP ${ }^{5-}$ had a substantially higher relaxivity compared to the slightly smaller NACs produced from the carboxylates containing chelates. This is probably due to the known promotion of the strong second sphere interaction of the water molecules with the water molecules from the bulk water and the phosphonate groups of GdDOTP $^{5-}$ more than the carboxylates groups this tend to compensate the contribution from the inner hydration sphere hence GdDOTP ${ }^{5-}$ attained higher relaxivity. Encapsulation has effectively reduced the molecular tumbling of these chelates and the water molecules tend to be bound in the second sphere increasing water exchange rate and reducing $r_{G d H}$. Increasing the distance of the water molecule to the metal centre from the inner hydration sphere has a major effect on relaxivity given that the rotational correlation time is reduced. Upon encapsulation of the carboxylate chelates GdDTPA ${ }^{2-}$ the exchange of inner sphere water molecules is extremely slow or the exchange is so insignificant that its contribution to overall relaxivity attained is not observed, hence the overall contribution of the inner hydration sphere relaxivity on GdDTPA ${ }^{2-}$ does not show in its relaxivity which is similar to that of GdTTHA ${ }^{3-}$ a chelate without a coordinated water molecule. For these reasons encapsulation has a greater effect on the relaxivity of GdTTHA $^{3-}$ than that of GdDTPA ${ }^{2-}$. GdTTHA ${ }^{3-}$ has a relaxivity of just $2.65 \mathrm{mM}^{-1} \mathrm{~s}^{-1}$ as a free chelate arising primarily from an outer sphere contribution, ${ }^{35}$ but showed approximately 8.6 times increase in relaxivity for $R=0.3$ NACs (Figure 2.16). This 
increase in relaxivity of GdTTHA ${ }^{3-}$ could be attributed to a magnification of the second sphere effect upon encapsulation of GdTTHA ${ }^{3-}$ therefore an abrupt increase in relaxivity was observed for this chelate relaxivity. We speculate that inside the NAC water is comparativiely scarce and therefore water in the inner hydration sphere of GdDTPA ${ }^{2-}$ is unable to freely exchange and this mechanism contributes insignificantly to the overall relaxivity. Proton exchange ${ }^{113}$ may be a more important mechanism for the transfer of relaxation from the chelate to the bulk rather than the whole water molecule diffusing between the bulk water and NAC interior.

\section{5: Conclusions}

In conclusion, encapsulation of $\mathrm{Gd}^{3+}$ chelates is a robust and versatile method to synthesize high payloads of contrast agents, and attain very high relaxivity values without expensive modification to the contrast agents. Lower charge densities like 2- were well tolerated in the synthesis of NACs, they formed capsules for all the $R$ values studied in this chapter but charge of -1 could not form a consistency formation of capsules. As was shown by Wong and co-workers that monovalency acetate could not ionically cross link polymer chains, this was confirmed by Monte Carlo simulations, where higher valent salt caused polymer to be in a collapsed form hence better interaction with the polymer. Therefore a dependence on charge was observed the lower the charge of the chelate the less the interaction of the chelate with the polymer because for lower charge density, less turbid solution were observed for the chelate GdDTPA ${ }^{2-}$ compared to the turbidity observed for GdDOTP ${ }^{5-}$ of the same charge ratio, therefore charge do have an effect on the formation of the aggregate. The ability of a chelate to form an aggregate with the 
polymer has a profound effect on the rigidity attained to reduce molecular tumbling on the chelate hence higher charge density tend to have a better interaction with the polymer hence better rigidity attained by higher charges compared to lower charges as was shown by the relaxivity values attained by smaller capsules which have better interaction with the polymer on forming the aggregate hence better reduction in molecular tumbling in solution of the small chelates. Size of the NACs was affected by the $R$ value and cation employed, there was a general trend that the smaller the $R$ value the smaller the size of the NACs produced. Encapsulation of $\mathrm{GdDTPA}^{2-}$ a $q=1$ chelate did not show to enhance the relaxivity attained by this chelate, GdDOTP ${ }^{5-}$ and GdTTHA $^{3-} q=0$ chelates had very competetive relaxivity values, which we speculated that this could be that upon encapsulation the contribution from the inner hydration sphere becomes scarce therefore its effect is not observed in the per $\mathrm{Gd}^{3+}$ relaxivity attained from the $q=1$ chelates. In this work we observed that the smaller the NAC the better the restriction in tumbling of the chelates hence higher per $\mathrm{Gd}^{3+}$ relaxivity for smaller NACs compared to their larger counterparts. Encapsulation of $\mathrm{Gd}^{3+}$ chelates allows high payload delivery of these chelates and therefore an increase in relaxivity especially for targeted MR imaging where $\mathrm{T}_{2}$-shortening iron oxide contrast agents are used at lower concentrations. This work shows that encapsulation of $\mathrm{Gd}^{3+}$ chelates increases relaxivity values by reducing molecular tumbling and can also deliver high payloads of the imaging agent this may decrease limits of detection to levels at which biomarkers for disease pathology exist. . In addition to slowing the tumbling of the chelates by forming an aggregate with the polymer, when designing a contrast agent for MRI one must ensure that water molecules associated with the NAC have access to bulk water as this is critical to the function of an 
MRI contrast agent. For this reason making smaller NACs is crucial for achieving the highest per- $\mathrm{Gd}^{3+}$ relaxivity. In these smaller NACs encapsulation allows sufficient movement of water protons to allow an enhancement in relaxivity for all chelates. 
Chapter 3. An Alternative Coating Method for the High Payload Synthesis of Nano Capsule MRI Contrast Agents

\subsubsection{Introduction}

We have demonstrated that NACs represent a versatile and robust platform that may allow the dvelopment of targeted MRI contrast agents for molecular imaging applications with very low detection limits. However, concern must remain about the in vivo stability of these capsules with their shells that are held togather solely by electrostatic forces. One possible solution to this problem may be the use of capsules that also contain a high payload of a $\mathrm{Gd}^{3+}$ chelate encapsulated within a covalently linked shell. Capsules should incorporate as many contrast agents as possible into a single capsule to facilitate the delivery of large payloads to a pathology of interest in targeted imaging applications. Stucky and co-workers reported the same synthesis approach. ${ }^{117}$ These spheres are called nano-assembled capsules (NACs). The synthesis of NACs is rapid and it occurs under

ambient conditions at mild $\mathrm{pH}$ values. ${ }^{62}$ Synthesis method of NACs have been used to encapsulate a clinical florescent probe indocyane green ${ }^{102}$ and $\operatorname{GdDOTP}^{5-62}$ an $\mathrm{MR}$ contrast agent and had a significant enhancement on relaxivity of GdDOTP ${ }^{5-}$. In another work we published a series of $\mathrm{Gd}^{3+}$ chelates where encapsulated in these capsules and there was a significant increase in relaxivity of these chelates, attaining relaxivity value of $46.3 \mathrm{mM}^{-1} \mathrm{~s}^{-1}$ for chelates of $\mathrm{GdDOTP}^{5-} R=0.3$ which is ten times more than the relaxivity value of the free chelate. ${ }^{115}$ The NAC synthesis method has advantages compared to other synthetic methods of increasing a high payload of metal centres because it is performed at room temperature and is synthetically straight forward. The 
polyanionic $\mathrm{Gd}^{3+}$ chelates are mixed with a solution of a polymer in a mixture of a organic/aqueous solution and forms an aggregate which is encapsulated with an $\mathrm{SiO}_{2} \mathrm{NPs}$ to form a NAC. The formation of NACs is driven by the electrostatic attraction of the ions involed. Due to this ectrostatic attraction this tends to raise questions on how robust these NACs are in vivo, hence need to devise another route to synthesize an alternative coating method. A new encapsulation method was employed which uses peptidic linking to form the capsule shell. Herein we describe the development of Crosslinked Assembled Capsules (CACs) that are prepared firstly by mixing the polymer solution and a poly anion to form an aggregate in the same manner as for NACs. The surface of the aggregate is covered with primary amines which could be used to form peptidic bonds with a dicarboxylic acid, such as succinic acid,to coat and crosslink the surface with a peptide coupling reagent (EDC). This encapsulation method used was investigated with a view to produce CACs encapsulating GdDOTP ${ }^{5-}$. The initial stage of synthesis of this work was performed using PAH and pyrophosphate solutions and glutamic acid was used to coat the aggregate and EDC for cross linking.

\subsubsection{Experimental}

Water refers to deionized water with a resistivity of $18.2 \mathrm{M} \Omega$. All solvents and reagents were purchased from commercial sources and used as received unless otherwise stated. Pollyallylamine hydrochloride (PAH) (56,000 MW), poly-L-lysine (50,000 MW), Lglutamic acid, succinic acid and 1-ethyl-3-(3-dimethylaminopropyl) carbodiimide (EDC) were purchased from the Sigma-Aldrich corporation and used as received. $\mathrm{H}_{8}$ DOTP was 
purchased from Macrocyclics. GdDOTP ${ }^{5-}$ was synthesized by previously reported methods. ${ }^{108,110,118}$.

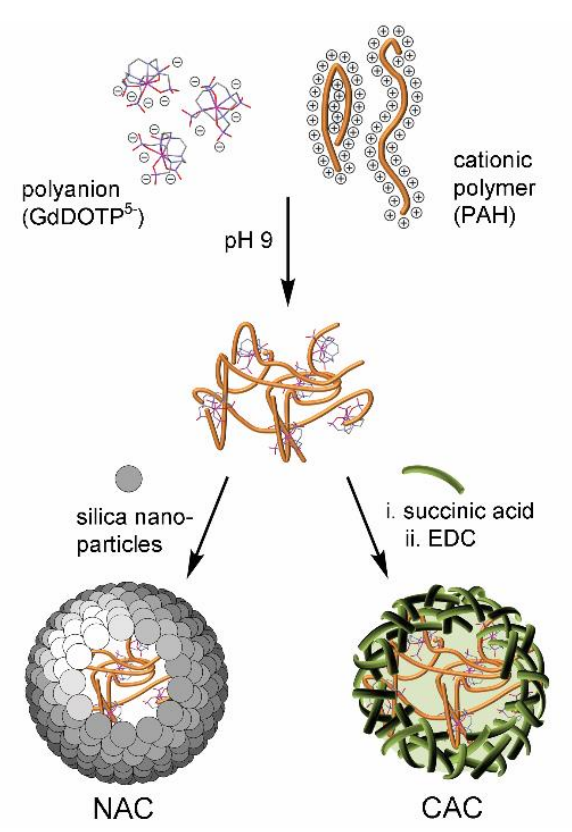

Scheme 5: Schematic of synthesis of cross linked assembled capsules versus that of NACs

\subsubsection{Synthesis of CACs with Simple Diamagnetic Anions}

Stock solutions of polymer and anion were prepared in $100 \%$ water at $\mathrm{pH} 9$. Stock solution concentrations were as follows: $($ polymer $)=89 \mu \mathrm{M}$, (citrate $)=4.2$ to $19.9 \mathrm{M}$ and $($ pyrophosphate $)=2.9$ to $14.9 \mathrm{M}$. The polymer $(20 \mu \mathrm{L})$ and anion $(120 \mu \mathrm{L})$ stock solutions were then added to water $(10 \mathrm{~mL})$ at $\mathrm{pH} 9$. The charge ratio $R$ was varied by varying the initial concentration of anion. After mixing the reaction immediately became turbid reflecting the formation of a polymer-anion aggregate. The solution was vortexed at low speed for $10 \mathrm{~s}$ and then aged for 3 minutes without agitation. After aging, a solution of dicarboxylic acid (20 $\mu \mathrm{L}, 0.089 \mathrm{M})$ was added. Succinic acid or L-glutamic 
acid were used at identical concentrations. The solution was gently mixed and then aged for 3 minutes. A solution of 1-ethyl-3-(3-dimethylaminopropyl carbodiimide $(20 \mu$, $0.174 \mathrm{M}$ ) in $\mathrm{H}_{2} \mathrm{O}$ was then added, gently mixed and aged for 30 minutes. The reaction was then gently centrifuged, the supernatant removed and the capsules recovered by resuspension in water.

\subsubsection{Synthesis of $\mathrm{Gd}^{3+}$-containing CACs}

An $8.9 \mu \mathrm{M}$ stock solution of PAH was prepared by dissolving PAH (0.0498 g, 0.89 $\mu \mathrm{mol})$ in a $3: 2 \mathrm{v} / \mathrm{v}$ mixture of acetonitrile and water $(10 \mathrm{~mL})$. A stock solution of GdDOTP $^{5-}$ was prepared by diluting a concentrated solution into a $3: 2 \mathrm{v} / \mathrm{v}$ mixture of acetonitrile and water to afford a $0.757 \mathrm{mM}$ stock solution. The $\mathrm{pH}$ of the chelate stock solution was adjusted to 9, by addition of $1 \mathrm{M} \mathrm{NaOH}$ solution. Stock solutions of EDC $(0.174 \mathrm{M})$ and succinic acid $(0.089 \mathrm{M})$ were prepared in pure water. For CACs $R=0.5$, stock solutions of the polymer $(20 \mu \mathrm{L})$ and GdDOTP ${ }^{5-}(120 \mu \mathrm{L})$ were added to a stirred solution of 3:2 v/v MeCN/ $\mathrm{H}_{2} \mathrm{O}(1 \mathrm{~mL})$. Upon addition of the chelate stock solution the reaction rapidly became turbid. The reaction was vortexed at a medium speed for 10 seconds. Aggregates were then allowed to age for 3 minutes before addition of the succinic acid stock solution $(20 \mu \mathrm{L})$. The reaction was vortexed for a further 10 seconds at medium speed and then allowed to age without agitation for 3 minutes. A solution of EDC $(20 \mu \mathrm{L})$ was added and the reaction was aged for 1 hour. Unreacted starting materials were removed by filter centrifugation using $10 \mathrm{kDa}$ MWCO filter centrifuge tube. The CACs retained in the filter centrifuged tube were washed with water and filtered by centrifugation for 30 minutes at $9,000 \mathrm{rpm}$ a total of six times. The CACs 
were then taken up into water $(1 \mathrm{~mL})$ and recovered into a sample vial. All CAC preparations were repeated six times and data are an average of all of these independent capsule preparations.

\subsubsection{Scanning Electron Microscopy}

Scanning electron microscopy (SEM) was performed on a FEI Sirion FEG electron microscope equipped with an energy dispersive X-ray (EDX) detector. A droplet of NACs suspension was placed on the aluminium stub and dried in air, the sample was then sputter coated with gold for 55 seconds. Secondary electron images were taken at $5 \mathrm{kV}$ with a working distance between 5-10mm.

\subsubsection{Transmission Electron Microscopy}

Transmission electron microscopy (TEM) imaging was performed using carbon/copper TEM grids on a JEOL $1200 \mathrm{EX}$ system with an accelerating voltage of 40 to $120 \mathrm{kV}$ and mounted with a Sis Morada 11 Mpixel CCD camera.

\subsubsection{Confocal microscopy}

Confocal images were captured using a TCS-SPE II Leica DM 2500 with 63X magnification and a laser excitation wavelength of $488 \mathrm{~nm}$ for FITC an excitation wavelength of $500 \mathrm{~nm}$ and emission wavelength $555 \mathrm{~nm}$. Scan format was 512 X 512 pixels. Samples were mounted on conventional glass slides and sealed under cover slip to prevent drying 


\subsubsection{Light Scattering}

Dynamic light scattering was performed on a Horiba LB-550 dynamic light scattering instrument. For these measurements freshly syringed filtered samples were dispersed in water and measured at four dilutions to ensure size distributions were independent of concentration effects. Samples were regularly agitated to guard against settling of larger particles.

\subsubsection{Relaxometry}

Water proton $T_{1}$ s were measured on a $0.47 \mathrm{~T}$ Bruker MiniSpec contrast agent analyzer operating at $19.99 \mathrm{MHz}$ using an inversion recovery pulse sequence. NACs were suspended in water $(1 \mathrm{~mL})$ at $\mathrm{Gd}^{3+}$ concentrations ranging from $0.22 \mathrm{mM}$ to $2.37 \mathrm{mM}$. Samples were regularly agitated to guard against settling of larger particles. Relaxivity values were determined by linear regression analysis of the experimentally determined $R_{l}$ values as a function of $\mathrm{Gd}^{3+}$ concentration in Excel.

\subsubsection{ICP-OES Gd ${ }^{3+}$ Concentration Determinations}

Concentration determination of Gd was performed using a Perkin Elmer Optima 2000 inductively coupled plasma optical emission spectrometer (ICP-OES). $\mathrm{Gd}^{3+}$ standards were produced by quantitative serial dilution of a commercial $1000 \mathrm{mgL}^{-1}$ gadolinium in $2 \%$ nitric acid standard (Fluka Analytical) into a $0.1 \%$ nitric acid solution. Samples were prepared by digesting the dried samples with $70 \% \mathrm{HNO}_{3}(100 \mu \mathrm{L})$ and water $(900$ $\mu \mathrm{L})$. The resulting analyte was vortexed thoroughly, and then filtered to remove any particulate using a $0.2 \mu \mathrm{m}$ filter to produce the final sample for ICP-OES analysis. Sample analyte concentrations were calculated to fall in the middle of the constructed 
ICP-OES Gd ${ }^{3+}$ calibration curve to ensure accurate Gd spectral readings. Readings were taken in triplicate and averaged. The highest percent relative standard deviation allowed between these replicates was $1 \%$, to ensure precise $\mathrm{Gd}^{3+}$ spectral readings for the samples analyzed in this work. The concentration of $\mathrm{Gd}^{3+}$ was determined for each sample and then multiplied by the dilution factor.

\subsubsection{Results and Discussions}

The first step of the CAC preparation is identical to that of NACs. An initial preparation of CACs was performed using pyrophosphate as anion and PAH as the cationic polymer to form an aggregate in pure water at a mild $\mathrm{pH}$ by Sally Plush. L-Glutamic acid was then used to create a coating of the surface of the aggregate, interacting with the free amino groups that are available on the aggregate surface. The coated aggregates were then crosslinked by addition of a peptide coupling reagent 1- ethyl-3-(3-dimethylamino propyl) carbodiimide (EDC). Capsules were purified by centrifugation and resuspendend in water. TEM images show the presence of the capsules as shown in Figure 3.17. This new approach to encapsulation was investigated in terms of its ability to encapsulate $\mathrm{Gd}^{3+}$ chelates. The same methodology was employed to encapsulate GdDOTP ${ }^{5-}$, which was mixed with $\mathrm{PAH}$ (the cationic polymer) in $3: 2 \mathrm{MeCN} / \mathrm{H}_{2} \mathrm{O}$ at $\mathrm{pH} 9 . R$ ratios from 0.3 to 1.0 where investigated and in each case a turbid solution rapidly formed a sign of the formation of an aggregate. Succinic acid was used as the coating dicarboxylic acid on the surface of the aggregate. EDC was used, as before, as the cross linker. After purification by centrifugation through a $10 \mathrm{kD}$ MWCO centrifuge filter $\mathrm{Gd}^{3+}$-containing CACs were obtained. Formation of CACs is shown on the SEM images Figure 3.17. 


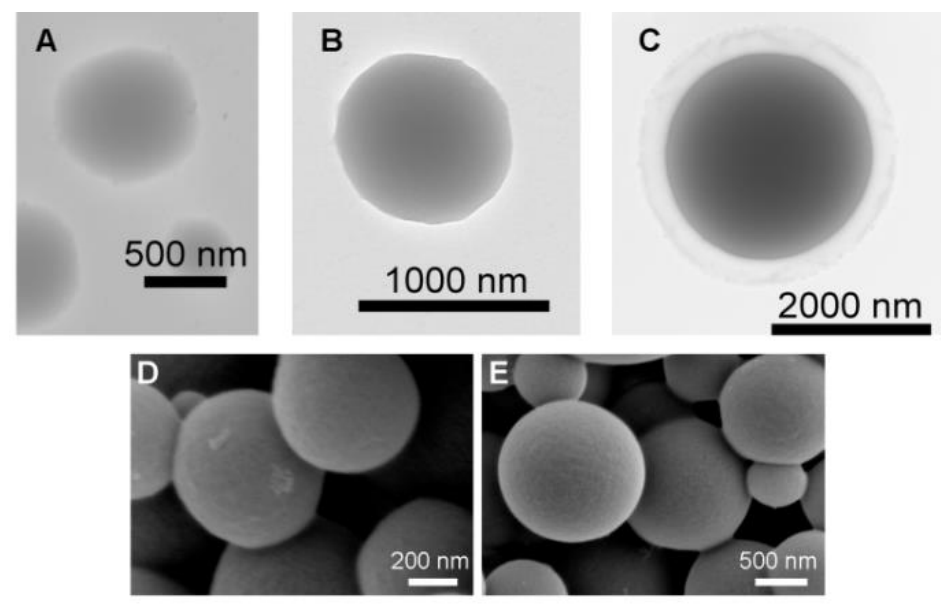

Figure 3.17: Electron micrographs of different stages of CAC preparation. A) TEM image of a single pyrophosphate/PAH aggregate $(R=5)$ showing the loose morphology of the aggregate; $B)$ TEM image of a pyrophosphate/PAH aggregate with a glutamate coating showing the tighter surface morphology of the coated aggregate; C) TEM image of a pyrophosphate/PAH CAC after treatment with EDC showing the crosslinked capsule shell; and SEM images of GdDOTP ${ }^{5} / P A H C A C s$ after treatment with succinic acid and EDC D) $R=0.5$ and $E$ ) $R=1$.

The hydrodynamic diameter of the CACs was determined by dynamic light scattering.

The size of CACs was found to be dependent on the charge ratio $R$ in a similar way to NACs: the smaller the $R$ value the smaller the CACs observed and vice versa. 


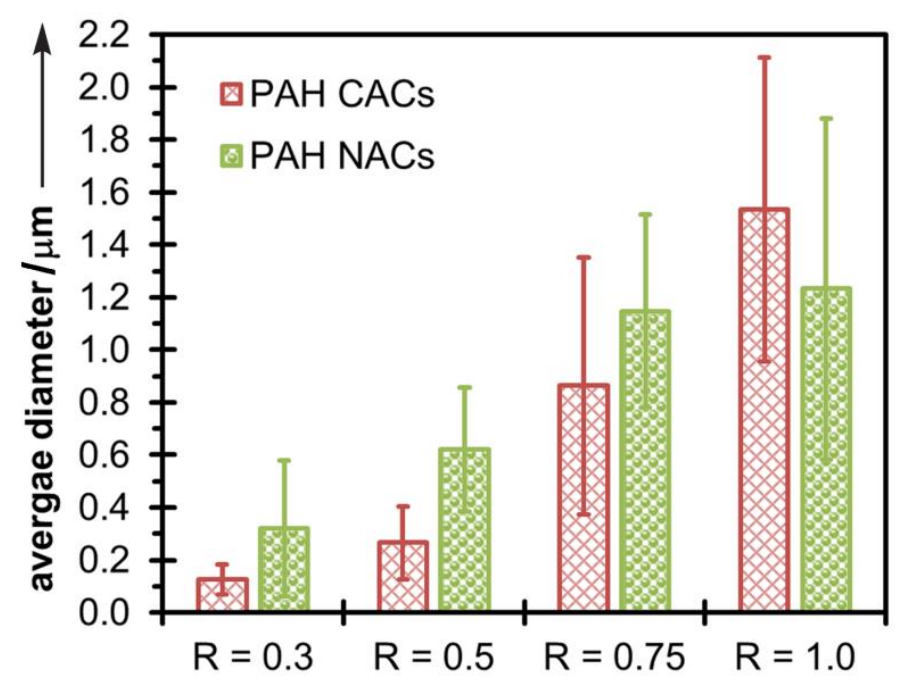

Figure 3.18: A comparison of CACs (red) and NACs (green) prepared using PAH and GdDOTP5- average diameter. Each value is the mean of six nano capsule preparations

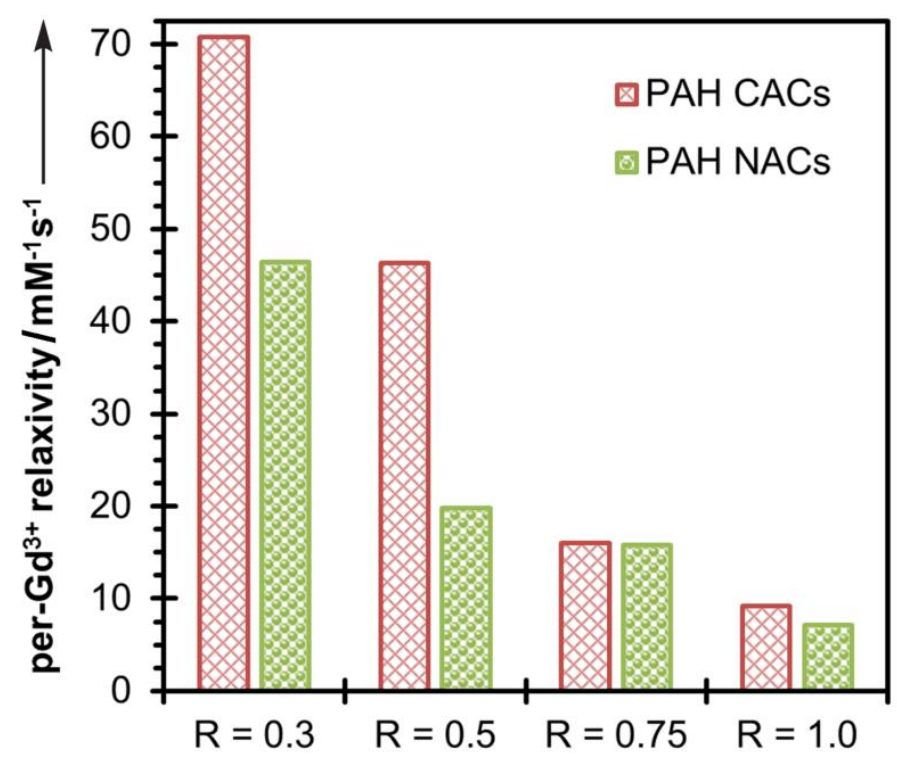

Figure 3.19: A comparison of CACs (red) and NACs (green) prepared using PAH and GdDOTP ${ }^{5-}$ contrasting the per $\mathrm{Gd}^{3+}$ longitudinal relaxivity $(20 \mathrm{MHz}$ and $298 \mathrm{~K}$ ). Each value is the mean of six nano-capsule preparations

The smallest capsules were attained $(R=0.3)$ from this method were almost half the size of the NACs produced using the same value of $R$ and chelate $\left(\mathrm{GdDOTP}^{5-}\right)$ as shown in 
Figure 3.18. It is notable that the trend for the size of NACs and CACs observed as $R$ changes is not identical, although CACs are smaller than NACs when prepared with low $R$ values they are slightly larger than NACs when higher $R$ values are employed.

The smaller size of $R=0.3$ CACs versus NACs has a substantial impact on relaxivity. The measured relaxivity, $70.2 \mathrm{mM}^{-1} \mathrm{~s}^{-1}(20 \mathrm{MHz}, 25 \mathrm{C})$ represents an almost 2-fold enhancement in relaxivity over the NACs (Figure 3.19) and a 15-fold enhancement over GdDOPT $^{5-}$ alone. In all cases the per $\mathrm{Gd}^{3+}$ relaxivity correlated with the size of the capsule formed; as the charge ratio increases there was a decrease in relaxivity and an increase in size of capsules (Figure 3.19). The larger the capsule size the longer the distance the proton close to the chelate has to diffuse to exchange with bulk water. The higher relaxivity values found for CACs relative to NACs, even in the case of $R=1.0$ (in which the CAC is larger than the NAC) is an indication that the cross-linked coating of CACs is and least as, and probably more, permeable to water than the $\mathrm{SiO}_{2} \mathrm{NP}$ shell of NACs. Interaction of the GdDOTP ${ }^{5-}$ with the cationic polymer PAH in the aggregate is anticipated to limit the tumbling of the chelates in solution; this is the cause of the large increase in overall relaxivity. Smaller capsules have higher relaxivity compared to larger capsules because proton (or whole water) exchange between the chelate and bulk water is faster (diffusion being proportional to the reciprocal of the diameter). GdDOTP ${ }^{5-}$ aggregated into larger capsules do not have such high relaxivity values which shows that encapsulation does play a role in limiting diffusion of water proton even though a robust shell for encapsulating is needed if these nano assembled capsules are to be used in vivo. 
Table 3: The average hydrodynamic diameter $(\mu \mathrm{m})$ determined by dynamic light scattering of GdDOTP ${ }^{5-}$ containing nano capsules (CACs and NACs) prepared with PAH in 3:2 MeCN/H2O as a function of the $R$ value used in their preparation. Data are presented with the $1^{\text {st }}$ population standard deviation

\begin{tabular}{cccc}
\hline & CACs & NACs & \% Gd incorp \\
\hline $\mathrm{R}=0.3$ & $0.127 \pm 0.057$ & $0.32 \pm 0.26$ & 99.2 \\
$\mathrm{R}=0.5$ & $0.265 \pm 0.138$ & $0.62 \pm 0.235$ & 98.7 \\
$\mathrm{R}=0.75$ & $0.863 \pm 0.488$ & $1.147 \pm 0.368$ & 98.6 \\
$\mathrm{R}=1.0$ & $1.534 \pm 0.58$ & $1.232 \pm 0.65$ & 99.5 \\
\hline
\end{tabular}

The percentage encapsulation of GdDOTP $^{5-}$ into CACs was found to be $\geq 98 \%$, comparable to the rate of incorporation into NACs. The high rate of incooperation of GdDOTP $^{5-}$ into these capsules is correlated to the efficient interaction of GdDOTP ${ }^{5-}$ with the polymer PAH which in turn afford high payloads of the chelate per capsule.

\subsubsection{2: Probing the stability of cross linked assembled capsules (CACs)}

A synthesis method has been developed to synthesize nano-scale capsules loaded with high payloads of high relaxivity MRI contrast agent. The cross-linked encapsulating shell of these capsules affords the promise of high stability and robustness, rendering the capsules more appropriate for in vivo applications. To test this resilience of CACs to different $\mathrm{pH}$ regimes and metal ion concentration was examined. Suspensions of CACs were incubated at $\mathrm{pH} 3,5,7,9$ and 11 and in the presence of $5 \mathrm{mM} \mathrm{CaCl}_{2}$ and $5 \mathrm{mM}$ $\mathrm{MgCl}_{2}$ and $200 \mathrm{mM} \mathrm{NaCl}$. These values exceed the range of $\mathrm{pH}$ and metal ion concentration found in physiological fluids. In addition to the long term stability of the 
CACs, the effect of metal ions on relaxivity was also of interest, since both $\mathrm{Ca}^{2+}$ and $\mathrm{Mg}^{2+}$ are known to bind to GdDOTP ${ }^{5-}$, quenching relaxivity. The CACs were analyzed in terms of their size (DLS), relaxivity ( $T_{l}$ relaxometry) and morphology (SEM).

\subsubsection{3: The effect of $\mathrm{pH}$ on CAC Stability}

The structure of cross linked assembled capsules is held together by the peptidic bonds of the encapsulating shell. It is reasonable to expect that these bonds and the electrostatic interactions in the CAC interior may be disrupted by changes in $\mathrm{pH}$. To examine any response to changes in $\mathrm{pH}$ on the structure of $\mathrm{CACs}$, they were dispersed in solutions of $\mathrm{pH} 3,5,7,9$, and 11 and incubated for 4 weeks. The solutions were prepared by combining deionized water, $\mathrm{HCl}(12 \mathrm{M})$ or $\mathrm{NaOH}(5 \mathrm{M})$ to achieve the desired $\mathrm{pH} .1 \mathrm{~mL}$ of a CAC suspension was placed in a dialysis membrane which was placed in a solution at the $\mathrm{pH}$ to be studied. Capsules were removed from the dialysis membrane after each week for characterization. Before making relaxation measurements capsules were washed twice with DI water to make sure that no influence on the relaxivity values is a contribution from the salts. No apparent change in the size of the capsules was observed over four weeks at any $\mathrm{pH}$ studied (Figure 3.21). Furthermore, SEM images show no notable change in capsules morphology over the same period. 

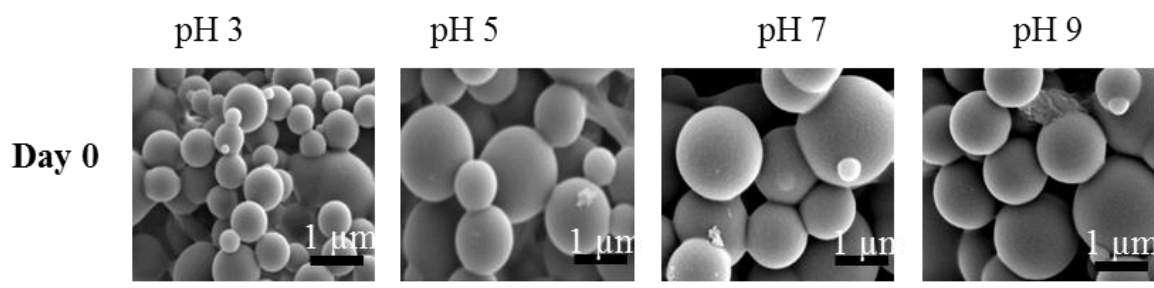

$\mathrm{pH} 11$

Day 28
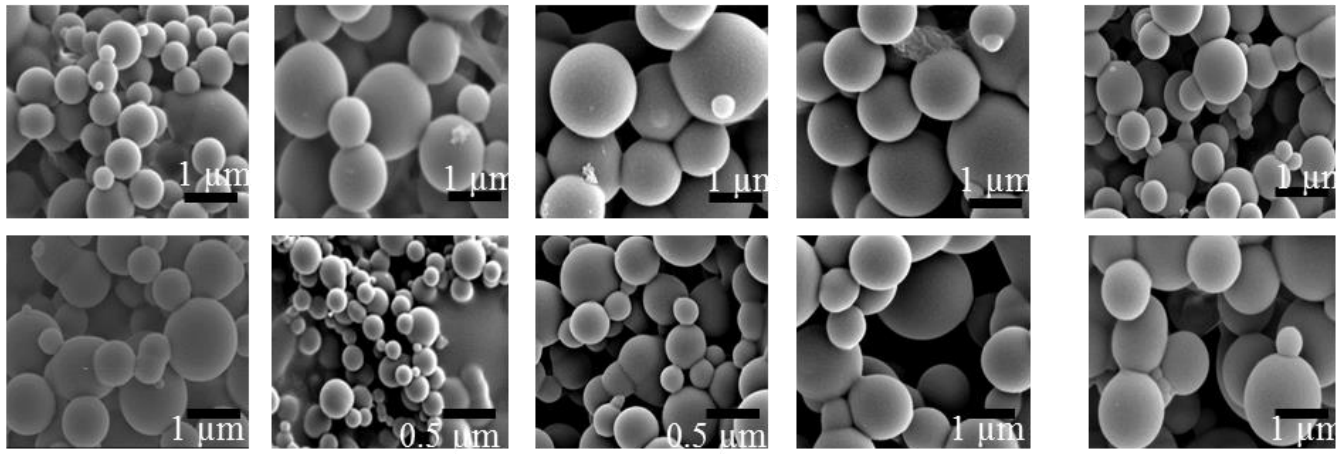

Figure3.20: SEM images of CACs exposed to $\mathrm{pH} 3-11$ from day 0 to day 28 for $R=0.3$

The per- $\mathrm{Gd}^{3+}$ relaxivity for CACs incubated between $\mathrm{pH} 3$ and $\mathrm{pH} 9$ remains essentially constant over the course of 4 weeks. This suggests that in addition to the capsules retaining their shape and size, there is no appreciable release of the chelate or change in the protons (or water) transfer rate in the $\mathrm{pH}$ range 3-9. In contrast, at $\mathrm{pH} 11$, a continuous superlinear drop in relaxivity was observed for $\mathrm{pH} 11$ over the course of the experiment. Given the capsules do not seem to change size or shape significantly at this $\mathrm{pH}$ the most likely explanation for this drop in relaxivity is base catalysed hydrolysis of the $\mathrm{Gd}^{3+}$ ion from DOTP. This would produce the insoluble $\mathrm{Gd}(\mathrm{OH})_{3}\left(\mathrm{H}_{2} \mathrm{O}\right)_{6}$ which would account for the drop in relaxivity. 


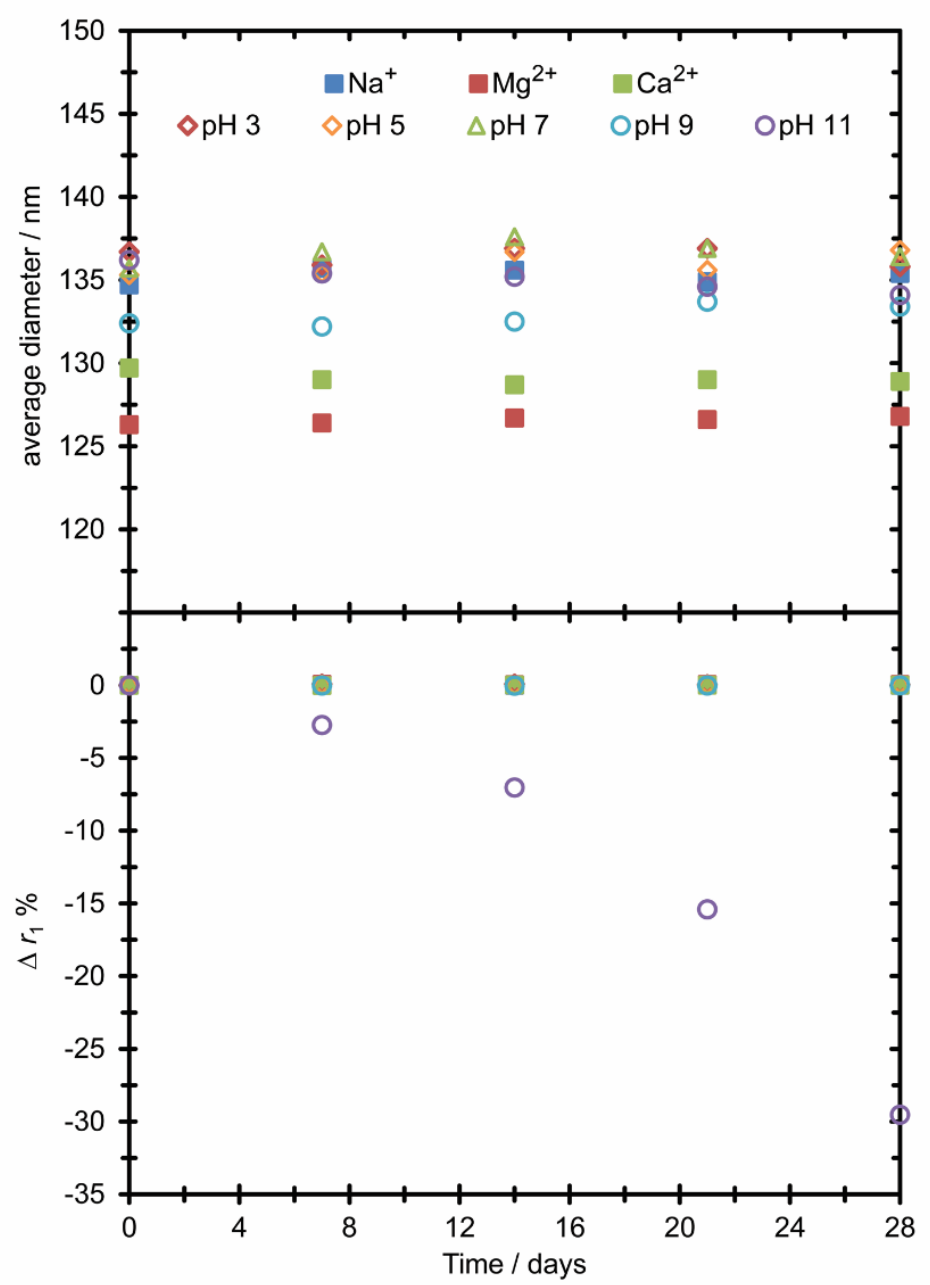

Figure 3.21: A comparison of CAC\% change in $G d 3+$ relaxivity and average size of capsules over 28 days at different $\mathrm{pH}$ values $\mathrm{pH}$ 3(red diamonds), $\mathrm{pH} 5$ (orange diamonds), $p H 7$ (green triangles), pH 9(blue circles), pH 11(purple circles) and salts $\mathrm{NaCl}$ (blue circles), $\mathrm{CaCl}_{2}$ (green squares) and $\mathrm{MgCl}_{2}$ (red squares

\subsubsection{4: The effect of metal ions on CAC stability.}

In biological fluids several metal ions are abundant that may modulate the strength of electrostatic interactions. To study whether some of the most abundant metal ions affect the function of CACs as MRI contrast agents, CACs were suspended in dialysis membranes in solutions of $5 \mathrm{mM} \mathrm{CaCl}_{2}, 5 \mathrm{mM} \mathrm{MgCl} 2$ and $200 \mathrm{mM} \mathrm{NaCl} . \mathrm{Ca}^{2+}$ 
accomplishes most of its biological process in the presence of $\mathrm{Mg}^{2+}$ so it was important to study both these ions on their effect on the CACs. The experimental design was the same as that used for the $\mathrm{pH}$. CACs were suspended in dialysis membranes in solutions of the metal ion of interest. The capsules were sampled each week during the 4 weeks of incubation in solutions of metal ions. Capsule size was measured by DLS and the relaxation rate constants were measured at day 0 and each 7 days thereafter. SEM images were taken (Figure 3.22) at the beginning and end of the incubation period to assess changes to the morphology of the capsules. Size of the capsules was not affected by incubation in the presence of the endogenous metal ions $\mathrm{Ca}^{2+}, \mathrm{Mg}^{2+}$, and $\mathrm{Na}^{+}$. Furthermore, no change was observed in the relaxivity value of these capsules (Figure 3.19). This is of particular relevance in the case of $\mathrm{Mg}^{2+}$ and $\mathrm{Ca}^{2+}$, both of which are known to bind to GdDOTP ${ }^{5-}$ and quenching relaxivity. This suggests that the polymer hydrogen bonds to the phosphonate groups in such a way as to block the metal ion binding, which quenches relaxivity. These salts also seem to have no effect on the electrostatics of the capsules, no disassembling of these capsules was observed based on the size analysis and the morphology studies using the SEM (Figure 3.22). 

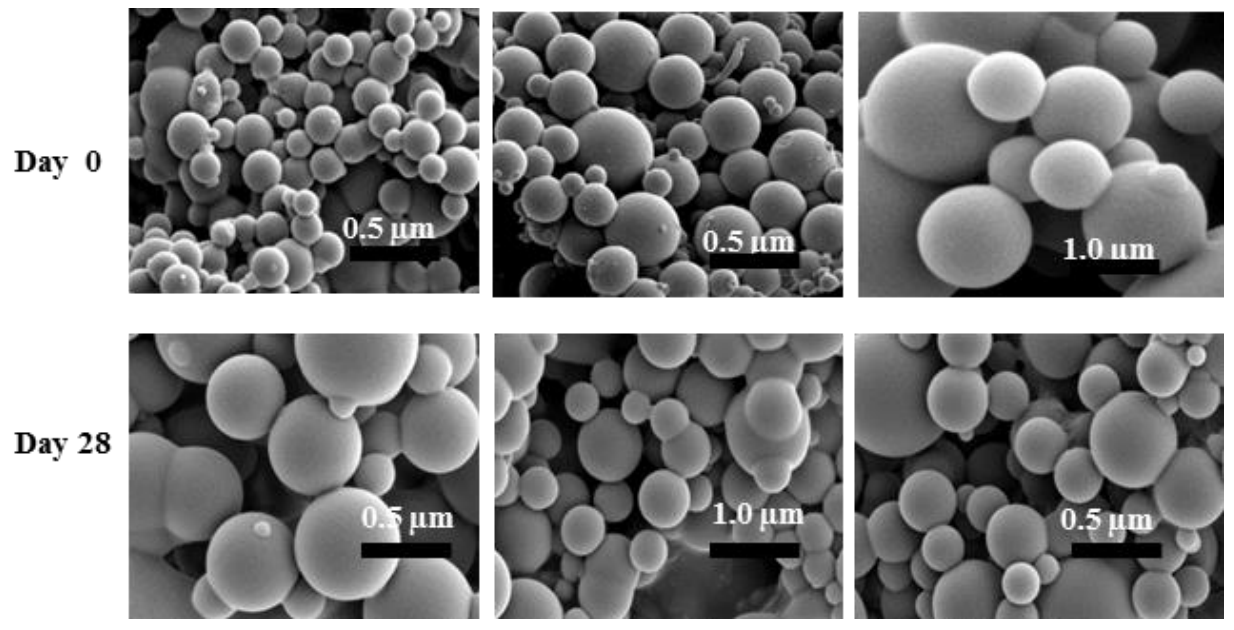

Figure 3.22: SEM images of CACs suspended in $5 \mathrm{mM} \mathrm{CaCl2,} \mathrm{MgCl}$ and $\mathrm{NaCl}$ for day 0 and day 28respectively

MRI offers high spatial resolution, but in order to be used for molecular imaging applications it is necessary to deliver a high payload of $\mathrm{Gd}^{3+}$ to a specific pathology. CACs represent a solution to delivering high payloads of the $\mathrm{Gd}^{3+}$ chelates to the target site. Moderately sized $(\leq 30 \mathrm{kDa})$ targeted contrast agents are often preferred because their small size enables them to diffuse freely to small areas and their simple clearance, but this type of targeted imaging tend to suffer low sensitivity with small $\mathrm{Gd}^{3+}$ based contrast agents. ${ }^{119,} 120$ Contrast agents that possess high relaxivity and the capability to deposit high payloads of contrast agents are more desirable from a detectability point of view. This may limit the applications for which they can be used by their distribution characteristics but their use will not have the same detection limitations. The development of such an agent may be envisioned through the attachment of a targeting moiety on the surface of the CAC. A lot of work has been performed by conjugating targets $^{112}$ and many other techniques which allows attachment of targeting moieties on the surface or link them to contrast agents. Selective coupling of primary amino groups 
to isothiocyanate is one of the methodologies which have been used under mild conditions. To investigate whether it is possible to undertake such a coupling reaction on the surface of $\mathrm{Gd}^{3+}$ chelates containing CACs, a sample of the prepared $\mathrm{R}=0.5$ CACs where treated with FITC, an isothiocyanate was dissolved in DSMO at $1 \mathrm{mg} / \mathrm{mL}$ and was prepared fresh for each labelling and for every $1 \mathrm{~mL}$ of CACs, $50 \mu \mathrm{L}$ of FITC solution was added. CACs were suspended in $0.1 \mathrm{M}$ sodium carbonate buffer at $\mathrm{pH} 9$ and the buffer was prepared fresh, to make sure that $\mathrm{pH}$ of the buffer does not change upon storage. Thorough washing was performed on the CACs to remove unreacted dye and CACs were imaged using a confocal microscope (Figure 3.23). The intense green emission from the capsules indicates that FITC was able to access and react with primary amino groups on, or close to, the surface of capsules. This shows that it should be possible to conjugate a targeting moiety on the surface of the CAC producing targeted high payload contrast agents with enhanced relaxivity. Increasing the payload by synthesizing the $\mathrm{CAC}$ has shown to give high per- $\mathrm{Gd}^{3+}$ relaxivity values and incorporating many high relaxivity contrast agents in each capsule affords exceptionally high per-capsule relaxivities. This means that each capsule will have very significant effects on water proton $T_{1}$ s reducing the detection limit of any agent targeted to a specific pathology of interest in molecular imaging applications. We have also investigated the availability of primary amino functions on the surface of CACs with FITC to quantify the availability of these functional groups. 


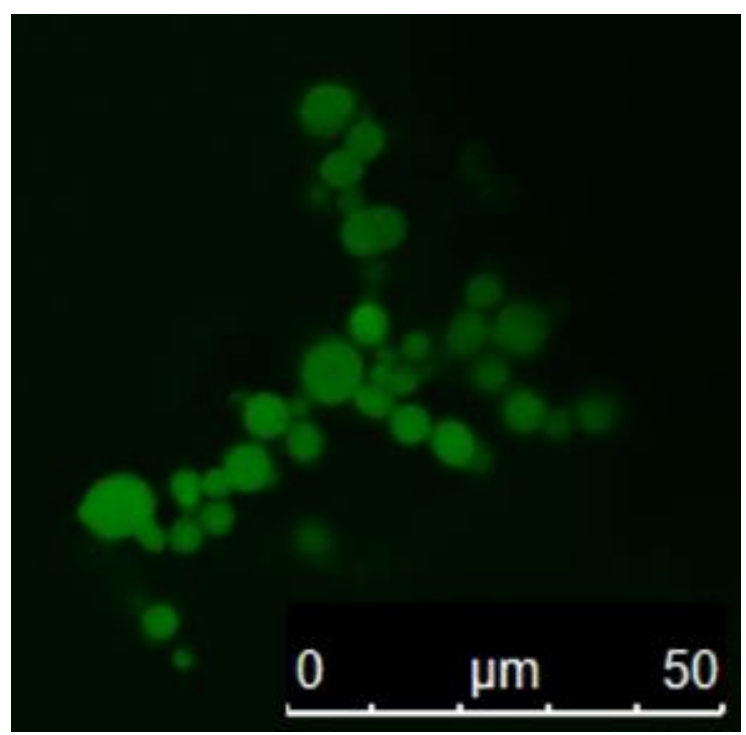

Figure 3.23: Bright field optical field microscopy image of $R=0.5$ CACs prepared with $G_{D O T P}{ }^{-}$and treated with FITC after. 63xmagnification; excitation laser line $488 \mathrm{~nm}$; excitation wavelength $500 \mathrm{~nm}$; emission wavelength $555 \mathrm{~nm}$; scan format $512 \times 512$ pixels

\subsubsection{Conclusions}

A new method for robust encapsulating nano-assembled capsules has been developed. This method is tolerant of charge ratios $(R)$ from 0.3 to 1.0 , beyond a charge ratio of 1 capsule were difficult to synthesize. There was a stronger but similar relationship between the value of $R$ and the size of capsules than for NACs. The smallest capsules where obtained by using this alternative method of coating combined with a low $R$ value. These very small capsules $(127 \mathrm{~nm})$ afforded the highest relaxivity values to date, almost twice as high as those of capsules coated with $\mathrm{SiO}_{2}$ nanoparticles. The interaction of GdDOTP $^{5-}$ with the cationic polymer limits the freedom of motion of the chelate and brings exchangeable protons into close contact with the chelate through the "secondsphere". ${ }^{121,122}$ This is the origin of the enhanced relaxivity. The alternative coating method allows movement of water molecules across the coating and does not have a 
limiting effect on relaxivity. The surface of CACs possess free amines which have been shown to be reactive to external labelling approaches, such as FITC a fluorescent agent as demonstrated by confocal microscopy. The availability of reactive free amines on the surface of CACs is an added advantage of these capsules because they provide a method for attaching targeting moieties to the surface of these capsules. The stability of CACs were also explored in this chapter for different metal ions $\left(200 \mathrm{mM} \mathrm{Na}{ }^{+}, 5 \mathrm{mM} \mathrm{Ca}^{2+}\right.$ and $5 \mathrm{mM} \mathrm{Mg}^{2+}$ ) and $\mathrm{pH}$ values (3-11). CACs were found to be robust in the presence of all of the metal ions studied for the duration of the four week experiment. The relaxivity remained constant for four weeks and no detectable change in the size or morphology of the CACs was observed. The effect of incubating CACs in solutions from $\mathrm{pH}$ 3-9 was found to be similar. However, at $\mathrm{pH} 11$ incubation has a substantial effect on relaxivity (presumably the result of metal hydrolysis) but the size and morphology of the CACs seem to be unaffected. We can conclude that CACs are stable against many of the likely sources of interference from the constituents of extracellular fluids and represent good candidates for the development of high relaxivity, high payload molecular imaging agents. 
Chapter 4. Synthesis of a reverse NAC as a paraCEST agent

\subsection{Introduction}

MRI is a clinical diagnostic method widely used in the medical field for anatomical imaging, owing to its spatial and anatomical resolution. $\mathrm{Gd}^{3+}$ complexes are widely used as MRI contrast agents in clinical diagnostic medicine. These MRI agents lack tissue specificity and they do not respond to their chemical environment, therefore work has been performed where MRI contrast can be altered based on chemical exchange saturation transfer (CEST). Paramagnetic metal complexes that can shift the frequency of an exchanging site in a chelate far from the bulk water signal may be able to function as a CEST agent. Lanthanides ions such as $\mathrm{Eu}^{3+}, \mathrm{Ho}^{3+}, \mathrm{Dy}^{3+}, \mathrm{Yb}^{3+}$ and $\mathrm{Tm}^{3+}$ have near ideal properties as paramagnetic CEST agents. Complexes formed from these ions and neutral ligands such as tetramides derivates of DOTA has the slower water exchange rates required of CEST agents. Unlike $\mathrm{Gd}^{3+}$, these lanthanide metals have low relaxivity but when operating as paraCEST they maintain an ability to effect changes on image contrast $^{123}$. One of the major advantages of using paraCEST agents is the ability to turn them on and off by changing the pulse sequence parameters. It is only when a presaturation pulse is situated at the resonance frequency of the bound water molecule or exchangeable amides protons will the image exhibit CEST contrast. A pre-saturation pulse at any other frequency will not affect the image. And because these paraCEST agents have very low relaxivities they do not substantially alter the image through

changes in $T_{1}$ or $T_{2}$. This feature is unlike $\mathrm{Gd}^{3+}$ chelates which are always on and affect the image until the $\mathrm{Gd}^{3+}$ chelate has been cleared from the body. This limits the timing of 
the acquisition of pre- and post-contrast images. In molecular imaging applications it is envisioned that the post-contrast image will be acquired after administration of the contrast agent and after the agent has cleared from healthy tissue. This will clearly be a long time after the acquisition of the pre-contrast image, creating problems for the coregistration of the two images. A contrast agent, such as paraCEST agent, that can be switched on and off will offer a significant advantage because the pre- and post-contrast images can be collected one after the other faciltating better co-registration of the images.

One of the major limitations of CEST is the inherent sensitivity of paraCEST agents. However, entrapping paraCEST agents in nano-scale capsules could deliver a large payload of a paraCEST agent and improve the limit of detection for these agents. This opened new avenues for the discovery of new classes of responsive agents like MR gene reporters to small molecules that respond to biological events. Since the first demonstration of paraCEST contrast agents by Zhang and his co workers in $2001^{82}$, they have been explored for a number of biomedical applications for different targets $\mathrm{pH}^{72,124}$, temperature $^{125,}{ }^{126}$, enzymes ${ }^{127,}{ }^{128}$, metabolites ${ }^{129-131}$ and metal ions. ${ }^{132,}{ }^{133}$ Use of paraCEST contrast agents have shown promise in in vivo system application. The biggest challenge of paraCEST contrast agents method in in vivo is loss of sensitivity and poor temporal resolution for detection, due to magnetization transfer from endogenous macromolecules following application of radio frequency pulse that saturates bulk water spins lowering the contrast efficiency of ParaCEST agents. Currently available ParaCEST agents are inherently insensitive as imaging agents, therefore they require agents in the millimolar range. A number of approaches for increasing the proton or 
water exchange rates and the number of exchangeable groups per agent, and the latter approach seems to be more promising $87,134,135$, therefore incooperation of contrast agents into nano assembled capsules is another way to increase contrast agent localization, it is a more effective way to increase contrast agent molecules to a region with highly loaded capsules with the contrast agent. There are a number of nanoparticle strategies that have been investigated to enhance the sensitivity of CEST contrast agents. Entrapment of CEST contrast agents involves binding the CEST agent to the nanoparticle structure physically or trapping the contrast agent and chemically attaching a CEST agent both diaCEST and ParaCEST agents have been demonstrated to be trapped using polymers ${ }^{136-}$ ${ }^{138}$, liposomes ${ }^{111,139}$ and dendrimers ${ }^{87,140}$ and where able to generate a CEST effect. In this chapter we investigated one system where we synthesized a reverse NAC by incorperating a traditional ParaCEST DyDOTAM ${ }^{3+}$.

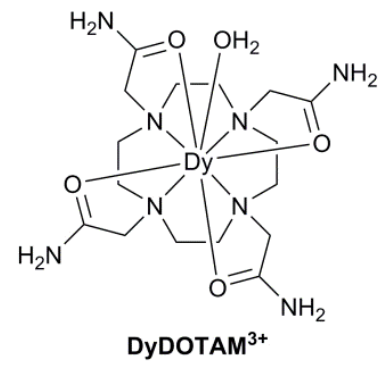

Scheme 6: The structure of the chelate used in the synthesis of the reverse NACS

\section{2: Experimental}

\subsection{1: General remarks}

A solution of polyacrylic acid (100,00 MW acrylic units per molecule, $35 w t \%$ in water) and poly allyamine hydrochloride (56, $000 \mathrm{MW} \sim 596$ allylamine units per molecule) were purchase at sigma Aldrich. Ethylene diamine (EDA) was purchased from J.T 
Baker. A suspension of $\mathrm{SiO}_{2} \mathrm{NP}$ (Snowtex-O type, 20.3 wt \% $\mathrm{SiO}_{2} \mathrm{NP}$, and $\mathrm{pH} 3.5$ was purchased from Nissan chemicals and the $\mathrm{pH}$ adjusted to 5.5 using $150 \mathrm{mM} \mathrm{NaOH}$ prior to use. Deionized water (18.2 M $\Omega$, Barnstead Nanopure Diamond System) was used in all reactions

\subsection{2: Reverse NAC synthesis}

An aqueous solution of PAA $(215 \mu \mathrm{L}, 5 \mathrm{mg} / \mathrm{mL})$ was mixed with an aqueous DyDOTAM $^{3+}$ solution $(535 \mu \mathrm{L}, 40 \mathrm{mM})$ in a $1.5 \mathrm{~mL}$ eppendorf centrifuge tube and vortex mixed for 10 seconds at low speed. A charge ratio $R=0.5$ (equation 2.1) was studied in this work, the EDA concentration was $0.11 \mathrm{M}$. A turbid mixture immediately resulted, indicating the formation of PAA-DyDOTAM aggregates the reaction mixture was vortexed at a very low speed for 5 seconds and aged for 10 seconds, and an aqueous solution of PAH $(20 \mu \mathrm{L}, 89 \mu \mathrm{M})$ was added to the turbid solution of PAA-DyDOTAM aggregates and vortexed at a low speed for 10 seconds and aged for 15 seconds. EDA was added $(215 \mu L)$ to the turbid solution and there was an increase in turbidity. This suspension was aged for 10 seconds, before an aqueous $\mathrm{SiO}_{2} \mathrm{NPs}$ suspension $(535 \mu \mathrm{L}$; concentration of $2.0 w t \%$ ) was added. The resultant mixture was stirred vigorously at a medium speed for 10 seconds and aged for 2 hrs. Unreacted starting materials were removed by filter centrifugation using $10 \mathrm{kDa}$ MWCO filter centrifuge tube.

\section{3: Results and Discussion}

It has been successfully shown in our earlier work that NACs are a versatile platform for the development of high payload contrast agents. However, assembly had previously been limited to a cationic polymer, multivalent anionic contrast agents, and negatively 
charged NPs. We wondered whether NAC synthesis could be made in a reverse assembly methodology, comprising an anionic polymer, multivalent cationic contrast agents, and negatively charged NPs. The motivation for this reversal is the possibility to incorporate a positively charged paraCEST contrast agent into the capsule. For this reason $\mathrm{Gd}^{3+}$ is replaced by $\mathrm{Dy}^{3+}$, because $\mathrm{Dy}^{3+}$ has near ideal properties as a paramagnetic CEST agent when chelated with a neutral ligand such as DOTAM. Dy ${ }^{3+}$ significantly shifts the amide protons of the ligand which are in slow exchange with water; however, once inside a capsule it is not clear that this chelate will continue to function as a CEST agent since the rate at which protons exchange may change due to hydrogen bonding interactions. However, if exchange remains in the slow regime then the concept of reverse assembly may facilitate a method by which high loadings of positively charged chelates can be achieved. In addition to high payloads of CEST agent, one other approach to increasing the sensitivity of CEST is to increase the number of exchangeable protons per agent. For this reason DyDOTAM ${ }^{3+}$ was chosen as the CEST agent. In addition to 2 exchanging water protons shifted to $-760 \mathrm{ppm}$, this chelate has 8 amide protons that can exchange slowly with water shifted to $+90 \mathrm{ppm}$. To achieve the reverse NAC synthesis DyDOTAM ${ }^{3+}$ was mixed with the anionic polymer polyacrylic acid (PAA) in an aqueous solution and PAA at $\mathrm{pH}$ of 7. Upon mixing the solution rapidly went turbid, a strong indication of aggregate formation. Encapsulating this aggregate proved more challenging because the aggregate surface is predominantly negatively charged as are $\mathrm{SiO}_{2} \mathrm{NPs}$. For this reason initial attempts at encapsulation were performed using EDC and polyamines in an attempt to cross-link the capsule shell (c.f. Chapter 3). However, this approach, while theoretically viable, currently remains 
unsuccessful. An alternative strategy was therefore pursued in which the aggregate surface was charge modified prior to addition of $\mathrm{SiO}_{2} \mathrm{NPs}$. A solution of the cationic PAH was added to the aggregates after 10 seconds of aging followed by ethylene diamine and aggregates aged for 10 seconds before $\mathrm{SiO}_{2} \mathrm{NPs}$ were added to encapsulate the, now positively charged, aggregate. Spherical capsules were formed which appear similar by SEM to those formed using PAH and anionic chelates (Figure 4.24). The size of capsules was measured using dynamic light scattering and was found to be $650 \pm 250 \mathrm{~nm}$. These reverse NACs are much larger than the NACs prepared using PAH and anionic chelates at the same charge ratio NACs. For example, NACs prepared with the trianionic chelate GdTTHA $^{3-}$ had an average size of $180 \mathrm{~nm}$, less than a third of the size of the $650 \mathrm{~nm}$ determined for the reverse capsules. It is speculated that this difference arises from differences in the strength of the interaction of the anion and cation in the aggregate, the additional polymer layer than needed to be added and a difference in the interaction of the $\mathrm{SiO}_{2} \mathrm{NPs}$ with this additional polymer layer, versus the $\mathrm{PAH}$ of the aggregates in the NACs.
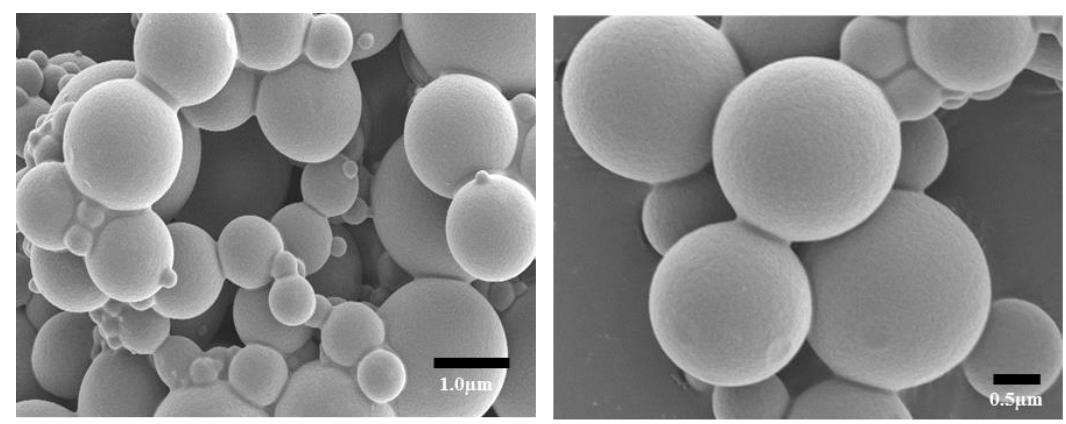

Figure 4.24: SEM images for reverse NACs for DyDOTAM/PAA for $R=0.5$ 


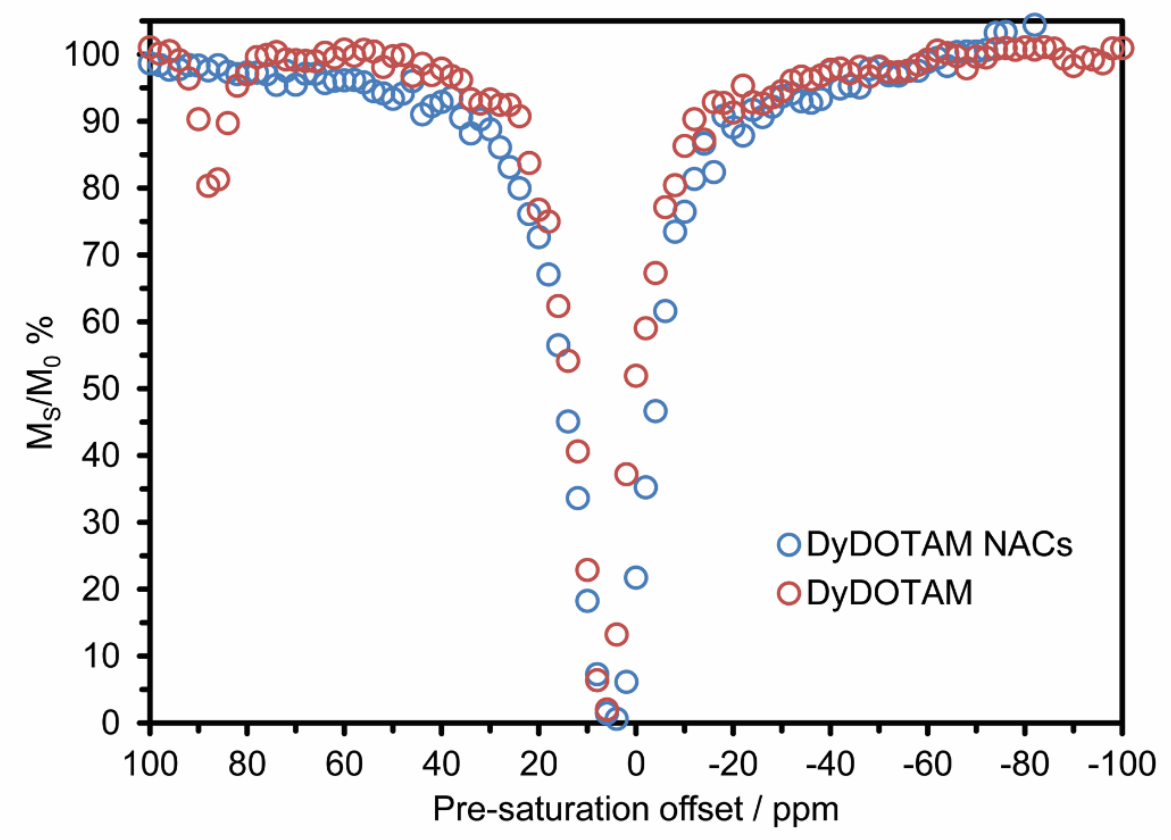

Figure 4.25: CEST spectra of $40 \mathrm{mM}$ DyDOTAM chelate solution (red circles) and 40 mM DyDOTAM NACs (blue circles)

The CEST spectrum of DyDOTAM ${ }^{3+}$ is shown in Figure 4.25. A CEST peak arising from DyDOTAM ${ }^{3+}$ is clearly visible at +90 ppm from the bulk water, which is known to arise from the 8 amide protons. However, the CEST spectrum of DyDOTAM ${ }^{3+}$ containing reverse NACs shows no CEST peak at all in this part of the spectrum. This disappointing result may have several causes: T1/T2, concetration, acceleration of exchange beyond the slow exchange regime, inihibition of exchange by aggregate formation. In these experiments CEST from the bound water molecule at $-760 \mathrm{ppm}$ was not investigated. However, going forward this is an important factor to investigate since one may envision amide protons interacting directly with the PAA of the aggregate. In contrast, the bound water may have no direct interaction at all and its CEST properties may be largely unaffected by encapsulation it was difficult to observe a CEST signal for 
encapsulated DyDOTAM ${ }^{3+}$ NACs at this chemical shift. We speculated that encapsulation of this chelate reduces rotational correlation time and lengthens $R_{I}$ and $R_{2}$ this has a consequence that direct saturation of the water protons surpasses the CEST effect, hence we did not observe a CEST signal from these reverse NACs.

\section{5: Conclusions}

A novel approach was developed to synthesize reverse NACs containing paraCEST agents and CEST data was acquired to assess the effect of encapsulation. No observable CEST was observed from the amide protons of DyDOTAM ${ }^{3+}$ upon encapsulation of the chelates. We speculate that as we encapsulate the chelate there is an increase in exchange rate and it increased a maximum and $\Delta \omega$ begins to broaden and decrease and the exchange peak shifts towards the bulk water frequency $(0 \mathrm{ppm})$. This causes the CEST signal to disappear completely when the exchange is too fast hence no observable difference between the bound and bulk water protons can be observed. There is still a lot to be investigated on the concept of reverse paraCEST agents. 


\section{References}

1. M. Yazdani, C. T. Lau, J. K. Lempel, R. Yadav, A. H. El-Sherief, J. T. Azok and R. D. Renapurkar, RadioGraphics, 2015, 35, 1245-1262.

2. D. Kunio, Physics in Medicine and Biology, 2006, 51, R5.

3. D. J. Brenner and C. D. Elliston, Radiology, 2004, 232, 735-738.

4. S. M. Bierig and A. Jones, Journal of Diagnostic Medical Sonography, 2009, 25, $138-144$.

5. G.-L. Davies, I. Kramberger and J. J. Davis, Chemical communications (Cambridge, England), 2013, 49, 9704-9721.

6. K. T. Spencer, A. S. Anderson, A. Bhargava, A. C. Bales, M. Sorrentino, K. Furlong and R. M. Lang, J Am Coll Cardiol, 2001, 37.

7. K. T. Spencer, J. Bednarz, P. G. Rafter, C. Korcarz and R. M. Lang, Am J Cardiol, 1998, 82.

8. J. M. Tsutsui, R. R. Maciel, J. M. Costa, J. L. Andrade, J. F. Ramires and W. Mathias, Cardiovascular Ultrasound, 2004, 2, 1-5.

9. M. A. Perkins, Physics in Medicine and Biology, 1989, 34, 1645.

10. P. Vignon, M. B. Frank, J. Lesage, F. Mucke, B. Francois, S. Normand, M. Bonnivard, M. Clavel and H. Gastinne, Intensive Care Med, 2004, 30.

11. M. A. Quinones, P. S. Douglas, E. Foster, J. I. I. I. Gorcsan, J. F. Lewis, A. S. Pearlman, J. Rychik, E. E. Salcedo, J. B. Seward, J. G. Stevenson, D. M. Thys, H. H. Weitz and W. A. Zoghbi, J Am Coll Cardiol, 2003, 41. 
12. W. A. Zoghbi, M. Enriquez-Sarano, E. Foster, P. A. Grayburn, C. D. Kraft, R. A. Levine, P. Nihoyannopoulos, C. M. Otto, M. A. Quinones, H. Rakowski, W. J. Stewart, A. Waggoner and N. J. Weissman, J Am Soc Echocardiogr, 2003, 16.

13. M. A. Quinones, C. M. Otto, M. Stoddard, A. Waggoner and W. A. Zoghbi, J Am Soc Echocardiogr, 2002, 15.

14. S. N. Weingart and L. I. Iezzoni, JAMA, 2003, 290, 1917-1919.

15. Y. F. Tai and P. Piccini, Journal of Neurology, Neurosurgery \& Psychiatry, 2004, 75, 669-676.

16. K. Facey, I. Bradbury, G. Laking and L. Payne, Health Technology Assessment, 2007, 11, 288.

17. P. Ö. Kiratli, M. Tuncel and Z. Bar-Sever, Seminars in Nuclear Medicine, 2016, 46, 308-323.

18. R. Damadian, L. Minkoff, M. Goldsmith, M. Stanford and J. Koutcher, Science, 1976, 194, 1430-1432.

19. P. Mroz, J. T. Hashmi, Y.-Y. Huang, N. Lange and M. R. Hamblin, Expert Rev Clin Immunol, 2011, 7, 75-91.

20. P. Caravan, C. T. Farrar, L. Frullano and R. Uppal, Contrast Media \& Molecular Imaging, 2009, 4, 89-100.

21. J. A. Seibert, Health Physics, 1995, 69, 695-720.

22. R. Kuo, M. Panchal, L. Tanenbaum and J. V. Crues, Journal of Magnetic Resonance Imaging, 2007, 25, 245-261.

23. F. Bloch, W. W. Hansen and M. Packard, Physical Review, 1946, 69, 127-127. 
24. G. M. Bydder, J. V. Hajnal and I. R. Young, Clinical Radiology, 1998, 53, 159176.

25. K. R. Thulborn, J. C. Waterton, P. M. Matthews and G. K. Radda, Biochimica et Biophysica Acta (BBA) - General Subjects, 1982, 714, 265-270.

26. H. Seki, M. Kimura and K. Sakai, Clinical Radiology, 1997, 52, 18-23.

27. M. P. Lowe, Australian Journal of Chemistry, 2002, 55, 551-556.

28. S. Aime, A. S. Batsanov, M. Botta, J. A. K. Howard, D. Parker, K. Senanayake and G. Williams, Inorganic Chemistry, 1994, 33, 4696-4706.

29. J. H. Freed, The Journal of Chemical Physics, 1978, 68, 4034-4037.

30. D. Parker, R. S. Dickins, H. Puschmann, C. Crossland and J. A. K. Howard, Chemical Reviews, 2002, 102, 1977-2010.

31. S. Aime, M. Fasano and E. Terreno, Chemical Society Reviews, 1998, 27, 19-29.

32. Y. Okuhata, Advanced Drug Delivery Reviews, 1999, 37, 121-137.

33. P. Caravan, in Medicinal Inorganic Chemistry, American Chemical Society, 2005, vol. 903, pp. 166-191.

34. D. Artemov, Journal of Cellular Biochemistry, 2003, 90, 518-524.

35. E. J. Werner, A. Datta, C. J. Jocher and K. N. Raymond, Angewandte Chemie International Edition, 2008, 47, 8568-8580.

36. M. Rudin and R. Weissleder, Nat Rev Drug Discov, 2003, 2, 123-131.

37. B. Jebasingh and V. Alexander, Inorganic Chemistry, 2005, 44, 9434-9443.

38. L. G. V. Uitert and C. G. Haas, Journal of the American Chemical Society, 1953, 75, 451-455. 
39. W. P. Cacheris, S. K. Nickle and A. D. Sherry, Inorganic Chemistry, 1987, 26, 958-960.

40. P. Caravan, N. J. Cloutier, M. T. Greenfield, S. A. McDermid, S. U. Dunham, J. W. M. Bulte, J. C. Amedio, R. J. Looby, R. M. Supkowski, W. D. Horrocks, T. J. McMurry and R. B. Lauffer, Journal of the American Chemical Society, 2002, 124, 3152-3162.

41. A. D. Sherry and Y. Wu, Current opinion in chemical biology, 2013, 17, 167-174.

42. S. Laus, R. Ruloff, É. Tóth and A. E. Merbach, Chemistry - A European Journal, 2003, 9, 3555-3566.

43. R. B. Lauffer, Chemical Reviews, 1987, 87, 901-927.

44. in The Chemistry of Contrast Agents in Medical Magnetic Resonance Imaging, John Wiley \& Sons, Ltd, 2013, pp. i-xvi.

45. P. Caravan, Chemical Society Reviews, 2006, 35, 512-523.

46. F. Benetollo, G. Bombieri, L. Calabi, S. Aime and M. Botta, Inorganic Chemistry, 2003, 42, 148-157.

47. S. Avedano, L. Tei, A. Lombardi, G. B. Giovenzana, S. Aime, D. Longo and M. Botta, Chemical Communications, 2007, 4726-4728.

48. S. L. Fossheim, A. K. Fahlvik, J. Klaveness and R. N. Muller, Magnetic Resonance Imaging, 1999, 17, 83-89.

49. N. Kamaly and A. D. Miller, International Journal of Molecular Sciences, 2010.

50. E. Nakamura, K. Makino, T. Okano, T. Yamamoto and M. Yokoyama, Journal of Controlled Release, 2006, 114, 325-333. 
51. Y. Liu, Z. Chang, H. Yuan, A. M. Fales and T. Vo-Dinh, Nanoscale, 2013, 5, 12126-12131.

52. W.-I. Lin, C.-Y. Lin, Y.-S. Lin, S.-H. Wu, Y.-R. Huang, Y. Hung, C. Chang and C.-Y. Mou, Journal of Materials Chemistry B, 2013, 1, 639-645.

53. M. Ceulemans, K. Nuyts, W. De Borggraeve and T. Parac-Vogt, Inorganics, $2015,3,516$.

54. W. Yang, J. Feng and H. Zhang, Journal of Materials Chemistry, 2012, 22, 68196823.

55. P. Caravan, A. V. Astashkin and A. M. Raitsimring, Inorganic Chemistry, 2003, 42, 3972-3974.

56. A. V. Astashkin, A. M. Raitsimring and P. Caravan, The Journal of Physical Chemistry A, 2004, 108, 1990-2001.

57. S. Aime, S. G. Crich, E. Gianolio, G. Giovenzana, L. Tei and E. Terreno, Coordination Chemistry Reviews, 2006, 250, 1562-1579.

58. J. B. Livramento, É. Tóth, A. Sour, A. Borel, A. E. Merbach and R. Ruloff, Angewandte Chemie International Edition, 2005, 44, 1480-1484.

59. J. Yu, D. Javier, M. A. Yaseen, N. Nitin, R. Richards-Kortum, B. Anvari and M. S. Wong, Journal of the American Chemical Society, 2010, 132, 1929-1938.

60. A. A. Antipov and G. B. Sukhorukov, Advances in Colloid and Interface Science, 2004, 111, 49-61.

61. V. Yurgel, T. Collares and F. Seixas, Brazilian Journal of Medical and Biological Research, 2013, 46, 486-501. 
62. S. E. Plush, M. Woods, Y.-F. Zhou, S. B. Kadali, M. S. Wong and A. D. Sherry, Journal of the American Chemical Society, 2009, 131, 15918-15923.

63. I. L. Radtchenko, G. B. Sukhorukov, S. Leporatti, G. B. Khomutov, E. Donath and H. Möhwald, Journal of Colloid and Interface Science, 2000, 230, 272-280.

64. H. G. Bagaria and M. S. Wong, Journal of Materials Chemistry, 2011, 21, 94549466.

65. S. Zonghai, H. Dehong, X. Miaomiao, H. Meng, G. Ping and C. Lintao, NanoMicro Letters, 2013, 5, 145-150.

66. A. D. Sherry and M. Woods, Annual review of biomedical engineering, 2008, 10, $391-411$.

67. T. C. Soesbe, Y. Wu and A. D. Sherry, NMR in biomedicine, 2013, 26, 829-838.

68. K. M. Ward, A. H. Aletras and R. S. Balaban, Journal of Magnetic Resonance, 2000, 143, 79-87.

69. A. X. Li, F. Wojciechowski, M. Suchy, C. K. Jones, R. H. E. Hudson, R. S. Menon and R. Bartha, Magnetic Resonance in Medicine, 2008, 59, 374-381.

70. D. L. Longo, P. Z. Sun, L. Consolino, F. C. Michelotti, F. Uggeri and S. Aime, Journal of the American Chemical Society, 2014, 136, 14333-14336.

71. S. Zhang, K. Zhou, G. Huang, M. Takahashi, A. Dean Sherry and J. Gao, Chemical Communications, 2013, 49, 6418-6420.

72. V. R. Sheth, G. Liu, Y. Li and M. D. Pagel, Contrast Media \& Molecular Imaging, 2012, 7, 26-34.

73. C. Witte, V. Martos, H. M. Rose, S. Reinke, S. Klippel, L. Schröder and C. P. R. Hackenberger, Angewandte Chemie International Edition, 2015, 54, 2806-2810. 
REFERENCES

74. P. C. M. van Zijl, C. K. Jones, J. Ren, C. R. Malloy and A. D. Sherry, Proceedings of the National Academy of Sciences of the United States of America, 2007, 104, 4359-4364.

75. A. Tietze, J. Blicher, I. K. Mikkelsen, L. Østergaard, M. K. Strother, S. A. Smith and M. J. Donahue, NMR in biomedicine, 2014, 27, 163-174.

76. R. Trokowski, J. Ren, F. K. Kálmán and A. D. Sherry, Angewandte Chemie International Edition, 2005, 44, 6920-6923.

77. K. W. Y. Chan, T. Yu, Y. Qiao, Q. Liu, M. Yang, H. Patel, G. Liu, K. W. Kinzler, B. Vogelstein, J. W. M. Bulte, P. C. M. van Zijl, J. Hanes, S. Zhou and M. T. McMahon, Journal of Controlled Release, 2014, 180, 51-59.

78. J. Ren, R. Trokowski, S. Zhang, C. R. Malloy and A. D. Sherry, Magnetic Resonance in Medicine, 2008, 60, 1047-1055.

79. L. Q. Chen and M. D. Pagel, Advances in Radiology, 2015, 2015, 25.

80. S. Sinharay and M. D. Pagel, Annual Review of Analytical Chemistry, 2016, 9, 95115.

81. K. Hoshino, H. Q. Ly, J. V. Frangioni and R. J. Hajjar, Progress in Cardiovascular Diseases, 2007, 49, 414-420.

82. S. Zhang, Z. Kovacs, S. Burgess, S. Aime, E. Terreno and A. D. Sherry, Chemistry - A European Journal, 2001, 7, 288-296.

83. T. K. Stevens, M. Milne, A. A. H. Elmehriki, M. Suchý, R. Bartha and R. H. E. Hudson, Contrast Media \& Molecular Imaging, 2013, 8, 289-292.

84. K. J. Miller, A. A. Saherwala, B. C. Webber, Y. Wu, A. D. Sherry and M. Woods, Inorganic Chemistry, 2010, 49, 8662-8664. 
85. H.-Y. Hu, N.-H. Lim, D. Ding-Pfennigdorff, J. Saas, K. U. Wendt, O. Ritzeler, H. Nagase, O. Plettenburg, C. Schultz and M. Nazare, Bioconjugate Chemistry, 2015, 26, 383-388.

86. C. E. Carney, A. D. Tran, J. Wang, M. C. Schabel, A. D. Sherry and M. Woods, Chemistry (Weinheim an der Bergstrasse, Germany), 2011, 17, 10372-10378.

87. J. A. Pikkemaat, R. T. Wegh, R. Lamerichs, R. A. van de Molengraaf, S. Langereis, D. Burdinski, A. Y. F. Raymond, H. M. Janssen, B. F. M. de Waal, N. P. Willard, E. W. Meijer and H. Grüll, Contrast Media \& Molecular Imaging, 2007, 2, 229-239.

88. K. M. Payne, E. J. Valente, S. Aime, M. Botta and M. Woods, Chemical communications (Cambridge, England), 2013, 49, 2320-2322.

89. S. Zhang, K. Wu and A. D. Sherry, Journal of the American Chemical Society, 2002, 124, 4226-4227.

90. D. Pubanz, G. Gonzalez, D. H. Powell and A. E. Merbach, Inorganic Chemistry, 1995, 34, 4447-4453.

91. S. Aime, M. Botta, M. Fasano, S. Paoletti, P. L. Anelli, F. Uggeri and M. Virtuani, Inorganic Chemistry, 1994, 33, 4707-4711.

92. T. Mani, G. Tircso, O. Togao, P. Zhao, T. Soesbe, M. Takahashi and A. D. Sherry, Contrast Media \& Molecular Imaging, 2009, 4, 183-191.

93. B. N. Siriwardena-Mahanama and M. J. Allen, Molecules (Basel, Switzerland), 2013, 18, 9352-9381.

94. W. S. Fernando, A. F. Martins, P. Zhao, Y. Wu, G. E. Kiefer, C. Platas-Iglesias and A. D. Sherry, Inorganic Chemistry, 2016, 55, 3007-3014. 
95. P. M. Winter, Scientifica, 2014, 2014, 746574.

96. G. M. Lanza, P. M. Winter, S. D. Caruthers, A. M. Morawski, A. H. Schmieder, K. C. Crowder and S. A. Wickline, Journal of Nuclear Cardiology, 2004, 11, 733-743.

97. S. H. Koenig and W. E. Schillinger, Journal of Biological Chemistry, 1969, 244, 3283-3289.

98. P. C. Lauterbur, Nature, 1973, 242, 190-191.

99. S. Laurent, L. V. Elst and R. N. Muller, Contrast Media \& Molecular Imaging, 2006, 1, 128-137.

100. P. Reimer, G. Schneider and W. Schima, European Radiology, 2004, 14, 559-578.

101. Z. Zhou and Z.-R. Lu, Wiley interdisciplinary reviews. Nanomedicine and nanobiotechnology, 2013, 5, 1-18.

102. M. A. Yaseen, J. Yu, B. Jung, M. S. Wong and B. Anvari, Molecular Pharmaceutics, 2009, 6, 1321-1332.

103. S. B. Kadali, N. Soultanidis and M. S. Wong, Topics in Catalysis, 2008, 49, 251258.

104. Y. Huang, P. G. Lawrence and Y. Lapitsky, Langmuir, 2014, 30, 7771-7777.

105. N. Raghunand, C. Howison, A. D. Sherry, S. Zhang and R. J. Gillies, Magnetic Resonance in Medicine, 2003, 49, 249-257.

106. X. Wu, A. C. Dawsey, B. N. Siriwardena-Mahanama, M. J. Allen and T. J. Williams, Journal of Fluorine Chemistry, 2014, 168, 177-183.

107. M. Botta, European Journal of Inorganic Chemistry, 2000, 2000, 399-407. 
REFERENCES

108. E. Zitha-Bovens, R. N. Muller, S. Laurent, L. Vander Elst, C. F. G. C. Geraldes, H. van Bekkum and J. A. Peters, Helvetica Chimica Acta, 2005, 88, 618-632.

109. Z. Kotková, G. A. Pereira, K. Djanashvili, J. Kotek, J. Rudovský, P. Hermann, L. Vander Elst, R. N. Muller, C. F. G. C. Geraldes, I. Lukeš and J. A. Peters, European Journal of Inorganic Chemistry, 2009, 2009, 119-136.

110. G. A. Pereira, L. Ball, A. D. Sherry, J. A. Peters and C. F. G. C. Geraldes, Helvetica Chimica Acta, 2009, 92, 2532-2551.

111. S. Aime, D. Delli Castelli and E. Terreno, Angewandte Chemie International Edition, 2005, 44, 5513-5515.

112. L. Lattuada, A. Barge, G. Cravotto, G. B. Giovenzana and L. Tei, Chemical Society Reviews, 2011, 40, 3019-3049.

113. W. Zhang, J. A. Peters, F. Mayer, L. Helm and K. Djanashvili, The Journal of Physical Chemistry C, 2015, 119, 5080-5089.

114. V. S. Murthy, R. K. Rana and M. S. Wong, The Journal of Physical Chemistry B, 2006, 110, 25619-25627.

115. A. Farashishiko, K. N. Chacón, N. J. Blackburn and M. Woods, Contrast Media \& Molecular Imaging, 2015, n/a-n/a.

116. J. S. Kłos and J. U. Sommer, Macromolecules, 2009, 42, 4878-4886.

117. M. S. Wong, J. N. Cha, K.-S. Choi, T. J. Deming and G. D. Stucky, Nano Letters, 2002, 2, 583-587.

118. S. Aime, M. Botta, Z. Garda, B. E. Kucera, G. Tircso, V. G. Young and M. Woods, Inorganic Chemistry, 2011, 50, 7955-7965.

119. P. Caravan, Accounts of Chemical Research, 2009, 42, 851-862. 
REFERENCES

120. T. E. McCann, N. Kosaka, P. L. Choyke and H. Kobayashi, in In Vivo Cellular Imaging Using Fluorescent Proteins: Methods and Protocols, ed. M. R. Hoffman, Humana Press, Totowa, NJ, 2012, pp. 191-204.

121. S. Dumas, V. Jacques, W.-C. Sun, J. S. Troughton, J. T. Welch, J. M. Chasse, H. Schmitt-Willich and P. Caravan, Investigative radiology, 2010, 45, 600-612.

122. V. Jacques, S. Dumas, W.-C. Sun, J. S. Troughton, M. T. Greenfield and P. Caravan, Investigative radiology, 2010, 45, 613-624.

123. G. Liu, Case Western Reserve University, 2008.

124. M. Milne, M. Lewis, N. McVicar, M. Suchy, R. Bartha and R. H. E. Hudson, RSC Advances, 2014, 4, 1666-1674.

125. N. McVicar, A. X. Li, M. Suchý, R. H. E. Hudson, R. S. Menon and R. Bartha, Magnetic Resonance in Medicine, 2013, 70, 1016-1025.

126. W. ThomasDixon, J. Ren, A. J. M. Lubag, J. Ratnakar, E. Vinogradov, I. Hancu, R. E. Lenkinski and A. D. Sherry, Magnetic resonance in medicine : official journal of the Society of Magnetic Resonance in Medicine / Society of Magnetic Resonance in Medicine, 2010, 63, 625-632.

127. B. Yoo, M. S. Raam, R. M. Rosenblum and M. D. Pagel, Contrast Media \& Molecular Imaging, 2007, 2, 189-198.

128. M. M. Ali, G. Liu, T. Shah, C. A. Flask and M. D. Pagel, Accounts of Chemical Research, 2009, 42, 915-924.

129. C.-H. Huang and J. R. Morrow, Journal of the American Chemical Society, 2009, 131, 4206-4207. 
REFERENCES

130. Y. Li, V. R. Sheth, G. Liu and M. D. Pagel, Contrast Media \& Molecular Imaging, 2011, 6, 219-228.

131. L. M. De Leon-Rodriguez, A. J. M. Lubag, C. R. Malloy, G. V. Martinez, R. J. Gillies and A. D. Sherry, Accounts of Chemical Research, 2009, 42, 948-957.

132. S. J. Dorazio, A. O. Olatunde, P. B. Tsitovich and J. R. Morrow, JBIC Journal of Biological Inorganic Chemistry, 2014, 19, 191-205.

133. S. Zhang, M. Merritt, D. E. Woessner, R. E. Lenkinski and A. D. Sherry, Accounts of Chemical Research, 2003, 36, 783-790.

134. N. Goffeney, J. W. M. Bulte, J. Duyn, L. H. Bryant and P. C. M. van Zijl, Journal of the American Chemical Society, 2001, 123, 8628-8629.

135. S. Aime, D. Delli Castelli and E. Terreno, Angewandte Chemie International Edition, 2003, 42, 4527-4529.

136. Y. Wu, Y. Zhou, O. Ouari, M. Woods, P. Zhao, T. C. Soesbe, G. E. Kiefer and A. D. Sherry, Journal of the American Chemical Society, 2008, 130, 13854-13855.

137. I. Hancu, W. T. Dixon, M. Woods, E. Vinogradov, A. D. Sherry and R. E. Lenkinski, Acta Radiologica, 2010, 51, 910-923.

138. C. Oerlemans, W. Bult, M. Bos, G. Storm, J. F. W. Nijsen and W. E. Hennink, Pharmaceutical Research, 2010, 27, 2569-2589.

139. P. M. Winter, S. D. Caruthers, X. Yu, S. K. Song, J. Chen, B. Miller, J. W. Bulte, J. D. Robertson, P. J. Gaffney, S. A. Wickline and G. M. Lanza, Magn Reson Med, 2003, 50, 411-416.

140. S. Aime, C. Carrera, D. Delli Castelli, S. Geninatti Crich and E. Terreno, Angewandte Chemie International Edition, 2005, 44, 1813-1815. 